\title{
Delay-Power-Rate-Distortion Model for Wireless Video Communication Under Delay and Energy Constraints
}

\author{
Chenglin Li, Student Member, IEEE, Dapeng Wu, Fellow, IEEE, Hongkai Xiong, Senior Member, IEEE
}

\begin{abstract}
Smart mobile phones are capable of performing video coding and streaming over wireless networks, but are often constrained by the end-to-end delay requirement and energy supply. To achieve optimal performance under the delay and energy constraints, in this paper, we extend the traditional R-D model and the previously proposed d-R-D model to a novel delay-power-rate-distortion (d-P-R-D) model by including another two dimensions (the encoding time and encoder power consumption), which quantifies the relationship among source encoding delay, rate, distortion and power consumption for IPPPP coding mode in H.264/AVC. We have verified the accuracy of our proposed d-P-R-D model through experiments. Based on the proposed d-P-R-D model, we develop a novel rate control algorithm, which minimizes the encoding distortion under the constraints of rate, delay and power. Experimental results demonstrate the superiority of the proposed rate control algorithm over the existing scheme. Therefore, the d-P-R-D model and the model based rate control provide a theoretical basis and a practical guideline for the cross-layer system design and performance optimization in wireless video communication under delay and energy constraints.
\end{abstract}

\section{Index Terms}

Delay-power-rate-distortion model, wireless video, H.264/AVC, video coding, rate control.

\section{INTRODUCTION}

Wireless video communication systems, including both video encoding and streaming over wireless communication networks, have experienced extensive growth in the last decades and been utilized for a wide range of applications, such as video surveillance, emergency response, consumer electronics multimedia systems, and mobile video services [1]. For example, real-time

This work was supported in part by National Science Foundation under grant ECCS-1002214, CNS-1116970, the Joint Research Fund for Overseas Chinese Young Scholars under grant No. 61228101, and the National Natural Science Foundation of China, under grant No. 61221001.

C. Li and H. Xiong are with the Department of Electronic Engineering, Shanghai Jiao Tong University, Shanghai 200240, China (e-mail: lc11985@sjtu.edu.cn; xionghongkai@sjtu.edu.cn).

D. Wu is with the Department of Electrical and Computer Engineering, University of Florida, Gainesville, FL 32611 USA (e-mail: wu@ece.ufl.edu). 


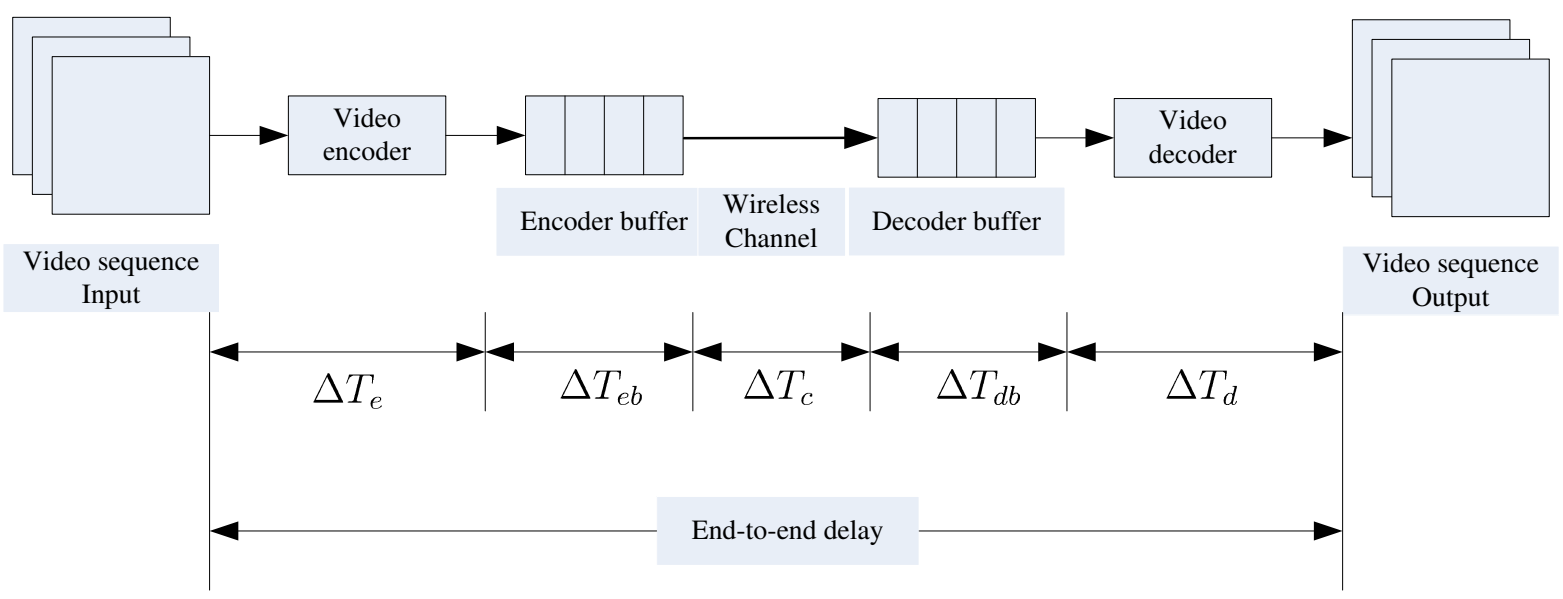

Fig. 1. End-to-end delay components of a video communication system.

entertainment traffic that comprises streaming both video and audio has already been making up the majority of Internet traffic [2]. According to the Cisco visual networking index, mobile video communication application will grow at a compound annual growth rate of 75 percent between 2012 and 2017, the highest growth rate of any mobile application category [3]. Such predictions lead to a natural but challenging question: how can we guarantee the quality of service (QoS) metrics, such as end-to-end distortion, and end-to-end delay, for the wireless video communication systems?

From the perspective of video encoding, if the video encoder is separately investigated without consideration of its relationship with the subsequent transmission and the whole wireless video communication system, transitional rate control (RC) plays an important role that affects the overall R-D performance in the hybrid video codec design [4]. Based on the rate-distortion optimization (RDO), rate control aims at minimizing the encoding distortion under a given constraint on the encoding rate, by appropriate selections of several coding parameters, such as quantization parameter $(Q P)$ and macroblock $(\mathrm{MB})$ mode. In this case, the encoding time and power consumption are of little concern to the video encoder, since it can be assumed that there is no limit on the encoding time and power consumption.

For the purpose of achieving the best end-to-end QoS performance, however, the entire cross-layer wireless video communication system is expected to appropriately assign for the video encoder both encoding time and encoding power according to the total end-to-end delay constraint and a given maximum power supply. More specifically, for a practical real-time 
wireless video communication system, the end-to-end delay can be broken up into several delay components which, as illustrated in Fig. 1 respectively, are video encoding delay $\Delta T_{e}$, encoder buffer delay $\Delta T_{e b}$, channel transmission delay $\Delta T_{c}$, decoder buffer delay $\Delta T_{d b}$, and video decoding delay $\Delta T_{d}$ [5], [6]. In [5] and [7], the video encoding time $\Delta T_{e}$ and decoding time $\Delta T_{d}$ are both assumed to be constant. In fact, the video decoding time can be considered as a part of the video encoding time, since the encoder has to decode the video sequence as well. As illustrated in [1], [8], however, the video encoding time (delay) is determined by the video encoding complexity, while the video encoding complexity would affect the source coding incurred distortion and bit rate. Consequently, both the distortion and bit rate of the compressed video that is transmitted over the channel is controlled by the video encoding time. On the other hand, given an end-to-end delay constraint, if the encoding time is increased to achieve better compression performance, the allowed queueing and buffering delay at encoding/decoding buffers and channel delay for transmission will decrease accordingly, which in turn decreases the delay constrained transmission throughput and thus increases the transmission distortion of the compressed video. Generally, for a given end-to-end delay constraint, the overall system performance depends on the allocation of end-to-end delay to different delay components. The change of delay assignment in one component would cause changes of the delay budget in other components, which would affect the overall system performance. Likewise, the mobile video communication system is also power limited and needs to allocate its power supply to the encoding/decoding modules and transmitting/receiving modules. For a given maximum power supply, the change of the encoding power consumption would also impact on the power budget in other modules and the overall system performance as well. Therefore, when the subsequent video transmission is taken into account, video encoding delay and video encoding power consumption become two new constraints which, as well, would affect the overall R-D performance of the video communication systems and need to be considered in the rate control of the video encoder.

From the perspective of the cross-layer design of a wireless video communication system, the delay and power consumption constraints on the video encoder are two-folded. On the one hand, efficient video compression results in reduction of the bit rate of the video data, leading to reduction in transmission power and/or transmission delay at the physical layer, or reduction in the transmission rate and transmission error rate at data link layer. On the other hand, efficient video compression often requires high computational complexity, leading to large 
encoder power consumption and long encoding delay at the application layer. As implied by these two conflicting aspects, there is a tradeoff among delay $d$, power consumption $P$, rate $R$, and video distortion $D$ for the design of the video encoder, which could be further applied to control the QoS performance of the entire cross-layer wireless video communication system [9]. To find the optimal tradeoff solution, we need to develop an analytic framework to model the delay-power-rate-distortion (d-P-R-D) behavior of the video encoder.

\section{A. Related Work}

Many rate control schemes have been proposed in literature to provide good video quality for the encoded video while keeping its output bit rate within the bandwidth constraint for video communication. Due to its efficiency, in the state-of-the-art video coding standard H.264/AVC, JVT-G012 [10] is adopted by simply tuning $Q P$ to meet the target bit rate. In [11], the interdependency between rate-distortion optimization and rate control is further improved by $Q P$ estimation and update. Considering the initial $Q P$ for the first I-frame would influence the rate control performance, a rate control scheme with adaptive $Q P$ initialization is proposed in a content-aware manner [12]. In order to achieve a relatively steady visual quality, authors in [13] develop a bit allocation scheme for both I-frame and P-frame based on the frame complexity measurement and estimation model. Obviously, these schemes only focus on the R-D performance of the video encoding system, while the other two dimensions, the encoding time and power consumption are not taken into account.

In order to formulate the rate-distortion optimization problem, several bit rate and quantization distortion models have been developed. Most existing work, e.g., [4] [14], derives the bit rate as a function of video statistics and the quantization step size (or quantization parameter $Q P$, there is a one-to-one mapping between the quantization step size $Q$ and the quantization parameter $Q P$ [15], with $Q$ increasing by $12.5 \%$ for each increment of 1 in $Q P$ ). Also, the quantization distortion is derived as a function of the quantization step size and video statistics for a uniform quantizer. In the rate-distortion model of [4], both the source rate and the source distortion for a hybrid video coder with block based coding are derived as functions of the standard deviation of the transformed residuals under the assumption of Laplacian distribution. By considering the characteristics of variances of transformed residuals and compensating the mismatch between the real histogram and the assumed Laplacian distribution, [16] improved both the bit rate 
and distortion model where the Lagrangian based RDO is solved by the bi-section search. To achieve the optimal selection of coding parameters, [4] converted the RDO to a Lagrangian optimization problem and derived an accurate function between the single Lagrange multiplier and quantization step size. However, none of them considers the analytic model of the encoding time and power consumption, which makes the RDO not appropriate for the situation when either encoding time or encoding power is constrained. Moreover, the bi-section search solver would result in a relatively high computational complexity.

Many works have been done to analyze the rate-distortion-complexity of video encoders [1], [6], [8], [17], [18], [19]. To derive the power-rate-distortion model for the video encoding system, He et al. [1] summarized the encoding complexity of H.263 video encoder as three modules: motion estimation, PRE-coding (transform, quantization, inverse quantization and inverse transform), and entropy coding. The relationship among the encoding complexity, rate, and distortion was analyzed, and the power consumption level was adopted to represent the encoding complexity. Unfortunately, this P-R-D model is dedicated to only H.263 video encoder. The model should be evolved since H.264/AVC utilizes the tree structured motion compensation with seven intermodes, which causes the motion estimation consumes much more encoding complexity than the other two modules. As a matter of fact, [1] also fails to consider the dimension of the encoding time which is relevant to the encoding complexity. To tackle these issues, the delayrate-distortion (d-R-D) model of H.264/AVC video encoders was proposed and analyzed in [6], [8] for both IPPPP and IBPBP coding modes. This d-R-D model depends on the quantization step size and the standard deviation of transformed residuals in motion estimation (ME), which was further fitted as functions of coding parameters in ME. However, this model did not consider and analyze the encoding power consumption that is also closely related to the encoding complexity. In addition, it neglects the critical impact of the quantization step size on both the standard deviation of transformed residuals and the encoding delay component, which is demonstrated based on extensive experiments.

\section{B. Proposed Research}

To our best knowledge, there has been no analytic framework for the d-P-R-D modeling of the video encoding system, which is of great importance to analyze the effect of the video encoding time and power consumption on the R-D performance of the video encoder. In this 
work, we extend from the traditional R-D model [4], [16] and the d-R-D model previously proposed in [6], [8], and accordingly develop an analytic framework to model, control and optimize the delay-power-rate-distortion (d-P-R-D) behavior of the H.264/AVC video encoding system. More specifically, our contributions in this paper are two-fold. First, four dimensions (rate, distortion, delay and power) that jointly determine the performance of the H.264/AVC video encoder are derived as functions of coding parameters (search range and number of reference frames in motion estimation, and quantization step size), respectively. Here, without loss of generality, the coding structure of the H.264/AVC encoder is chosen to be IPPPP coding mode, which is also reasonable since as will be introduced, the motion estimation module for inter-coded P-frames takes the major part of the entire encoding complexity. The model accuracy has also been validated and compensated by considering the statistics of both the current frame and the previous frame. Second, the proposed d-P-R-D model is applied to formulate the source rate control problem as a d-P-R-D optimization problem with respect to the search range and quantization step size. Compared with the existing work on source rate control aiming at minimizing the video encoding distortion, we have introduced two more constraints corresponding to the encoding time and the encoding power, in addition to the traditional rate constraint. Furthermore, a practical algorithm based on both Karush-Kuhn-Tucker (KKT) conditions and sequential quadratic programming (SQP) methods for the d-P-R-D optimization based rate control problem is developed, which can produce both primal (search range and quantization step size) and dual (Lagrange multipliers) solutions simultaneously and efficiently in an iterative way. The proposed d-P-R-D model and model based rate control algorithm provide a theoretical basis, as well as a practical guideline, for the cross-layer system design and performance optimization in wireless video communication under delay and energy constraints. By using the proposed d-P-R-D model, we can optimize the cross-layer performance (e.g., end-to-end distortion) by appropriately allocating the delay and power budget to components within the wireless video communication system. It should be noted that, besides delay and power limitation, bandwidth fluctuations and higher packet losses are also the challenges to be addressed in wireless video communication systems. The main scope of this paper is how to derive the d-P-R-D model and model based rate control problem for video encoders and propose the model based source rate control algorithm. Therefore, at the encoder side, we only consider the encoding delay which is a part of end-to-end delay, and encoding power which is a part 
of the total system power consumption. The bandwidth fluctuations and high packet losses are more related to the video packet transmission module of the wireless communication system, and would be taken into account in our sequel paper that investigates the d-P-R-D performance of the end-to-end wireless video communication systems comprising both video encoder and video packet transmission part.

\section{Paper Organization}

The rest of the paper is organized as follows. In Section II, we derive the d-P-R-D source coding model for H.264/AVC and verify the model accuracy based on experiments. In Section III, we formulate a d-P-R-D optimization based source rate control problem, and accordingly develop a practical algorithm based on KKT conditions and SQP methods to determine the optimal selection of coding parameters. Section IV presents the experimental results, which demonstrates the accuracy of the d-P-R-D model, the convergence behavior and performance of the proposed algorithm. The concluding remarks and the future work are given in Section V.

\section{D-P-R-D SOURCE CODING MOdeL}

According to the rate-distortion model proposed by Li [4] and Chen [16], both source rate and source distortion for a hybrid video coder with block based coding, e.g., H.264/AVC encoder, are based on the distribution of transformed residuals which is mainly determined by the motion estimation (ME) accuracy and quantization distortion. More specifically, under the assumption that the transformed residuals in ME follow Laplacian distribution [4] [20], the source rate and distortion of an inter-coded P-frame in IPPPP coding mode can be derived as functions of the standard deviation $\sigma$ of the transformed residuals and the quantization step size $Q$ (or quantization parameter $Q P$ ). The extension to other distributions (e.g., Generalized Gaussian distribution [21] and Cauchy distribution [22]) is also straightforward [16], since the transform coefficients are supposed to be independent and identically distributed (i.i.d.).

To further analyze ME accuracy in H.264/AVC [23], the standard deviation $\sigma$ of transformed residuals depends on the following four coding parameters: 1) macroblock (MB) coding mode, 2) ME search range $\lambda, 3$ ) the number of reference frames $\theta$ [6], [8], and 4) quantization step size $Q$. If the function relationship of $\sigma(\lambda, \theta, Q)$ can be established for H.264/AVC encoder, the 
source rate and distortion will become functions of ME parameters $\lambda, \theta$ and quantization step size $Q$.

On the other hand, both encoding time and encoding power are monotonously increasing with encoding complexity. As will be justified in Sec. II-B, ME module is the most complexity exhausting part within the entire encoding process and thus it is reasonable to approximate the entire encoding complexity by ME complexity, which is measured by the number of sum of absolute difference (SAD) operations for each MB partition (or subpartition): $\# S A D=(2 \lambda+$ $1)^{2} \times \theta$ [6], [8]. Therefore, by translating the specific coding behavior into encoding complexity, both encoding time and power can be expressed as functions of ME parameters $\lambda$ and $\theta$. If the encoding time is equivalently considered as video encoding delay, then the entire d-P-R-D source coding model can be formulated.

Next, the closed form functions of rate, distortion, delay and power will be derived, respectively.

\section{A. Source Rate and Distortion Model}

In order to develop the source rate and distortion model, the closed form function of $\sigma(\lambda, \theta, Q)$ would be generated first. Due to the lack of any prior knowledge of the exact function form, a basic means is to draw the relationship of $\sigma$ versus $\lambda, \theta$, and $Q$ which can be fitted by a known function form. To achieve it, the JM18.2 [24] coding engine is tested with the IPPPP coding mode where the Bus (QCIF, $176 \times 144)$, Foreman (CIF, $352 \times 288)$, and Mobile (CIF, $352 \times 288$ ) video sequences are used to collect the statistics with a wide range of scene activity pattern, including camera movement and large object motion (Bus), medium but complex motion (Foreman) and motion with zooming effects (Mobile). For a fair evaluation, all MBs in the experiments would select the same coding mode from the eight inter- modes except skip mode in order to exclude the potential influence of MB coding mode in ME. Accordingly, these inter- modes are indicated by index 1 to 7 as in JM configuration, (i.e., assigning index 1 to $16 \times 16$ inter- mode, index 2 to $16 \times 8$ inter- mode, etc.)

Since $\lambda, \theta$ and $Q$ are independently tuned parameters in JM 18.2 configurations, we separately evaluate their impacts on the average standard deviation $\sigma$ of transformed residuals. For all the seven inter- modes and the real mode selection where each MB chooses the best inter- mode based on RDO, respectively, Fig. 2 illustrates the relationship between average standard deviation 
$\sigma$ of transformed residuals and search range $\lambda$, with fixed $\theta$ and $Q$. It can be seen from Figs. 2(a) and 2(c) that every curve obtained under one of the seven inter- modes or the real mode selection resembles an exponential function with a constant vertical translation. In other words, all the curves of seven inter- modes and the real mode selection have the similar functional forms. In addition, as mode index increases from 1 to 7 , the MB partition (or sub-partition) size used to find the best match becomes smaller, which will lead to smaller predictive residuals and thus smaller $\sigma$ under the same search range $\lambda$. Accordingly, as presented in Fig. 2, the $\sigma$ vs. $\lambda$ curve would slightly drop along $\sigma$ direction as mode index increases. The curve of the real mode selection is located in the middle of these curves, because each MB should select a specific inter- mode out of the seven inter- modes. Comparing Figs. 2(a) and 2(c), it can also be observed that more $16 \times 16$ inter- modes are assigned to Foreman sequence than Bus sequence under the real mode selection. It can be indicated by the relative distance between the curve of the real mode selection and the curve of the $16 \times 16$ inter- mode. Based on the analysis, similarly as in [6], [8], we can use an exponential function with a constant vertical translation to fit the curves in Figs. 2(a) and 2(c),

$$
\sigma(\lambda)=a_{1} e^{-b_{1} \lambda}+c_{1}
$$

where $a_{1}, b_{1}$ and $c_{1}$ are fitting parameters. The corresponding fitting results are shown in Figs. 2(b) and 2(d).

It should be noted that in the training process of the source rate and distortion models, the fast search algorithm is used in motion estimation to investigate the relationship between $\sigma$ and $\lambda, \theta, Q$, for the sake of saving computational complexity. The peaks for certain $\lambda$ values in Figs. 2(a) and 2(c) are mainly because of the fast search algorithm. Specifically, with fast search algorithm, the optimal motion vector that minimizes the SAD for each macroblock may not be found, which would cause larger predictive residuals and thus larger $\sigma$ than the optimal values achieved by the exhaustive full search algorithm for certain $\lambda$ values.

Likewise, the impact of number of reference frames $\theta$ on the average standard deviation $\sigma$ of transformed residuals is investigated in Fig. 3, at fixed $\lambda$ and $Q$. The similar analysis and observations can be drawn for the $\sigma$ vs. $\theta$ curves as shown in Figs. 3(a) and 3(c). Hence, the $\sigma$ 


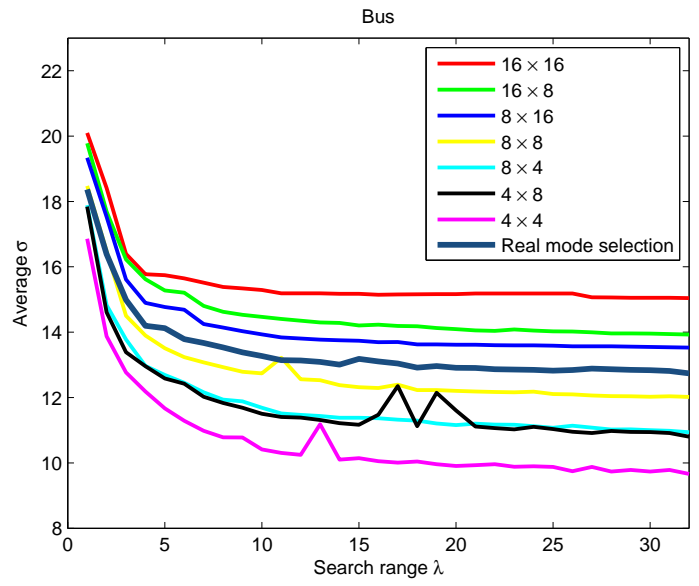

(a)

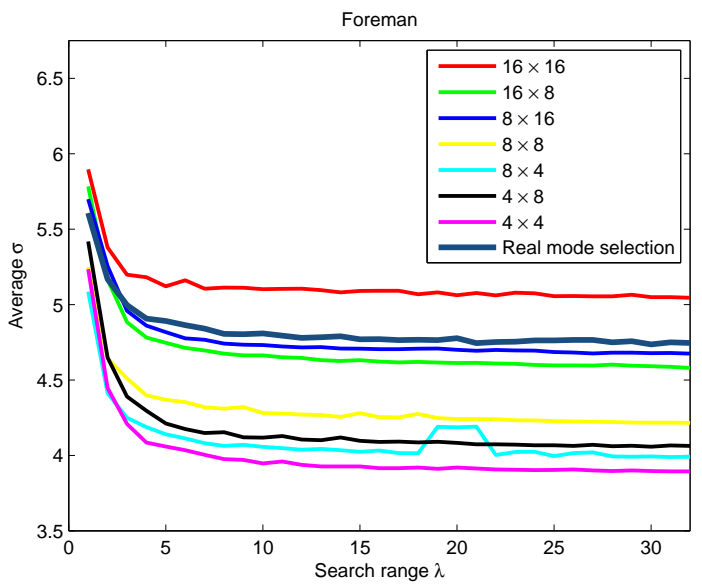

(c)

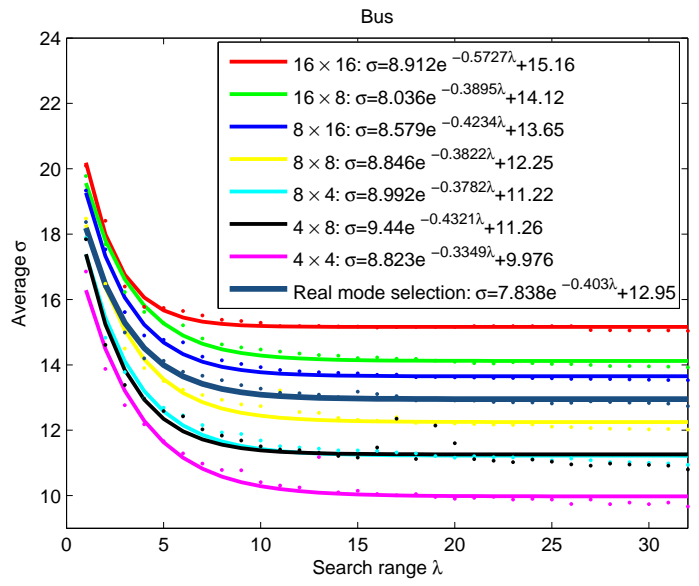

(b)

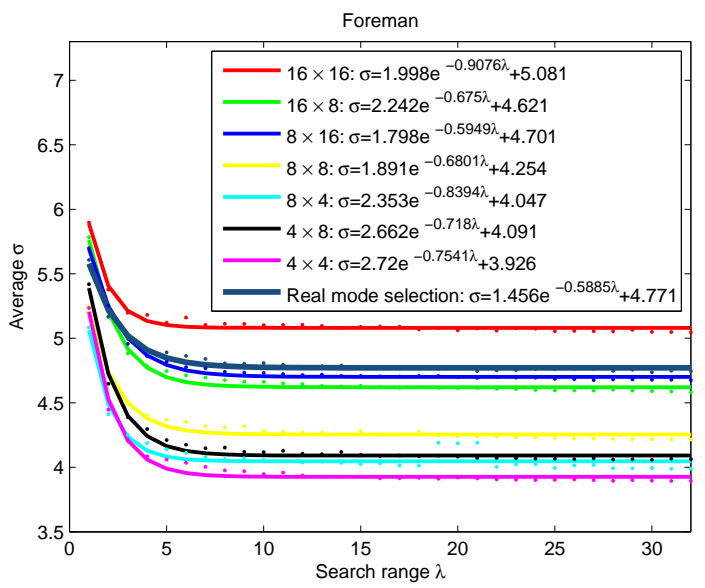

(d)

Fig. 2. Impact of search range on the average standard deviation of transformed residuals when $\theta=4$ and $Q=10$, (a) Bus video sequence, (b) the exponential fitting, and (c) Foreman video sequence, (d) the exponential fitting.

vs. $\theta$ curves can also be fitted by an exponential function plus a constant,

$$
\sigma(\theta)=a_{2} e^{-b_{2} \theta}+c_{2}
$$

where $a_{2}, b_{2}$ and $c_{2}$ are fitting parameters. The corresponding fitting results are shown in Figs. 3(b) and 3(d). In comparison to the fitting results in Figs. 2(b) and 2(d), it can be seen that since $\sigma$ vs. $\theta$ curves are much more flatter than $\sigma$ vs. $\lambda$ curves, the decreasing rate of fitted exponential functions in Fig. 3 is much smaller than that in Fig. 2. Therefore, search range $\lambda$ is a more effective parameter on the change of $\sigma$ than the number of reference frames $\theta$.

When $\lambda$ and $\theta$ are fixed, the relationship between average standard deviation $\sigma$ of transformed 


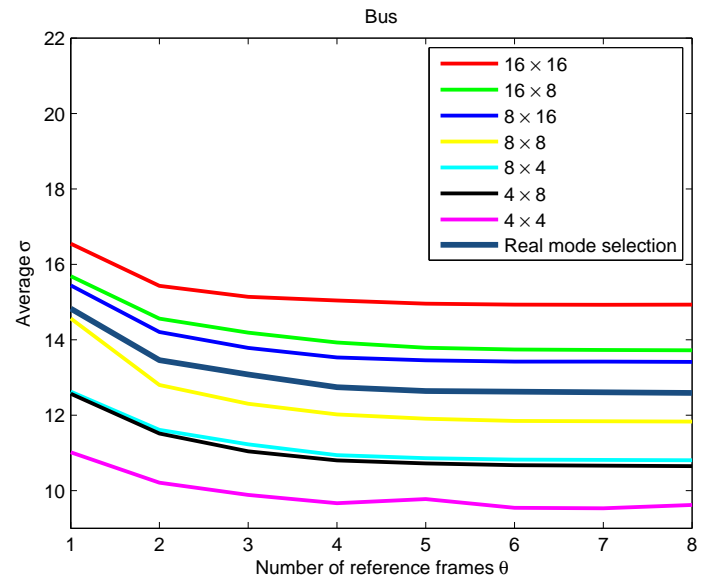

(a)

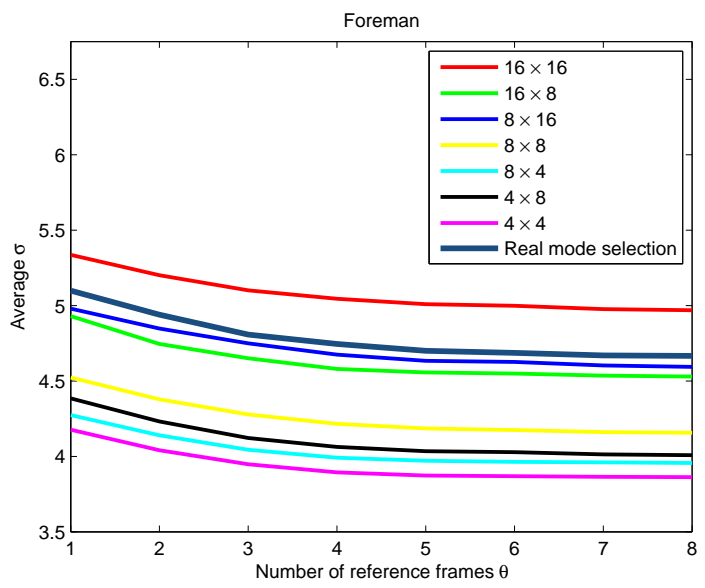

(c)

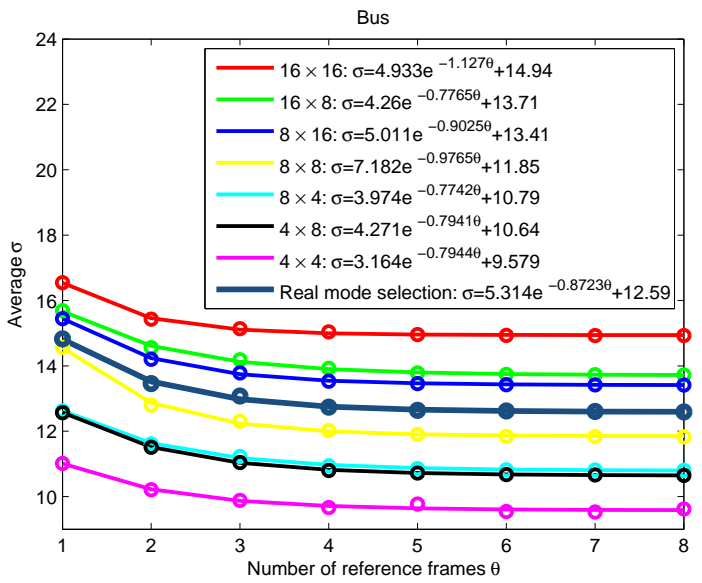

(b)

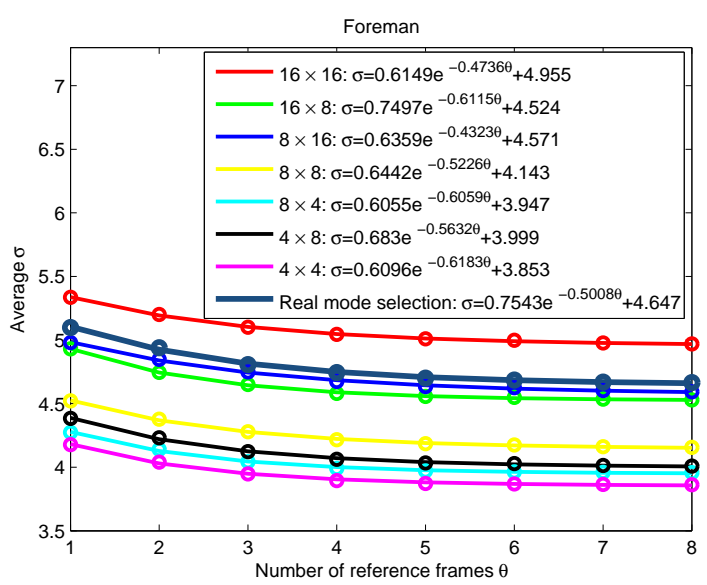

(d)

Fig. 3. Impact of number of reference frames on the average standard deviation of transformed residuals when $\lambda=32$ and $Q=10$, (a) Bus video sequence, (b) the exponential fitting, and (c) Foreman video sequence, (d) the exponential fitting.

residuals and quantization step size $Q$ is illustrated in Fig. 4. The $\sigma$ vs. $Q$ curves in Figs. 4(a) and 4(c) indicate that they can be simply fitted by a linear function,

$$
\sigma(Q)=a_{3} Q+b_{3}
$$

where $a_{3}$ and $b_{3}$ are fitting parameters. The corresponding linear fitting results are shown in Figs. 4(b) and 4(d). Similarly, quantization step size $Q$ is a more effective parameter on the change of $\sigma$ than the number of reference frames $\theta$.

In order to have a better understanding of Eqs. (1) - (3), we will discuss the impact of the aforementioned four different factors on $\sigma$. Generally, an inter- mode with higher mode index 


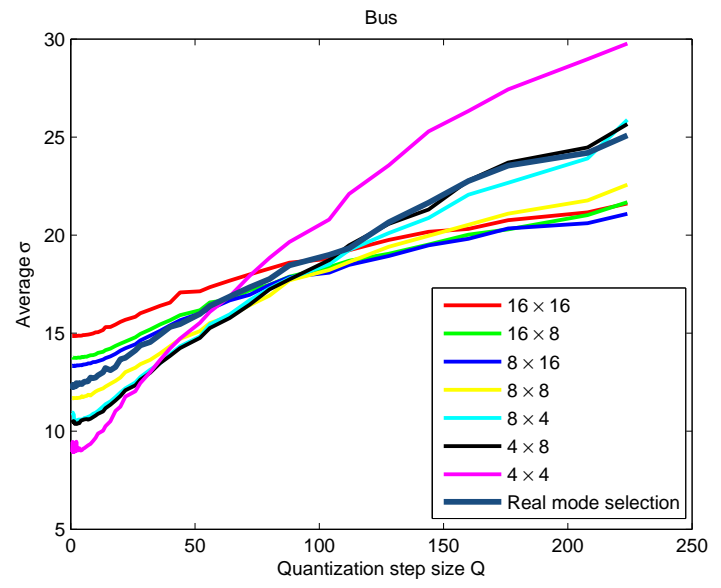

(a)

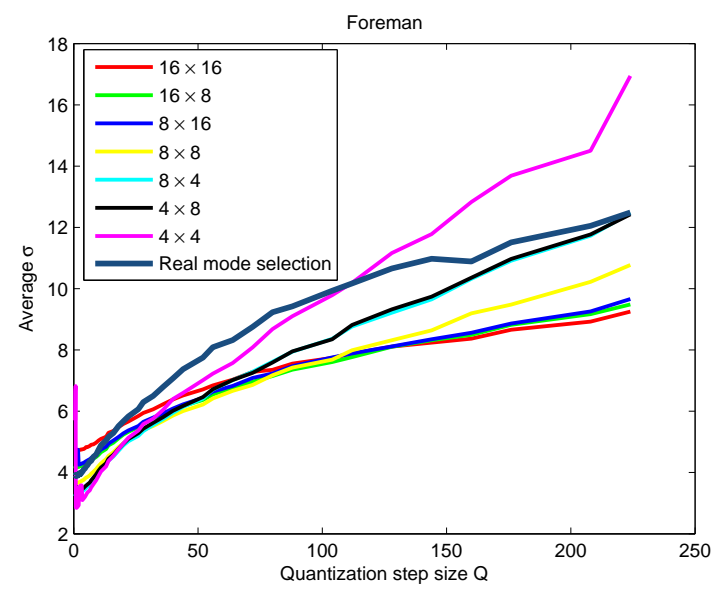

(c)

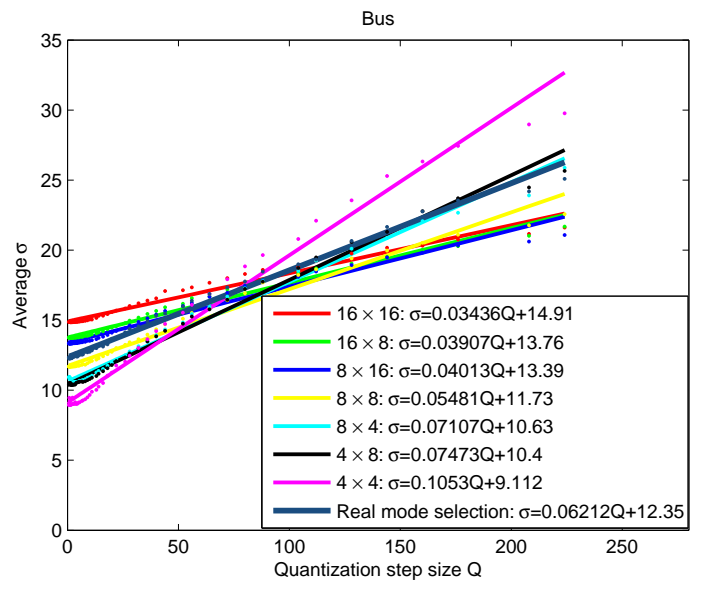

(b)

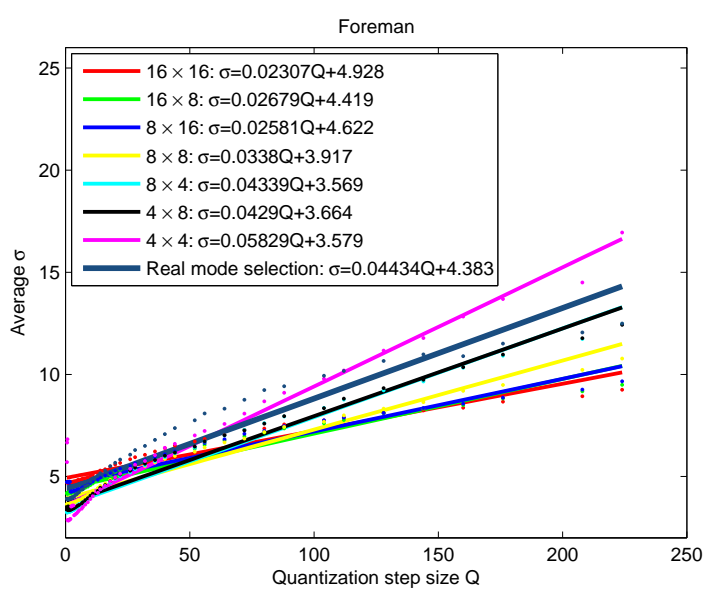

(d)

Fig. 4. Impact of quantization step size on the average standard deviation of transformed residuals when $\lambda=32$ and $\theta=4$, (a) Bus video sequence,(b) the linear fitting, and (c) Foreman video sequence, (d) the linear fitting.

(i.e., with smaller size of MB partitions) will lead to better prediction, and thus the standard deviation $\sigma$ of transformed residuals tends to be smaller. For the real mode selection, since MB can choose a best mode out of all the seven inter- modes, the value of $\sigma$ is bounded within mode 1 and mode 7. On the other hand, either a larger search range $\lambda$ or a larger number of reference frames $\theta$ will result in a bigger 3-D search cube in ME and thus a better prediction, which would also lead to a smaller $\sigma$. The last factor, quantization step size Q, would affect the distortion of the reference frames. Generally, the distortion of the reference frames will be increased by the selection of larger $Q$, which tends to result in larger $\sigma$.

From Figs. 2-4, it can also be seen that $\theta$ has little contribution to the change of $\sigma$ compared 


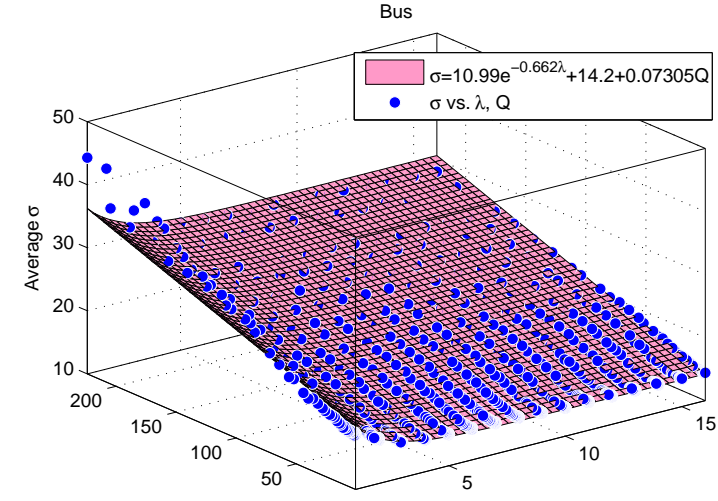

Quantization step size Q

Search range $\lambda$

(a)

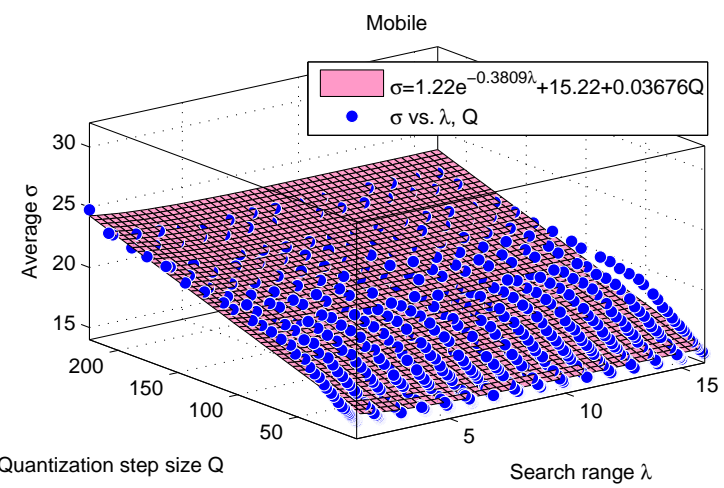

(c)

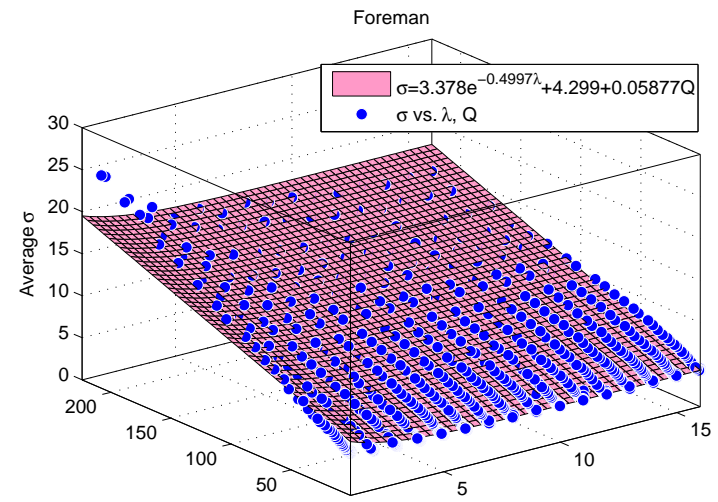

Quantization step size Q

Search range $\lambda$

(b)

Fig. 5. Two dimensional fitting of the average standard deviation of transformed residuals vs. search range and quantization step size, (a) Bus video sequence with $\theta=1$, R-square $=0.965$ and RMSE $=0.845$ (b) Foreman video sequence with $\theta=1$, R-square $=0.936$ and RMSE=0.884, (c) Mobile video sequence with $\theta=1$, R-square $=0.884$ and RMSE $=0.763$.

with the other two parameters. For example, for the same mode, the changing rate of $\sigma$ vs. $\theta$ is much smaller than that of $\sigma$ vs. $\lambda$ and $\sigma$ vs. $Q$. In the real mode selection, we could investigate the function $\sigma(\lambda, Q)$ for given $\theta=\theta_{0}$ which approximates the function $\sigma(\lambda, \theta, Q)$, for the sake of simplicity. Another motivation is that by fixing the value of $\theta$, the computational complexity for fitting the standard deviation function $\sigma(\lambda, \theta, Q)$ would be decreased. Fig. 5 illustrates the two dimensional fitting results of function $\sigma(\lambda, Q)$ with $\theta$ fixed at 1 . Considering both the exponential relationship with $\lambda$ and linear relationship with $Q$, the fitted two dimensional function of $\sigma$ can be represented in the form of

$$
\sigma(\lambda, Q)=a e^{-b \lambda}+c+d Q \approx \sigma(\lambda, \theta, Q)
$$


where $a, b, c$ and $d$ are fitting parameters. Based on the fitting results, we have obtained the closed form function of $\sigma(\lambda, Q)$, which is an approximation of $\sigma(\lambda, \theta, Q)$. To better assess the fitting performance, both R-square and RMSE (root mean square error) are used as the metrics to measure the degree of data variation from the proposed model (4), as shown in Fig. 5. It should be noted that although the fitting accuracy could be improved by using other high-order or complex function forms, the computational complexity would also be increased. In accordance with wireless video communication systems, and for the sake of simplicity, we only apply the simple function form as expressed by Eq. (4) which could also achieve overall acceptable fitting performance.

For i.i.d. zero-mean Laplacian source under the uniform quantizer, the closed form functions of entropy of quantized transform coefficients and quantization distortion have been provided in [16]. Furthermore, the entropy of quantized transformed residual can approximate the source rate model when no effect by side information such as macroblock type and motion information [4]. Hence, the closed form of source rate model is given by:

$$
R(\Lambda, Q)=H(\Lambda, Q)=-P_{0} \log _{2} P_{0}+\left(1-P_{0}\right)\left[\frac{\Lambda Q \log _{2} e}{1-e^{-\Lambda Q}}-\log _{2}\left(1-e^{-\Lambda Q}\right)-\Lambda Q \gamma \log _{2} e+1\right]
$$

where $\Lambda=\sqrt{2} / \sigma$ is the Laplace parameter that is one-to-one mapping of $\sigma ; \gamma Q$ represents the rounding offset when $\gamma$ is a parameter between $(0,1)$, such as $1 / 6$ for H.264/AVC interframe coding [4]; $P_{0}=1-e^{-\Lambda Q(1-\gamma)}$ is the probability of quantized transform coefficient being zero. Since the source distortion is only caused by quantization error, the corresponding source distortion model is expressed as:

$$
D(\Lambda, Q)=\frac{\Lambda Q e^{\gamma \Lambda Q}(2+\Lambda Q-2 \gamma \Lambda Q)+2-2 e^{\Lambda Q}}{\Lambda^{2}\left(1-e^{\Lambda Q}\right)}
$$

The derivation process and proof of Eqs. (5) and (6) are given in Appendix A. Integrating Eq. (4) and $\Lambda=\sqrt{2} / \sigma$ into Eqs. (5) and (6), both source rate and distortion can be further expressed as functions of $\lambda$ and $Q$, i.e., $R(\lambda, Q)$ and $D(\lambda, Q)$. 


\section{B. Encoding Time and Power Model}

As introduced in [1], the encoding complexity comprises of three segments: motion estimation, PRE-coding (transform, quantization, inverse quantization and inverse transform), and entropy coding. Theoretically, the entire encoding time is the sum of individual duration of each of the three segments. In order to achieve higher compression efficiency, H.264/AVC utilizes tree structured motion compensation with seven inter- modes, which causes ME as the most time consuming part within all the three segments of the encoder. Likewise, Fig. 6 illustrates the ratio of motion estimation time to the entire encoding time for the Bus (QCIF) and Foreman (CIF) sequences, with regard to search range and number of reference frames. Here, for a specific sequence, the motion estimation time ratio is obtained by dividing the average motion estimation time by the average entire encoding time for a P-frame. It can be seen from Figs. 6(a) and 6(b) that ME takes majority of the entire encoding time, e.g. more than $90 \%$ when $\lambda$ greater than 5 and within all the range of $\theta$. Furthermore, the ratio grows and approaches to 1 with the increment of search range and number of reference frames. Thus, as demonstrated in [6], [8], it is reasonable to approximate the entire encoding time by the motion estimation time for IPPPP coding mode. It is worth mentioning that the ME time ratio throughout this paper is attained by exhaustive full search, which can guarantee achieving the optimal motion vector and minimum predictive residuals.

Technically, the motion estimation time of an inter-coded $\mathrm{P}$ frame can be derived as the total number of CPU clock cycles consumed by its SAD operation divided by the number of clock cycles per second. Namely, the encoding time for an inter-coded P frame is approximated by the motion estimation time as

$$
d(\lambda, \theta) \approx \operatorname{MET}(\lambda, \theta)=\frac{N(2 \lambda+1)^{2} \theta \cdot c_{0}}{f_{C L K}}
$$

where $N$ is the number of MBs in a frame; $(2 \lambda+1)^{2} \theta$ is the total number of SAD operations in a 3-D search cube for each $\mathrm{MB}$; $c_{0}$ is the number of clock cycles of one SAD operation over a given CPU; $f_{C L K}$ is the clock frequency of the CPU. Through the dynamic voltage scaling model to control power consumption of a microprocessor [25] [26], $f_{C L K}$ can be further related 


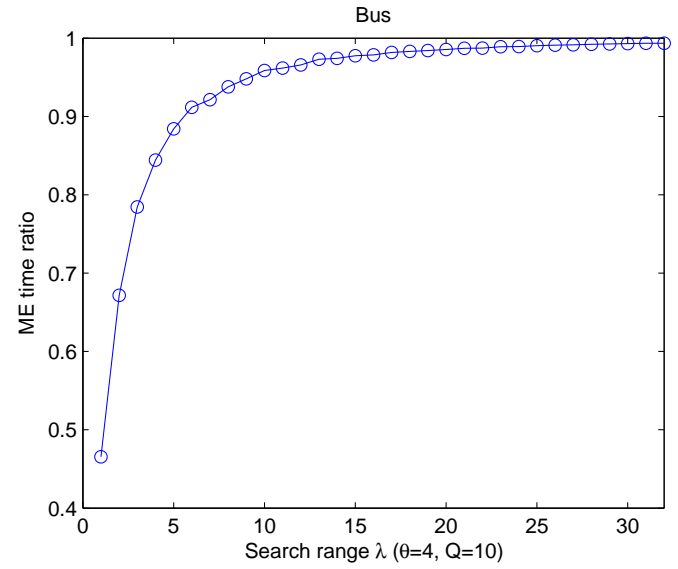

(a)

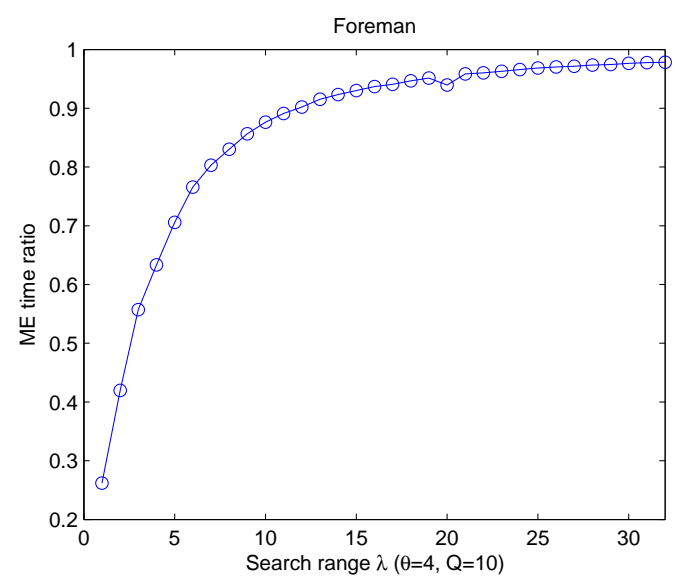

(c)

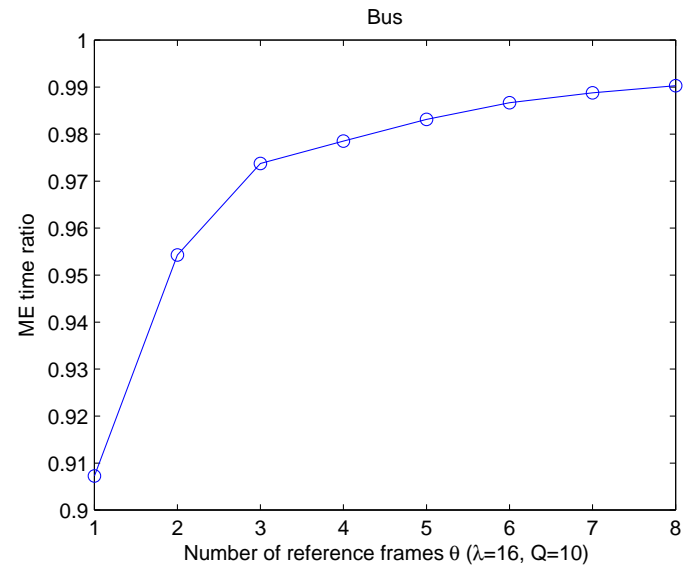

(b)

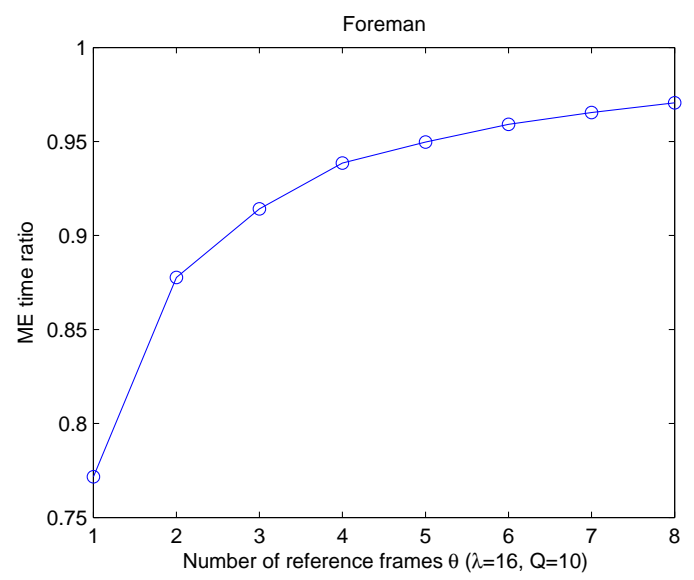

(d)

Fig. 6. Impact of search range and number of reference frames on the motion estimation time ratio, (a) search range and (b) number of reference frames of Bus video sequence, and (c) search range and (d) number of reference frames of Foreman video sequence.

to the CPU power consumption:

$$
P=k \cdot f_{C L K}^{3}
$$

where $k$ is a constant in the dynamic voltage scaling model and determined by both the supply voltage and the effective switched capacitance of the circuits [27].

To justify the theoretical encoding time model in Eq. (7), Fig. 7 is provided to further investigate the relationship between the motion estimation time (MET) and ME parameters $\lambda$, $\theta$ and quantization step size $Q$. It can be seen from Figs. 7(a) and 7(b) that MET can be fitted by a linear function of either search area $(2 \lambda+1)^{2}$ or the number of pixels ever searched in a 


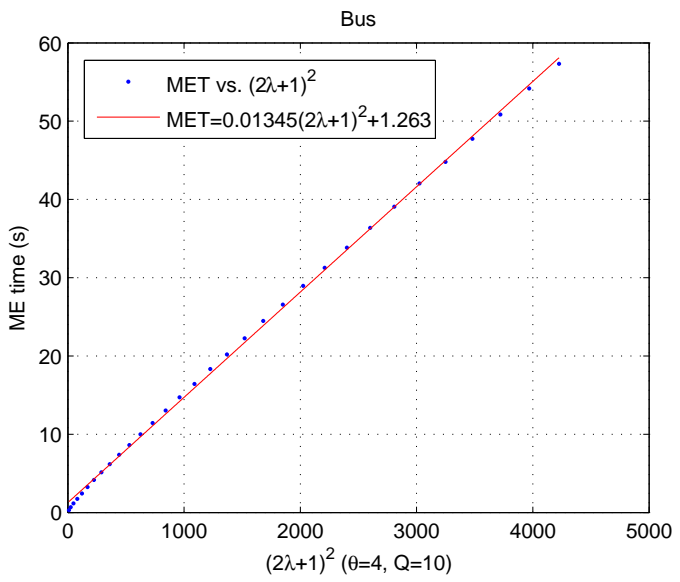

(a)

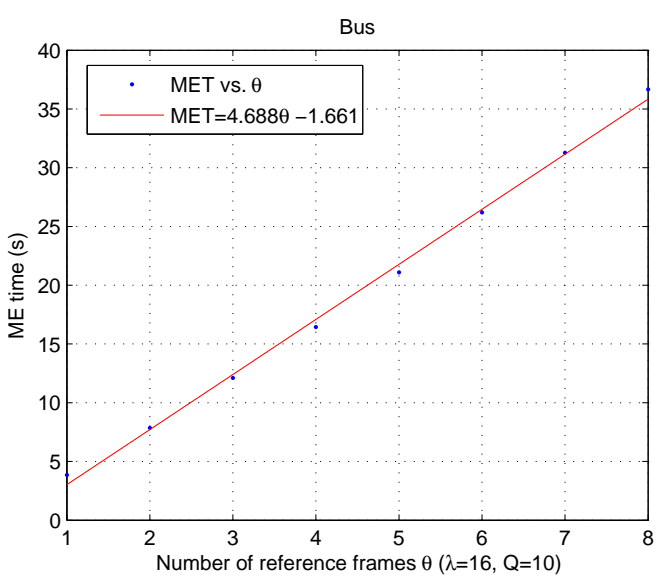

(c)

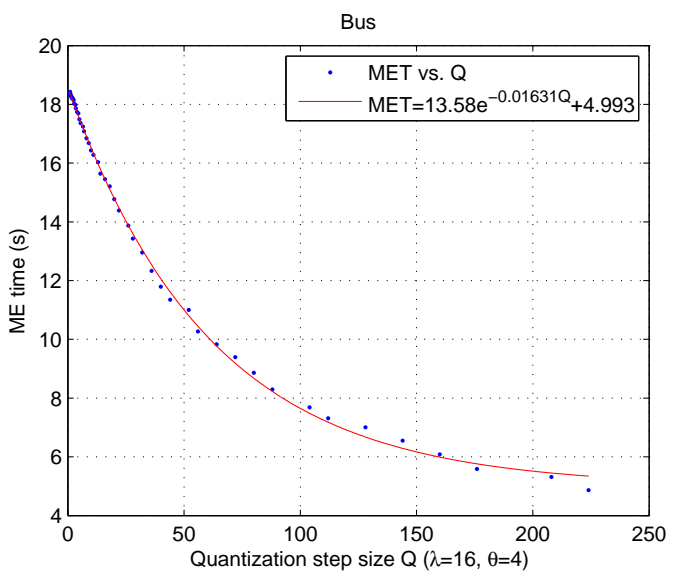

(e)

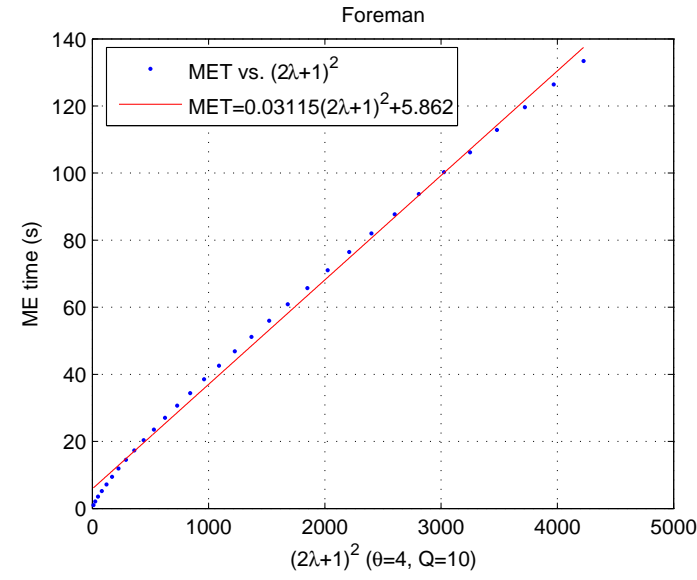

(b)

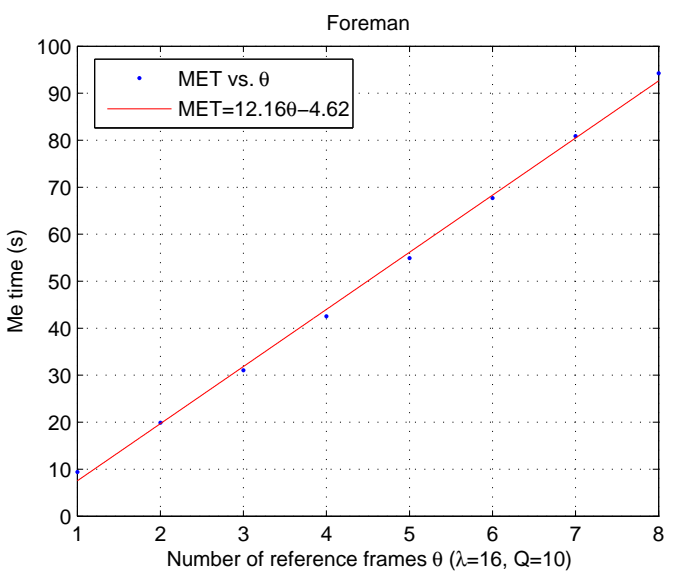

(d)

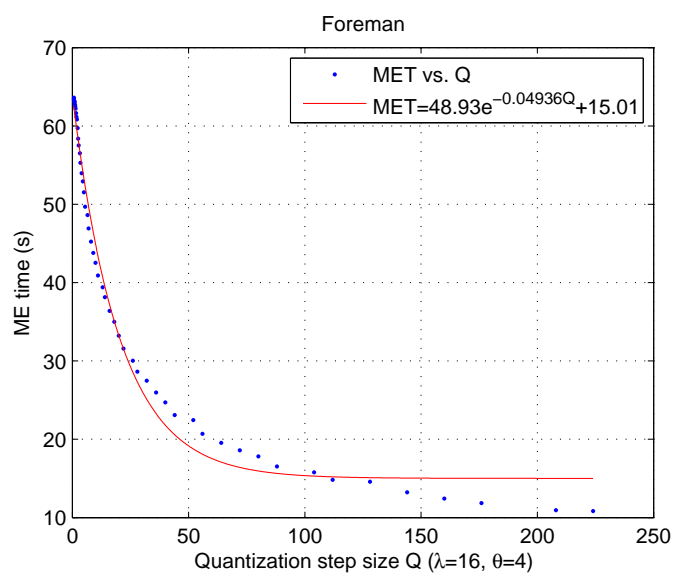

(f)

Fig. 7. Relationship and fitting results of motion estimation time vs. (a) search area, (c) number of reference frames, and (e) quantization step size for Bus video sequence, and (b) search area, (d) number of reference frames, and (f) quantization step size for Foreman video sequence. 
reference frame. Similarly, Figs. 7(c) and 7(d) indicate that MET can also be fitted by a linear function of number of reference frames $\theta$. In accordance with Eq. (7), the linear relationship between MET and $(2 \lambda+1)^{2}$ or $\theta$ is obvious, since $(2 \lambda+1)^{2} \cdot \theta$ would form a 3-D search cube for a MB in ME and represent the total number of SAD operations per MB. In Figs. 7(e) and 7(f), MET is illustrated to be dependent on quantization step size $Q$ too. The relationship can be well fitted by an exponential function with a constant translation along the MET axis. Specifically, the higher the quantization step size is, the more likely a MB would satisfy the skip mode condition and thus the more MBs would end up with skip mode as the real coding mode, which can lower the encoding complexity. The higher the quantization step size is, the fewer number of $\mathrm{SAD}$ operations for each MB is involved, and the lower complexity to encode the inter-frame. Hence, Eq. (7) is modified to reflect such dependency between the motion estimation time and the quantization step size:

$$
d(\lambda, \theta, Q) \approx \operatorname{MET}(\lambda, \theta, Q)=\frac{N(2 \lambda+1)^{2} \theta \cdot \alpha(Q) \cdot c_{0}}{f_{C L K}}
$$

where $\alpha(Q)$ denotes the ratio of the actual number of SAD operations in the JM codec to the theoretical total number of SAD operations, and thus $N(2 \lambda+1)^{2} \theta \cdot \alpha(Q)$ represents the actual number of SAD operations of a frame.

Fig. 8 illustrates the relationship of MET vs. search area and quantization step size, with number of reference frames fixed at 1 , namely, the functional form of $d(\lambda, \theta, Q \mid \theta=1)$. Comparing the two dimensional fitting results with Eq. (9), it can be observed that $\frac{N \cdot \alpha(Q) \cdot c_{0}}{f_{C L K}}=0.003612$. $\left(0.7422 e^{-0.01113 Q}+0.212\right)$, where $N=99$ for QCIF video sequence. The correctness of Eq. (9) can also be validated by the results in Fig. 7 .

\section{Model Accuracy Verification}

1) Source Rate and Distortion: According to [28], the transform coefficients in a video encoder are not i.i.d.. As described in [16], the 16 coefficients in a $4 \times 4$ integer transform show a decreasing variance in the zigzag scan order. To improve the model accuracy of source rate and distortion, the coefficients should be modeled by random variables with different variances. The joint entropy and overall quantization distortion for the 16 coefficients can be applied to the source rate and distortion models. Specifically, suppose $(x, y), x, y \in\{0,1,2,3\}$ is the position 


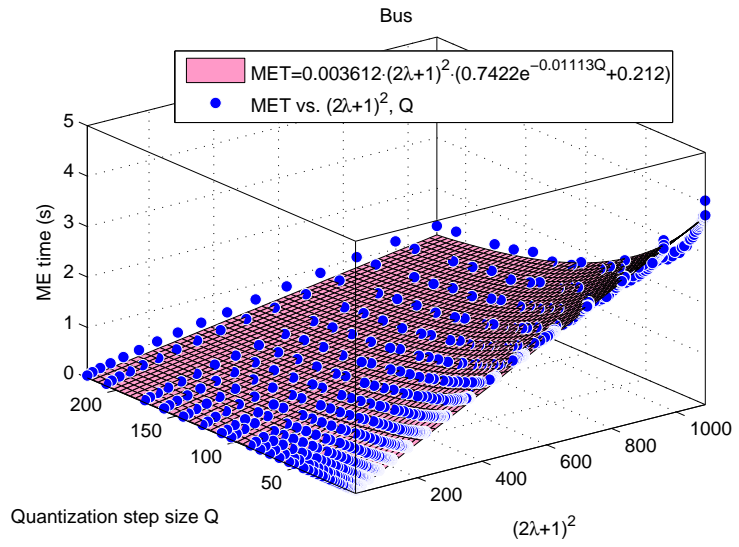

(a)

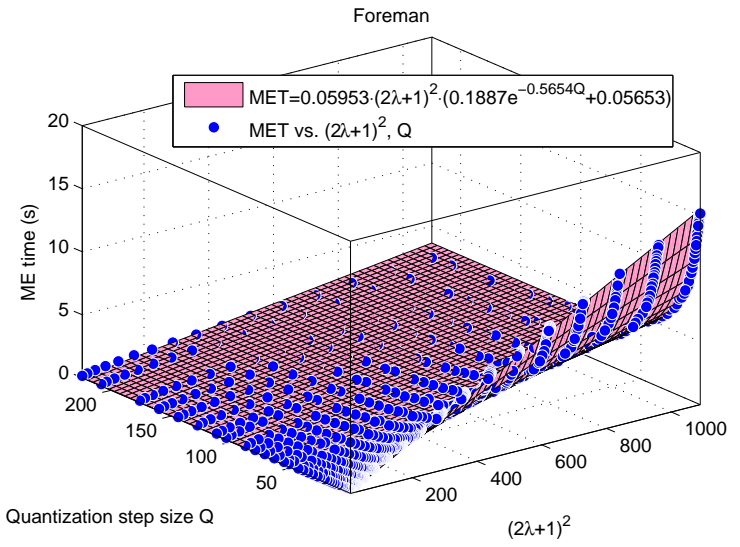

(b)

Fig. 8. Two dimensional fitting of motion estimation time vs. search area and quantization step size with $\theta=1$, (a) Bus video sequence, and (b) Foreman video sequence.

of a specific coefficient in the two-dimensional transform domain of the $4 \times 4$ integer transform, the variance $\sigma_{(x, y)}^{2}$ is derived by the average variance $\sigma^{2}$ of all positions.

$$
\sigma_{(x, y)}^{2}=2^{-(x+y)} \cdot \sigma_{(0,0)}^{2}=2^{-(x+y)} \cdot \frac{1024}{225} \sigma^{2}
$$

Therefore, the source rate and distortion model can be improved by:

$$
\begin{gathered}
R(\Lambda, Q)=H(\Lambda, Q)=\frac{1}{16} \sum_{x=0}^{3} \sum_{y=0}^{3} H(\Lambda, Q)_{(x, y)} \\
D(\Lambda, Q)=\frac{1}{16} \sum_{x=0}^{3} \sum_{y=0}^{3} D(\Lambda, Q)_{(x, y)}
\end{gathered}
$$

where $\Lambda=\sqrt{2} / \sigma$ is the Laplace parameter as defined in Eq. (5), $H(\Lambda, Q)_{(x, y)}$ and $D(\Lambda, Q)_{(x, y)}$ are the entropy and distortion associated with coefficient in position $(x, y)$, respectively.

Considering the Laplacian distribution representing the residual probability distribution may deviate significantly from the residual histogram, in addition, the mismatch would be compensated by the statistics from the previous frame [16], [6],:

$$
R_{t}^{k}=\frac{R_{t}^{k-1} R_{l}^{k}}{R_{l}^{k-1}}
$$




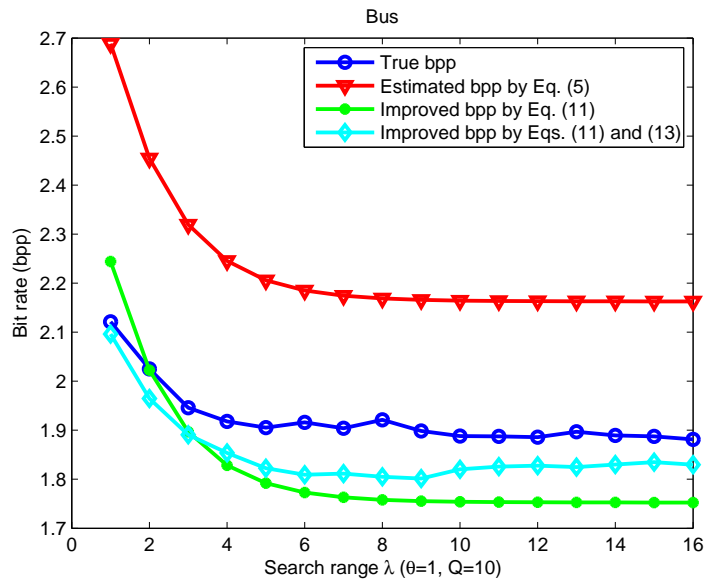

(a)

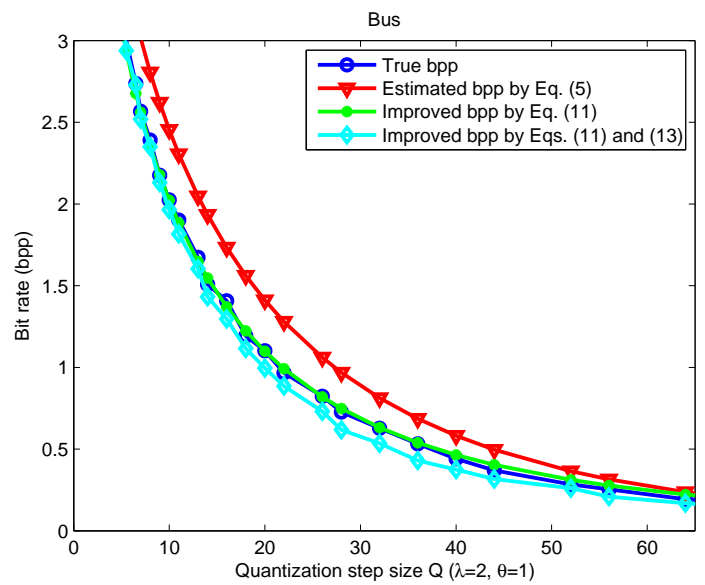

(c)

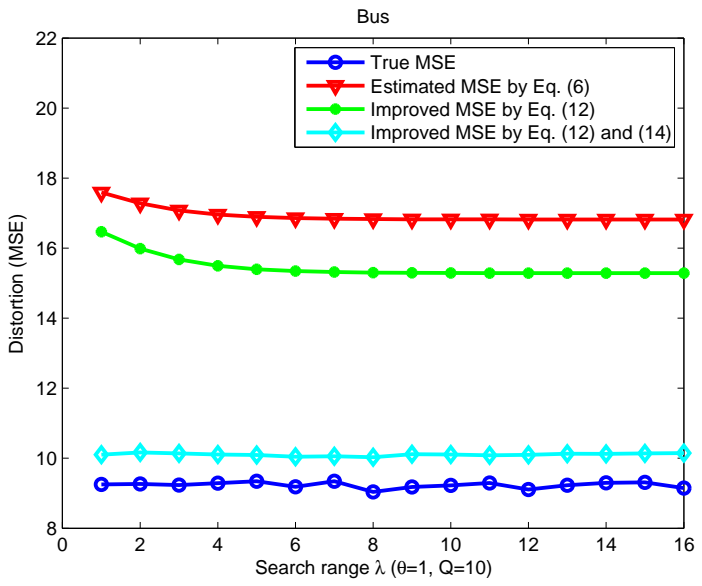

(b)

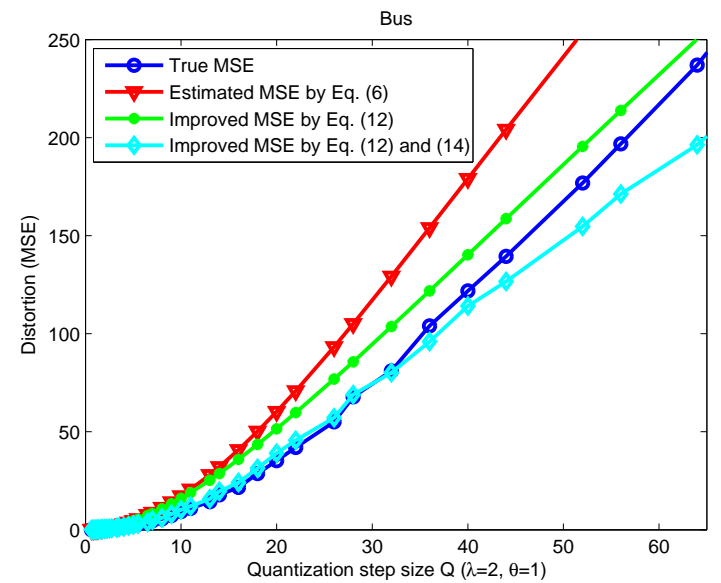

(d)

Fig. 9. Compensation results of (a) bit rate and (b) MSE vs. search range for Bus video sequence, and (c) bit rate and (d) MSE vs. quantization step size for Bus sequence.

$$
D_{t}^{k}=\frac{D_{t}^{k-1} D_{l}^{k}}{D_{l}^{k-1}}
$$

where superscripts $k-1$ and $k$ denote the frame index of two consecutive frames, subscripts $l$ and $t$ denote the estimated value under Laplacian distribution assumption and the true value, respectively.

In Figs. 9-11, the accuracy of the proposed source rate model Eq. (5) and distortion model Eq. (6) is evaluated in comparison to the actual bit per pixel (bpp) and MSE measures. In addition, the compensated model estimate of source rate and distortion on Eqs. (11) and (12), as well as the improvement on Eqs. (13) and (14), are illustrated. According to [16], in a video sequence, 


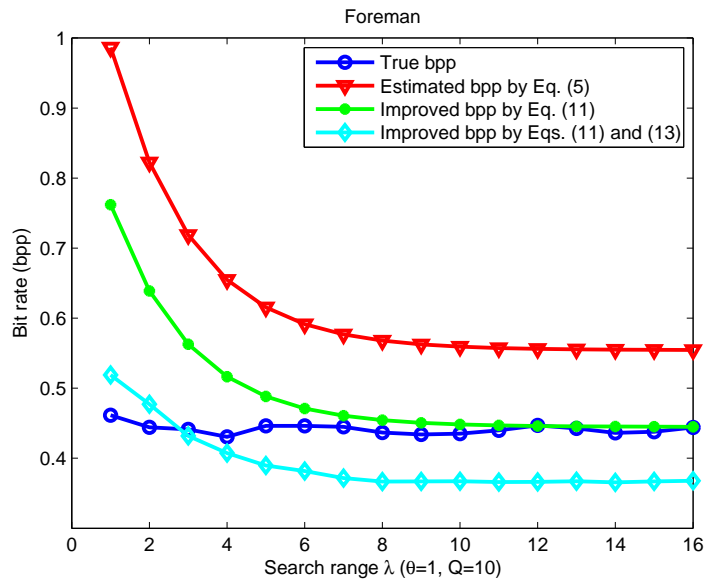

(a)

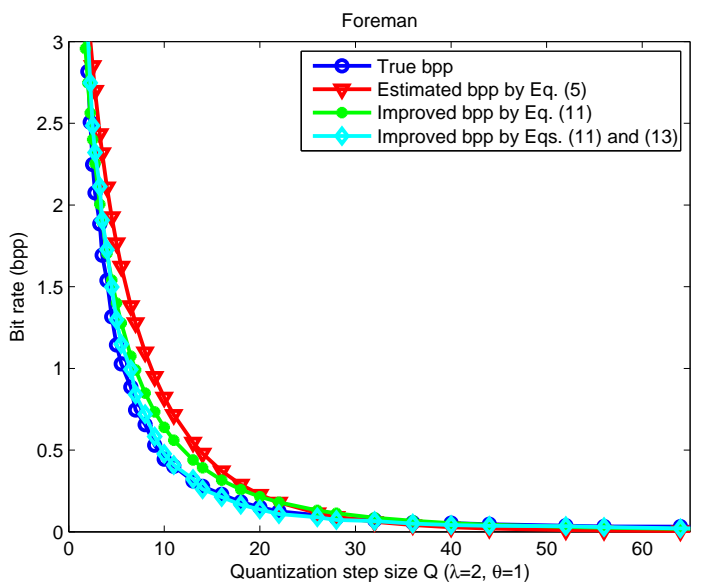

(c)

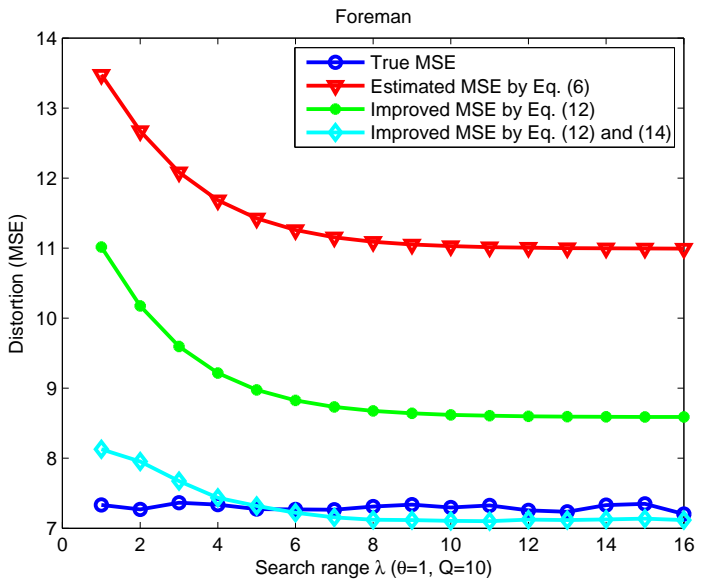

(b)

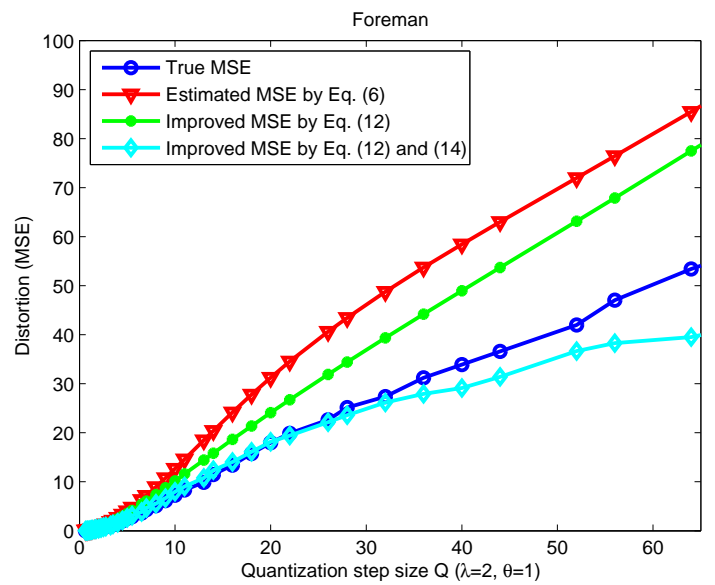

(d)

Fig. 10. Compensation results of (a) bit rate and (b) MSE vs. search range for Foreman video sequence, and (c) bit rate and (d) MSE vs. quantization step size for Foreman sequence.

the changes of residual statistics vs. $\lambda$ and $Q$ have almost the same effect on the actual values and model based values. Therefore, the accuracy of the source rate and distortion models could be improved mainly by compensating the statistics from the previous frames.

2) Encoding Time: Although the entire encoding time can be approximated by the motion estimation segment, we would modify encoding time model slightly by compensating an offset to the PRE-coding and entropy coding segments, which is much smaller compared with motion estimation time. In experiments, the off-set time of the current frame is set as the average difference time between actual encoding time and model based encoding time estimation of previous frames within a sliding window. Fig. 12 shows the true encoding time and the estimated 


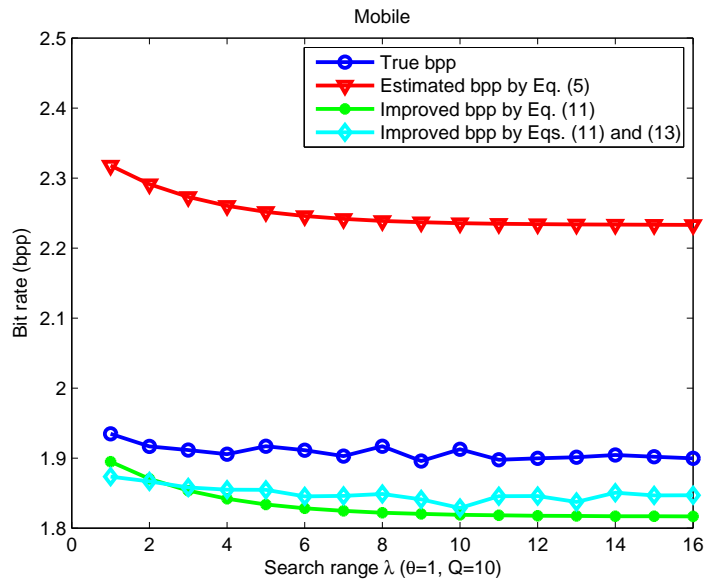

(a)

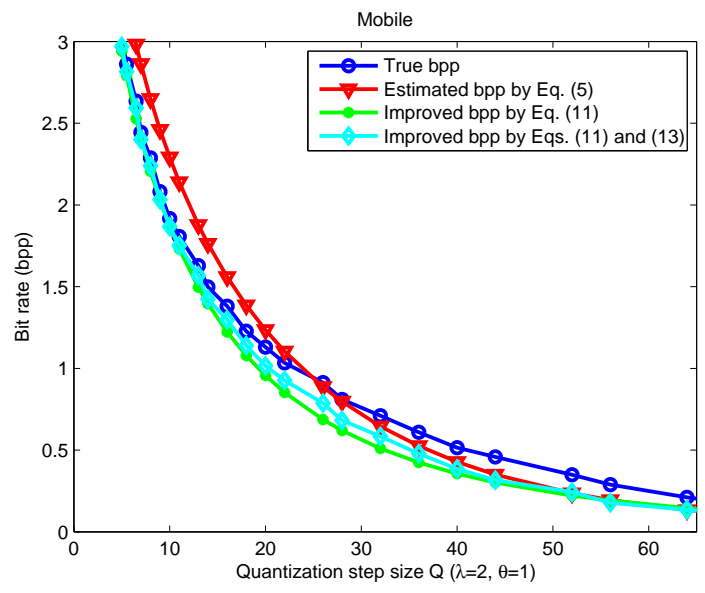

(c)

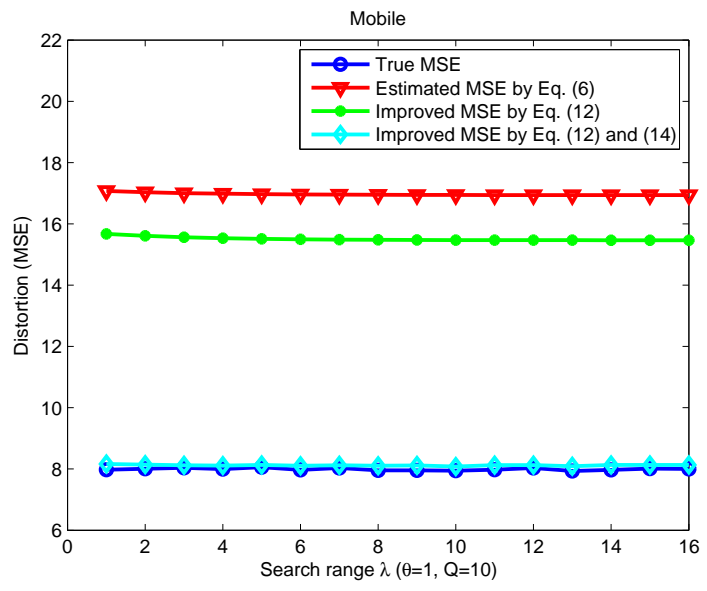

(b)

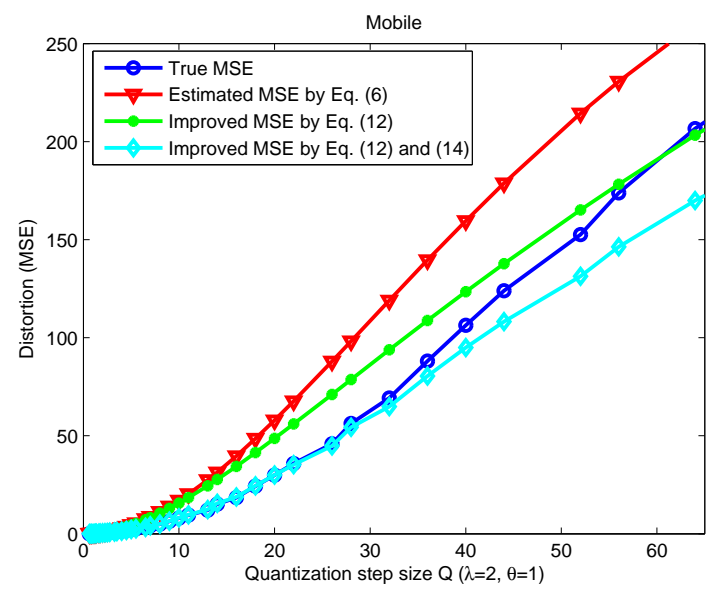

(d)

Fig. 11. Compensation results of (a) bit rate and (b) MSE vs. search range for Bus video sequence, and (c) bit rate and (d) MSE vs. quantization step size for Mobile sequence.

encoding time, which can be seen that the difference between the encoding time model Eq. (9) and its true value is relatively small. As the encoding time increases, the difference would become less significant.

\section{D-P-R-D Optimization Based Source Rate Control and Algorithm Design FOR IPPPP CODING MODE}

In this section, we apply the proposed models in Sec. II to formulate the source rate control problem as a d-P-R-D optimization with respect to search range $\lambda$ and quantization step size $Q$, and accordingly design a practical rate control algorithm. 


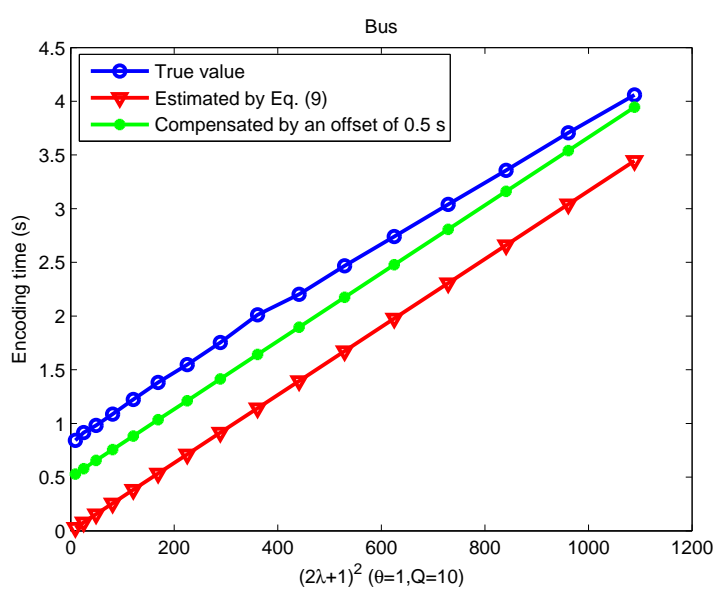

(a)

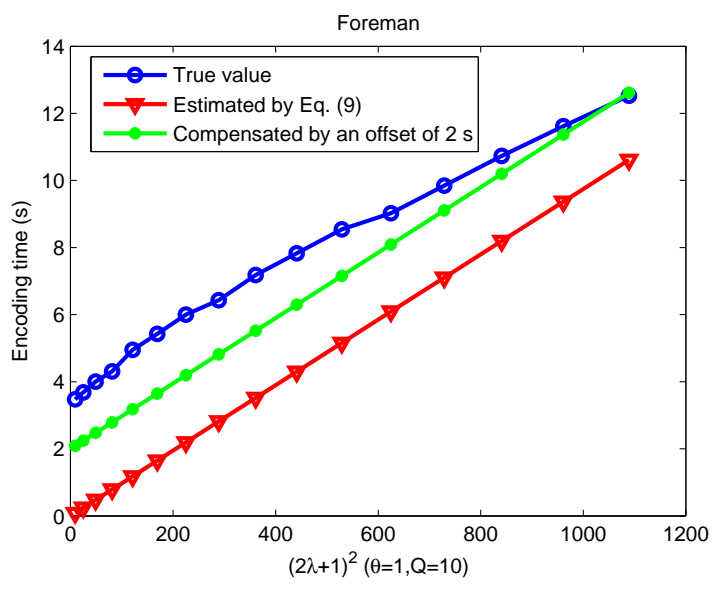

(c)

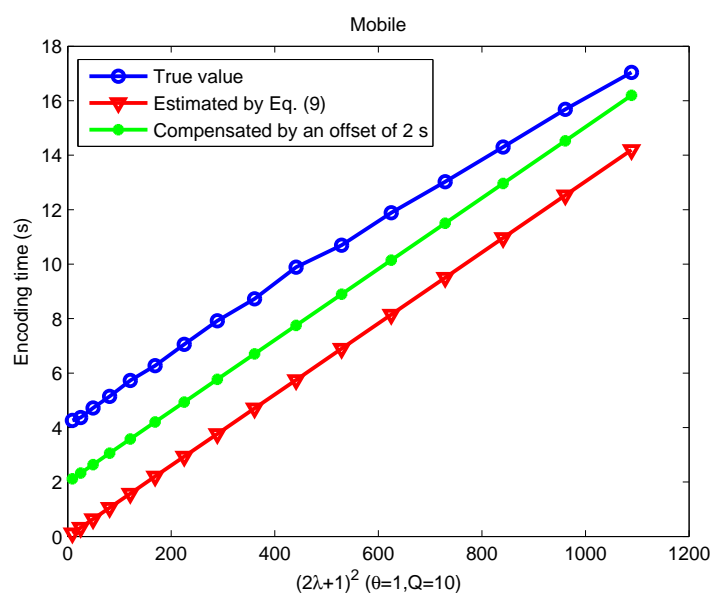

(e)

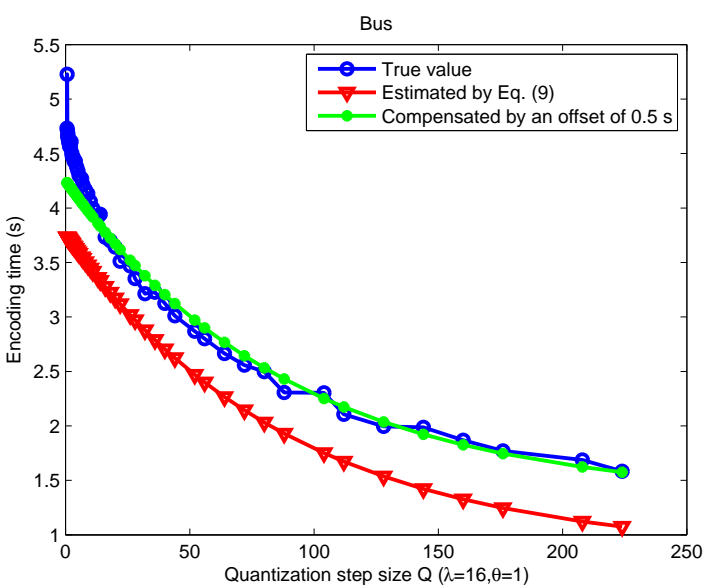

(b)

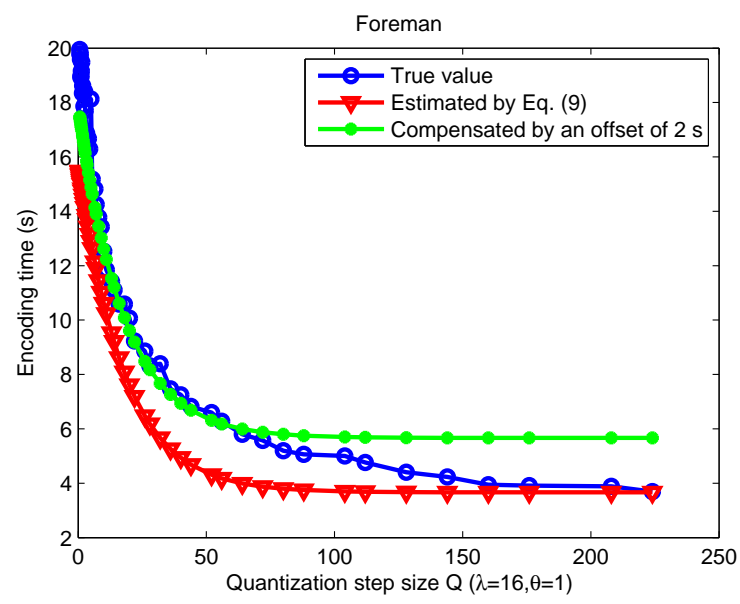

(d)

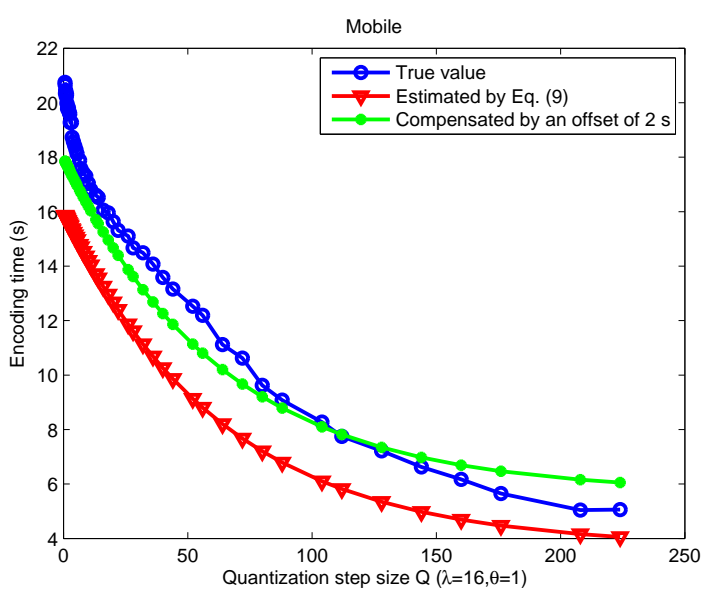

(f)

Fig. 12. Compensation results of encoding time vs. (a) search area and (b) quantization step size for Bus video sequence, and (c) search area and (d) quantization step size for Foreman video sequence, and (e) search area and (f) quantization step size for Mobile video sequence. 


\section{A. d-P-R-D Optimization Based Source Rate Control}

In the previous section, we have derived the analytical models of rate, distortion, delay and power, as functions of three parameters, search range $\lambda$, number of reference frames $\theta$, and quantization step size $Q$, respectively. As discussed in Sec. II-A, however, $\theta$ is a less effective parameter on the change of $\sigma$ than the other two parameters. To this end, we keep the value of $\theta$ fixed and choose $\lambda$ and $Q$ as the tuning parameters, and thus the d-P-R-D optimization based source rate control problem can be formulated as

$$
\begin{array}{ll}
\min & D(\lambda, Q) \\
\text { s.t. } & R(\lambda, Q) \leq R_{\max } \\
& d(\lambda, Q) \leq d_{\max } \\
& P \leq P_{\max }
\end{array}
$$

Compared with the existing works on source rate control problems, the optimization problem Eq. (15) is constrained by two more conditions of the encoding time and encoding power, in addition to the rate. Ideally, an efficient video encoder is expected to encode a raw video sequence into a bit stream with minimum distortion, rate, encoding delay and encoding power. From the analysis of the proposed d-P-R-D models in Sec. II, it can be seen that a larger search range $\lambda$ as well as a smaller quantization step size $Q$ is required to achieve the objective of minimizing the distortion $D(\lambda, Q)$. On the other hand, however, the decrement of $Q$ will cause the rate $R(\lambda, Q)$ to increase, and the encoding delay $d(\lambda, Q)$ will also become greater with either $\lambda$ increasing or $Q$ decreasing. Furthermore, for a coding parameter pair $(\lambda, Q)$, the encoding delay $d(\lambda, Q)$ can

be further reduced by increasing the encoding power $P$, while the distortion and rate are still kept at the same level. Therefore, it is infeasible for a video encoder to simultaneously achieve the goals of minimizing distortion, rate, encoding delay and encoding power. Accordingly, the d-P-R-D optimization based source rate control problem Eq. (15) is to find the Pareto optimal tradeoff among the four optimization criteria. As a matter of fact, the target of such optimization is to minimize the distortion $D(\lambda, Q)$ for given rate $R_{\max }$, given encoding delay $d_{\max }$ and given encoding power $P_{\max }$, by appropriate selections of coding parameter pair $(\lambda, Q)$.

Depending on the estimation accuracy of the d-P-R-D models, the source rate control problem 
Eq. (15) can be applied to a desired coding unit, e.g. a sequence, a group of pictures (GOP), a frame, or a macroblock. For example, if the d-P-R-D model is applied to a stream, Eq. (15) can be regarded to solve the sequence level rate control problem. If it is applied to a frame, Eq. (15) can behave as a frame level rate control. Without loss of generality, a sequence level rate control problem will be imposed on Eq. (15) with a practical solution.

\section{B. Algorithm Design}

Considering both Eqs. (8) and (9), for the coding parameter pair $(\lambda, Q)$, the minimum encoding delay would be achieved with the maximal power $P_{\max }$. In other words, the feasible set of coding parameters $(\lambda, Q)$ constrained by a given maximum encoding delay would become the largest if and only if the encoding power reaches the maximum. According to Proposition 1, the power constraint (15d) in (15) is therefore an active constraint at the optimal coding parameter pair $\left(\lambda^{*}, Q^{*}\right)$, and the source rate control problem Eq. (15) can be transformed to an equivalent problem Eq. (16).

Proposition 1. Problem (15) is equivalent to the following optimization problem:

$$
\begin{array}{ll}
\min & D(\lambda, Q) \\
\text { s.t. } & R(\lambda, Q) \leq R_{\max } \\
& d(\lambda, Q) \leq d_{\max } \\
& P=P_{\max }
\end{array}
$$

Proof: Please refer to [29].

Either the Lagrange multiplier method [30] [31] [32] or the dynamic programming approach [33] can solve Eq. (16). The former is preferred throughout this paper since it can be implemented independently in each coding unit. In comparison, the dynamic programming approach requires a tree representing all possible solutions to grow over multiple coding units. The computational complexity would grow exponentially with the number of coding units, which is not affordable for practical applications. With the Lagrange multiplier method, Eq. (16) can be converted to an unconstrained problem Eq. (17):

$$
\min \quad L(\lambda, Q, \mu, \eta)=D(\lambda, Q)+\mu \cdot\left[R(\lambda, Q)-R_{\max }\right]+\eta \cdot\left[d(\lambda, Q)-d_{\max }\right]
$$


where $\mu \geq 0$ and $\eta \geq 0$ are Lagrange multipliers associated with two inequality constraints, and the equality constraint $P=P_{\max }$ can be integrated to $d(\lambda, Q)$ as:

$$
d(\lambda, Q)=\frac{N(2 \lambda+1)^{2} \theta \cdot \alpha(Q) \cdot c_{0}}{\sqrt[3]{k^{-1} P_{\max }}}
$$

Based on the theorem in [30], we have the following theorem that relates the solution to the unconstrained problem Eq. (17) to the solution to the constrained problem Eq. (16).

Theorem 1. For any $\mu \geq 0, \eta \geq 0$, the solution $\left(\lambda^{*}, Q^{*}\right)$ to the unconstrained problem Eq. (17) is also the solution to the constrained problem Eq. (16) with the constraints $R(\lambda, Q) \leq R\left(\lambda^{*}, Q^{*}\right)$ and $d(\lambda, Q) \leq d\left(\lambda^{*}, Q^{*}\right)$.

Proof: Please refer to [32].

It should be noted that although Theorem 1 does not guarantee any solution to the constrained problem Eq. (16), it indicates that for any nonnegative $\mu$ and $\eta$, there is a corresponding constrained problem whose solution is identical to that of the unconstrained problem. Therefore, as $\mu$ and $\eta$ are swept over the range $[0,+\infty]$, if there exists a specific pair of $\mu^{*}$ and $\eta^{*}$ which makes $R\left(\lambda^{*}, Q^{*}\right)$ and $d\left(\lambda^{*}, Q^{*}\right)$ happen to be equal to $R_{\max }$ and $d_{\max }$, then $\left(\lambda^{*}, Q^{*}\right)$ is the desired solution to the constrained problem Eq. (16).

To estimate the corresponding $\mu^{*}$ and $\eta^{*}$ in practice, the bi-section search method [16] [32] is commonly used for iterations to acquire the best Lagrange multiplier. However, two disadvantages have prevented such method from being suitably applied to the unconstrained problem Eq. (17). First, the bi-section search method would perform worse or even fail to get the solution when extended to two dimensional search scenario. For example, if we simultaneously bisect the intervals for $\mu$ and $\eta$ and then select a subinterval for each of these two Lagrange multipliers based on their own criteria, respectively, the best solution $\mu^{*}$ and $\eta^{*}$ might be excluded by such independent bisections. Therefore, in order to get the best solution, in many cases we have to implement an exhausting search over two dimensions, which is very time consuming. Second, even if the bi-section search can suitably work for searching two Lagrange multipliers simultaneously, we still need to update the Lagrange multipliers in each iteration, and then solve the corresponding unconstrained problem Eq. (17) to get the solution. It means the update of primal and dual variables are not synchronous and may cause high computational complexity. 
Another analytical way to determine the best Lagrange multiplier is the model-based method [4] [31], which focuses on rate-distortion optimization and accordingly derives an accurate function between the single Lagrange multiplier and $Q$. Without consideration of the encoding time and power, however, the derived function is no longer accurate and thus cannot be directly applied to the d-P-R-D optimization.

In the following, we propose a practical algorithm for the d-P-R-D optimization based source rate control problem Eq. (16) on the basis of KKT conditions and sequential quadratic programming (SQP) methods, which can produce both primal $\left(\lambda^{*}\right.$ and $\left.Q^{*}\right)$ and dual (Lagrange multipliers $\mu^{*}$ and $\eta^{*}$ ) solutions simultaneously in an iterative way. To solve the first-order necessary conditions of optimality for problem Eq. (16), the SQP methods [34] [35] can be used to construct a quadratic programming subproblem at a given approximate solution, and then to employ the solution to this subproblem to construct a better approximation. This process is iterated to create a sequence of approximations that is expected to converge to the optimal solution $\left(\lambda^{*}, Q^{*}, \mu^{*}, \eta^{*}\right)$. Specifically, given an iterate $\left(\lambda^{k}, Q^{k}, \mu^{k}, \eta^{k}\right)$, a new iterate $\left(\lambda^{k+1}, Q^{k+1}, \mu^{k+1}, \eta^{k+1}\right)$ can be obtained by solving a quadratic programming (QP) minimization subproblem given by:

$$
\begin{aligned}
\min _{\delta^{k}} & \frac{\partial D\left(\lambda^{k}, Q^{k}\right)}{\partial \lambda} \cdot \delta_{\lambda}^{k}+\frac{\partial D\left(\lambda^{k}, Q^{k}\right)}{\partial Q} \cdot \delta_{Q}^{k}+\frac{1}{2} \delta^{k^{T}} \cdot \nabla^{2} L\left(\lambda^{k}, Q^{k}, \mu^{k}, \eta^{k}\right) \cdot \delta^{k} \\
\text { s.t. } & \frac{\partial R\left(\lambda^{k}, Q^{k}\right)}{\partial \lambda} \cdot \delta_{\lambda}^{k}+\frac{\partial R\left(\lambda^{k}, Q^{k}\right)}{\partial Q} \cdot \delta_{Q}^{k}+R\left(\lambda^{k}, Q^{k}\right)=R_{\max } \\
& \frac{\partial d\left(\lambda^{k}, Q^{k}\right)}{\partial \lambda} \cdot \delta_{\lambda}^{k}+\frac{\partial d\left(\lambda^{k}, Q^{k}\right)}{\partial Q} \cdot \delta_{Q}^{k}+d\left(\lambda^{k}, Q^{k}\right)=d_{\max }
\end{aligned}
$$

where the derivative operators $\nabla^{2}$ is used to refer to the second-order Hessian matrix with respect to primal variables $\lambda$ and $Q$, and $\delta^{k}=\left(\delta_{\lambda}^{k}, \delta_{Q}^{k}\right)^{T}=\left(\lambda^{k+1}-\lambda^{k}, Q^{k+1}-Q^{k}\right)^{T}$ is the vector representing the update directions of primal variables. For details about the derivation of Eq. (19), please refer to Appendix B.

The aforementioned SQP algorithm, though can be used to appropriately solve Eq. (16), suffers from two deficiencies similar to the Newton's method. First, it requires at each iteration the calculation of second-order Hessian matrix $\nabla^{2} L\left(\lambda^{k}, Q^{k}, \mu^{k}, \eta^{k}\right)$, which could be a costly computational burden and in addition might not be positive definite. To address this issue, we can use the quasi-Newton method instead to construct an approximate Hessian matrix $B^{k}$ by which $\nabla^{2} L\left(\lambda^{k}, Q^{k}, \mu^{k}, \eta^{k}\right)$ is replaced in Eq. (19). In practice, such an approximation $B^{k}$ can be 
obtained by the Broyden-Fletcher-Goldfarb-Shanno (BFGS) method [36]:

$$
B^{k+1}=B^{k}+\frac{\gamma^{k} \gamma^{k^{T}}}{\gamma^{k^{T}} \delta^{k}}-\frac{B^{k} \delta^{k} \delta^{k^{T}} B^{k^{T}}}{\delta^{k^{T}} B^{k} \delta^{k}}
$$

with $\gamma^{k}$ defined by

$$
\gamma^{k}=\nabla L\left(\lambda^{k+1} Q^{k+1}, \mu^{k+1}, \eta^{k+1}\right)-\nabla L\left(\lambda^{k}, Q^{k}, \mu^{k+1}, \eta^{k+1}\right)
$$

Second, in order to have global convergence performance, a line search method [35] is used to replace the full Newton step $\left(\lambda^{k+1}, Q^{k+1}\right)^{T}=\left(\lambda^{k}, Q^{k}\right)^{T}+\delta^{k}$ by $\left(\lambda^{k+1}, Q^{k+1}\right)^{T}=\left(\lambda^{k}, Q^{k}\right)^{T}+\alpha \cdot \delta^{k}$, which defines the $\mathfrak{l}_{1}$ merit function as

$$
\mathfrak{l}_{1}(\lambda, Q)=D(\lambda, Q)+\theta \cdot\left[\max \left\{0, R(\lambda, Q)-R_{\max }\right\}+\max \left\{0, d(\lambda, Q)-d_{\max }\right\}\right]
$$

where $\theta$ is a positive penalty parameter and the step size $\alpha$ is chosen such that the $\mathfrak{l}_{1}$ merit function is reduced,

$$
\mathfrak{l}_{1}\left(\left(\lambda^{k}, Q^{k}\right)^{T}+\alpha \cdot \delta^{k}\right)<\mathfrak{l}_{1}\left(\left(\lambda^{k}, Q^{k}\right)^{T}+\delta^{k}\right)
$$

Considering both of the above modifications, an improved SQP algorithm is proposed for the d-P-R-D optimization based source rate control problem, as illustrated by Algorithm 1. Since the optimal search range and quantization step size solved by the improved SQP algorithm are continuous values and at most time may not correspond to the integer values of search range and quantization parameter, a rounding-off decision step is introduced following the SQP algorithm to find the optimal pair of discrete (feasible) search range and quantization parameter. The computational complexity comparison between the proposed algorithm and the bi-section search algorithm in [16] will be described in Sec. IV-A.

Since most wireless video communication systems require low end-to-end delay, the video encoding mode is often adopted as IPPPP coding mode. And this paper mainly focuses on deriving the analytic d-P-R-D model and model based rate control scheme for such coding mode. The proposed d-P-R-D model can be extended to a different coding mode, such as IBPBP coding mode, where the proposed model is still applicable to P-frames. However, the d-P-R-D model of B-frames needs to be reformulated in a similar way by considering the different temporal prediction distances for different B-frames. 


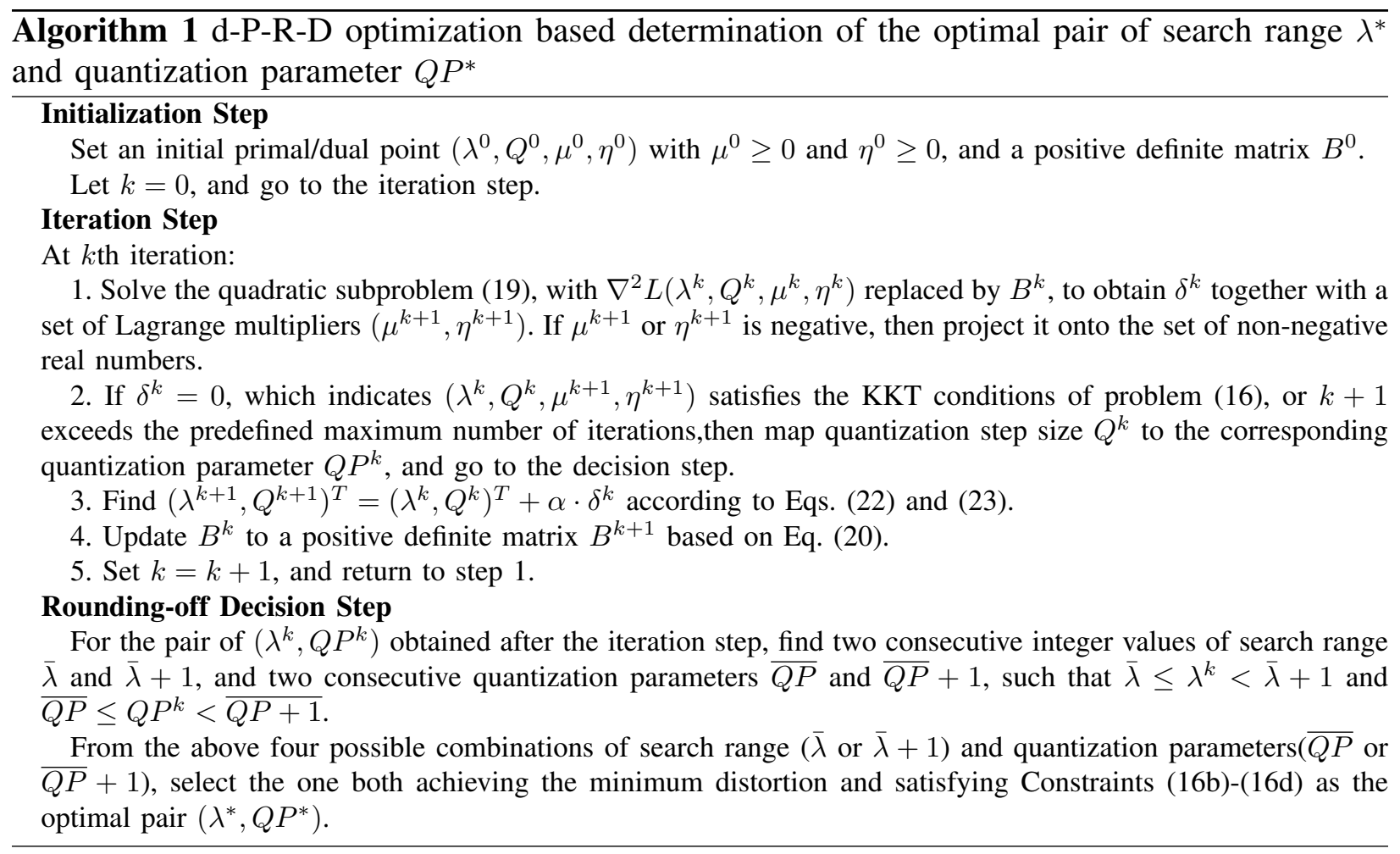

\section{EXPERIMENTAL RESULTS}

To evaluate the performance of the proposed d-P-R-D model and the d-P-R-D optimization based source rate control algorithm, we implement the proposed d-P-R-D model and associated source rate control algorithm in JM18.2 [24] encoder, with test video sequences Bus (QCIF), Foreman (CIF), Mobile (CIF), and Coastguard (CIF) at 30 frames/s, the IPPPP GOP structure, CABAC entropy coding, the maximum search range 16 of motion estimation, the dynamic range 0 to 51 of quantization parameter, and one reference frame. Since $\theta$ has much less effect on the change of $\sigma$ than $\lambda$ and $Q$, by fixing its value, the computational complexity for fitting the standard deviation function $\sigma(\lambda, \theta, Q)$ would be decreased. Furthermore, the reason to adopt only one reference frame is that the encoding time is a linear function of $\theta$, and with $\theta=1$ the encoding time is the minimum among different selections of $\theta$ while the impact of $\theta$ on the source rate and distortion is little. The maximum power consumption level is measured in the percentage of the maximum power consumption $P_{0}$ of the video encoder. 


\section{A. Convergence Behavior and Model Accuracy}

Figs. 13 and 14 show the convergence behavior of the proposed d-P-R-D optimization based source rate control (RC) algorithm for the first 10 frames of a video sequence. Here, the maximum power consumption level is set to $100 \%$, i.e., $P_{\max }=P_{0}$. It can be seen that by the proposed RC algorithm, both primal $(\lambda, Q)$ and dual variables $(\mu, \eta)$ can simultaneously and quickly converge to the corresponding optimal values in a few iterations. If the initial values of $\lambda$ and $Q$ are closer to the optimal solution, fewer number of iterations is needed for convergence. Within each iteration, on the other hand, it is only required to solve a quadratic programming optimization problem. In comparison, with the bi-section search algorithm [16], both the feasible region of dual variables $\mu$ and $\eta$ need an iterative bi-section search, which greatly increases the number of iterations. Within each iteration of the bi-section search algorithm, the entire feasible sets of primal variables $\lambda$ and $Q$ are exhaustively searched in order to find the optimal solution, which means that the duration of one iteration is much longer than that of the proposed RC algorithm. Take Fig. 13 as an example, with the proposed RC algorithm, only 5 iterations are needed to converge to the optimal solution under three different initialization configurations. It is observed from experiments on Bus sequence that, when the feasible ranges for $\mu$ and $\eta$ are both set to $[0,50], 13$ iterations are required for convergence with bi-section search algorithm. Furthermore, the number of iterations for convergence would become larger when the upper-bounds for $\mu$ and $\eta$ increase. Therefore, the computational complexity of the proposed RC algorithm is much lower than the bi-section search algorithm.

In Fig. 13, the maximum source bit rate and encoding delay for Bus video sequence are set to $1 \mathrm{bpp}$ and $2.5 \mathrm{~s}$, respectively. After the rounding-off decision of the proposed RC algorithm, the minimum achievable distortion is 30.83 with the optimal parameters $\lambda^{*}=12$ and $Q P^{*}=29$ $\left(Q^{*}=18\right)$. As validation, when the feasible sets of search range and QP are exhaustively searched for the first 10 frames, the minimum distortion 31.35 is achieved with optimal parameters $\lambda^{*}=11$ and $Q P^{*}=29\left(Q^{*}=18\right)$. Similarly, the maximum source bit rate and encoding delay for the Foreman video sequence in Fig. 14 are set to $0.1 \mathrm{bpp}$ and $2.5 \mathrm{~s}$, respectively. The minimum distortion by the proposed $\mathrm{RC}$ algorithm is 23.47 with $\lambda^{*}=2$ and $Q P^{*}=32\left(Q^{*}=26\right)$, while the true distortion obtained by the exhaustive search for the first 10 frames is 21.14 with $\lambda^{*}=1$ and $Q P^{*}=32\left(Q^{*}=26\right)$. Therefore, the proposed $\mathrm{RC}$ algorithm can achieve the near-optimal 


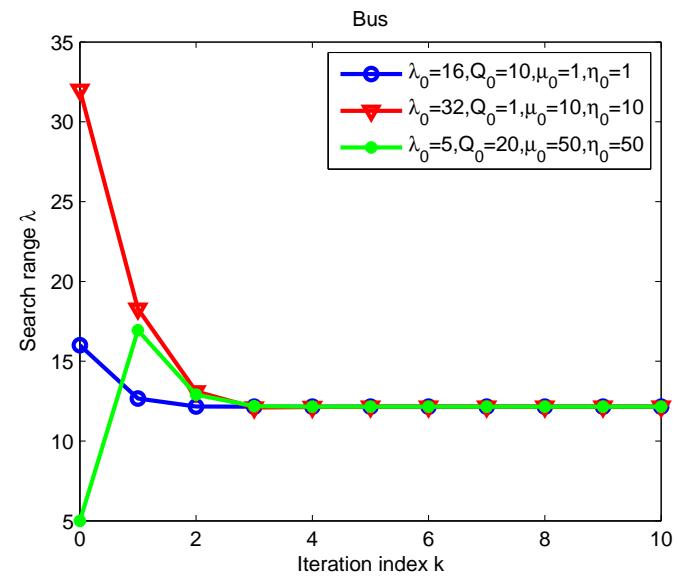

(a)

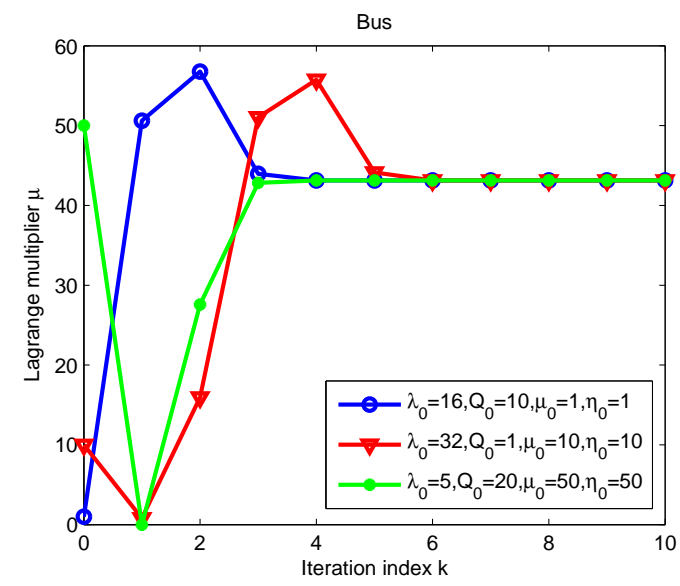

(c)

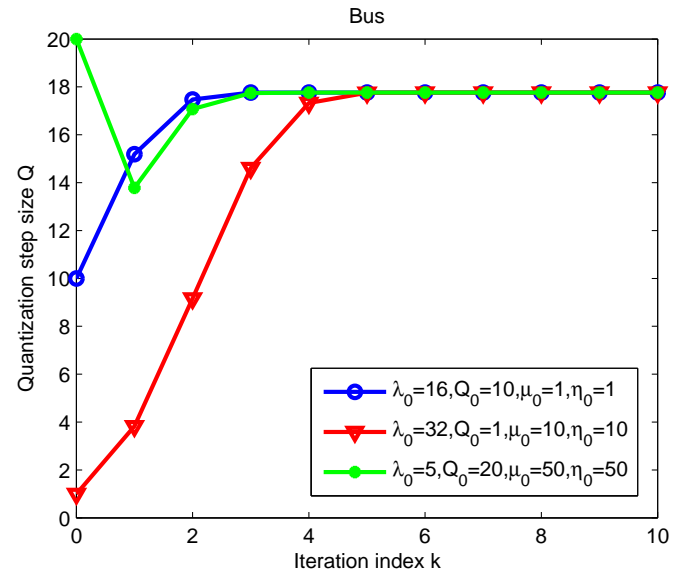

(b)

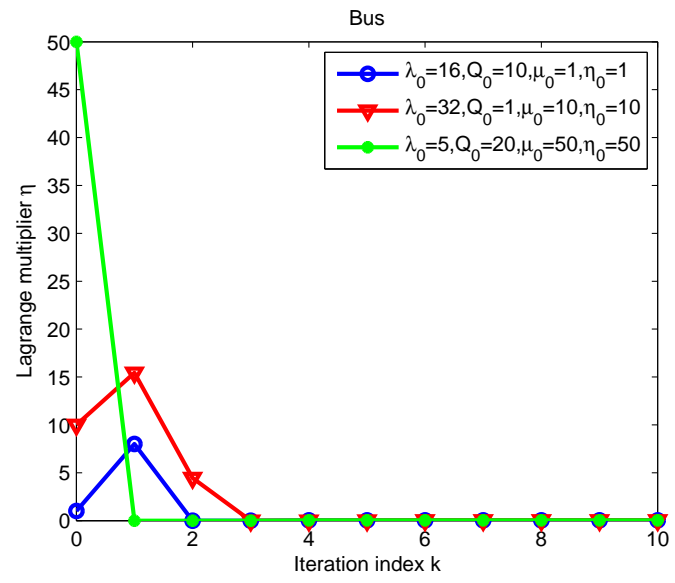

(d)

Fig. 13. Convergence behavior of (a) search range $\lambda$, (b) quantization step size $Q$, and Lagrange multipliers (c) $\mu$ and (d) $\eta$ for Bus sequence, where $\theta=1, R_{\max }=1 \mathrm{bpp}, d_{\max }=2.5 \mathrm{~s}$, and $P_{\max }=P_{0}$ is the maximum power consumption level of the video encoder, with three different sets of initial values.

performance in practice. Comparing Figs. 13 and 14, it can be further observed that for the same encoding delay constraint, a greater source bit rate is taken to encode Bus sequence yet yields a higher distortion than Foreman sequence, which is derived from the fact that the motion in Bus sequence is faster than Foreman sequence.

Fig 15 illustrates the true 3-D Pareto surface and the estimated 3-D Pareto surface by the proposed RC algorithm of source distortion $D$, source rate $R$ and encoding time $d$, when the maximum power consumption level is set to $100 \%$. A point on the 3-D Pareto surfaces indicates the minimum achievable distortion associated with given rate and encoding time constraints. It 


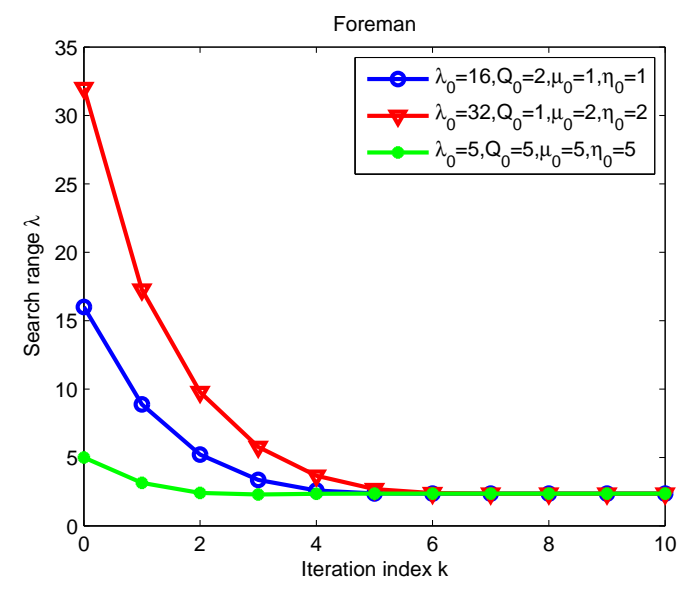

(a)

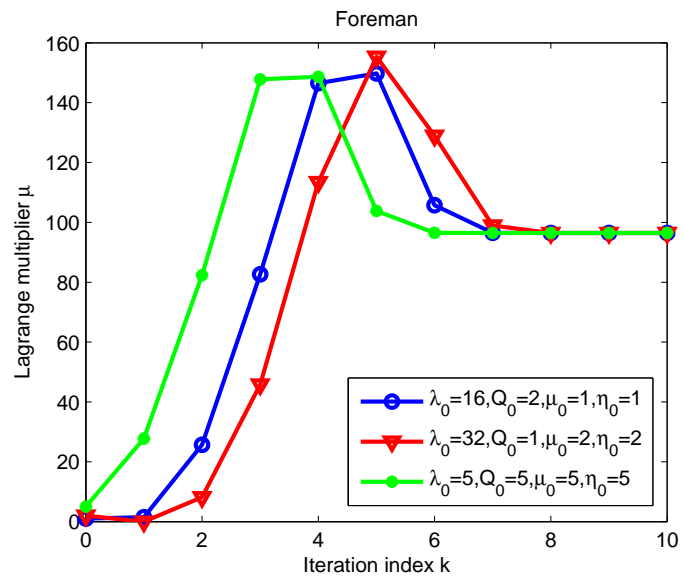

(c)

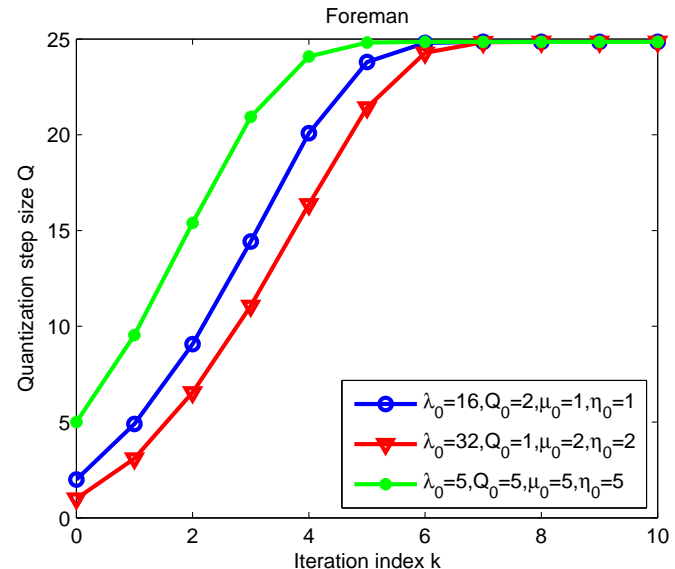

(b)

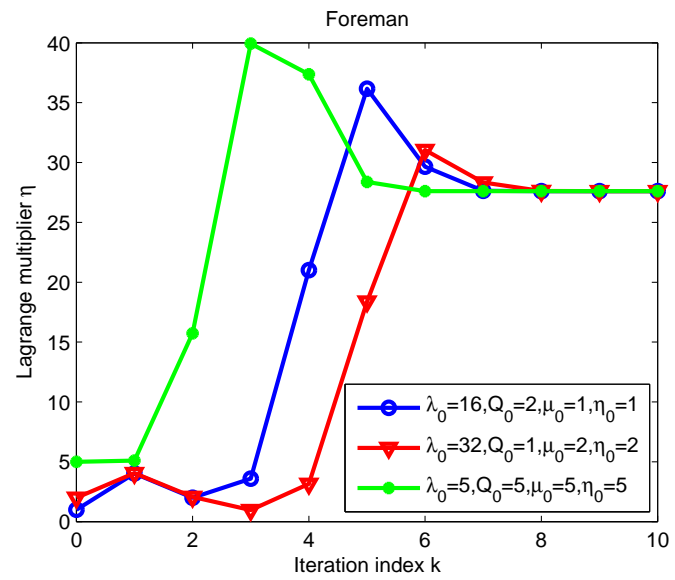

(d)

Fig. 14. Convergence behavior of (a) search range $\lambda$, (b) quantization step size $Q$, and Lagrange multipliers (c) $\mu$ and (d) $\eta$ for Foreman sequence, where $\theta=1, R_{\max }=0.1 \mathrm{bpp}, d_{\max }=2.5 \mathrm{~s}, P_{\max }=P_{0}$ is the maximum power consumption level of the video encoder, with three different sets of initial values.

can also be seen that the model estimation of the proposed $\mathrm{RC}$ algorithm is quite accurate.

\section{B. d-P-R-D Model Analysis}

To view the proposed d-P-R-D model in more detail, Fig. 16 illustrates the D-R curves for different maximum encoding times, and D-d curves for different maximum source bit rates, when the maximum power consumption level is set to $100 \%$. As the D-R curves in Figs. 16(a) and 16(c), for a given $d_{\max }, D_{\min }$ is a decreasing function of $R_{\max }$ and such curve becomes flat when $R_{\max }$ is relatively large, which corresponds to Shannon's source coding theory [37]. It is noted that the previous work on rate control shows only one D-R curve of the similar shape 


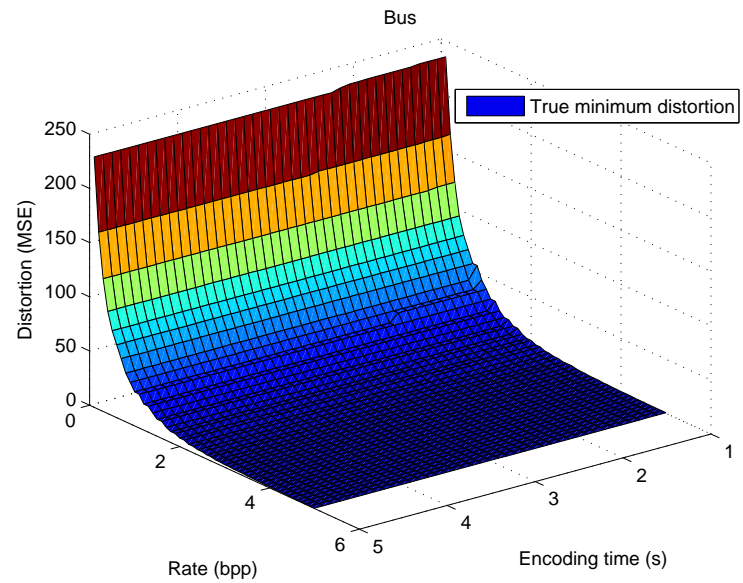

(a)

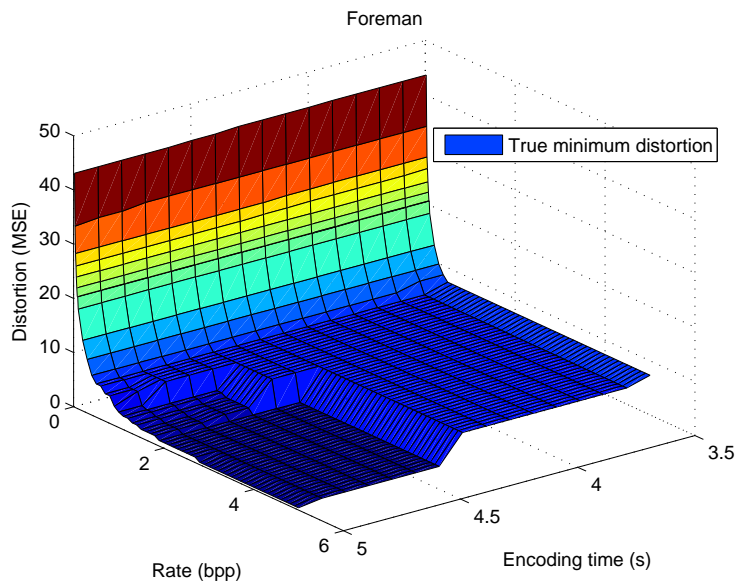

(c)

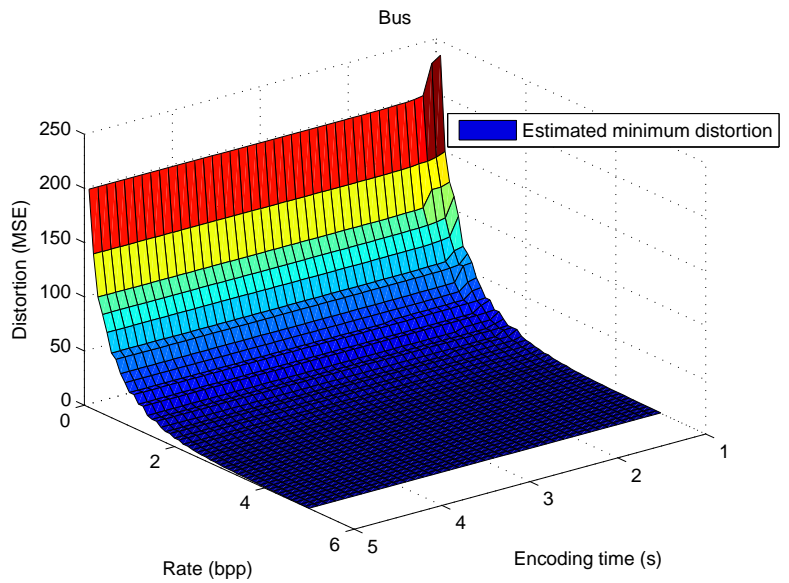

(b)

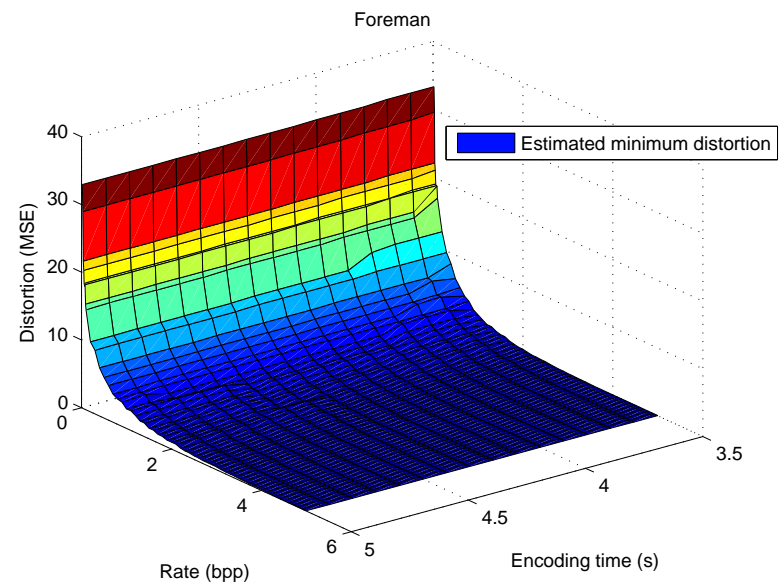

(d)

Fig. 15. 3-D Pareto surface of $D, R$, and $d$ with $P_{\max }=P_{0}$ being the maximum power consumption level of the video encoder.

as Figs. 16(a) and 16(c). This is because that within their D-R models, the encoding time as well as encoding power are always assumed to be fixed but unspecified, which is a special case of the proposed d-P-R-D model. Hence in these work, the standard deviation $\sigma$ of transformed residuals is fixed as a result of fixed $\lambda$ and $\theta$, and their rate control is to tune $Q P$ since $D$ and $R$ are functions of $Q P$ alone. From the D-d curves in Figs. 16(b) and 16(d), it can be seen that $D_{\min }$ decreases with $d_{\max }$ but becomes quite flat for larger $d_{\max }$. This is because that ME has already achieved a global optimal motion vector at a certain value of search range, and hence continuously increasing the search range beyond that value contribute little to the decrement of minimum achievable distortion. 


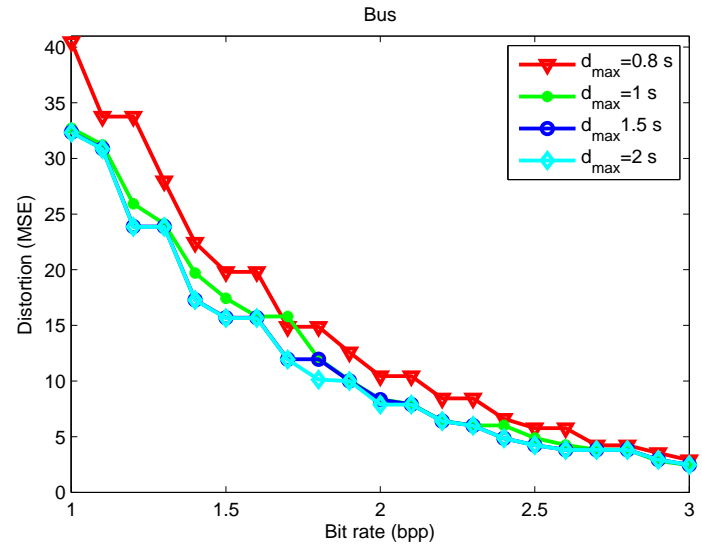

(a)

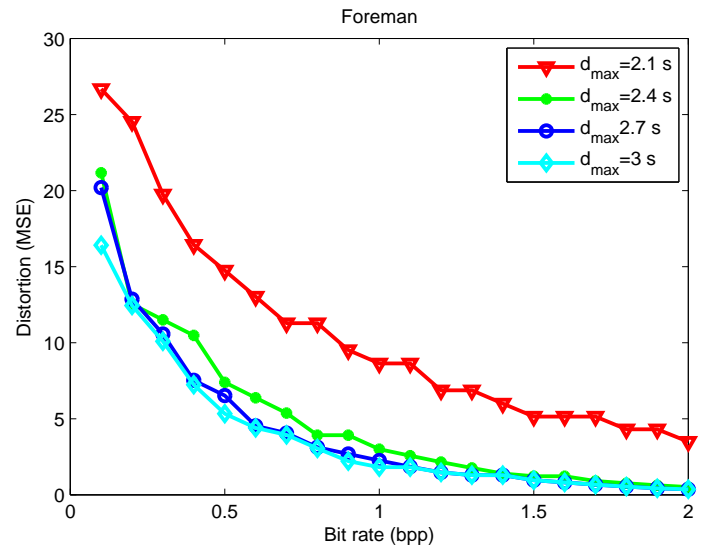

(c)

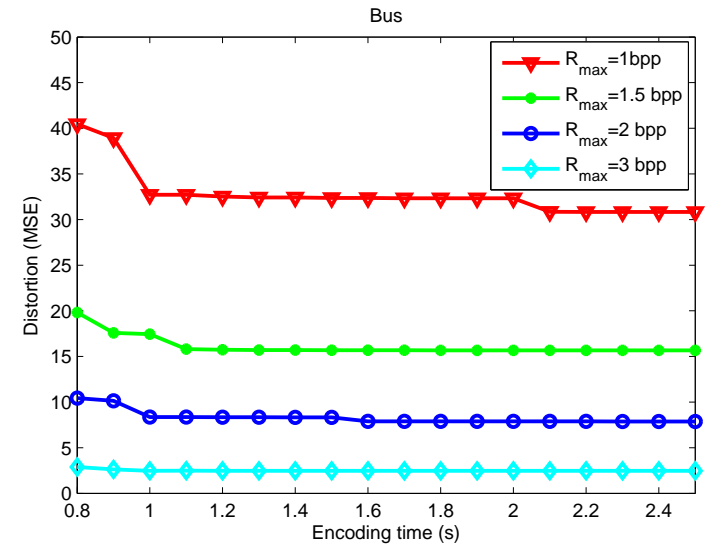

(b)

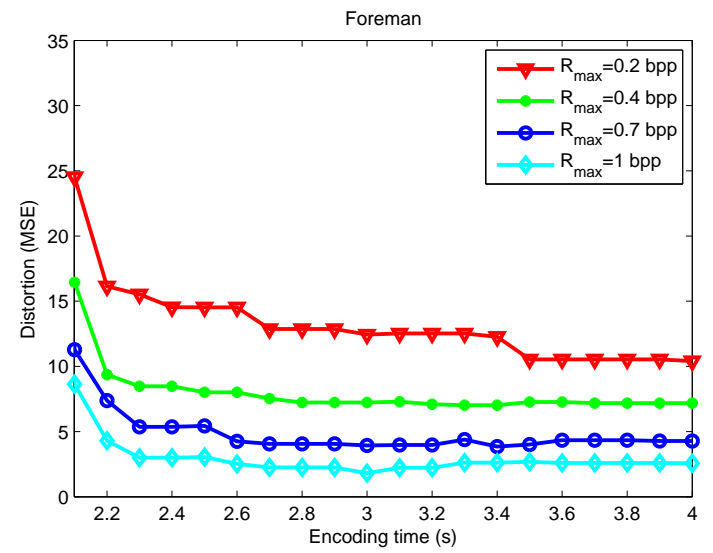

(d)

Fig. 16. D-R curves of (a) Bus sequence and (c) Foreman sequence for different maximum encoding times $d_{\text {max }}$, and D-d curves of (b) Bus sequence and (c) Foreman sequence for different maximum bit rates $R_{\max }$, where $P_{\max }=P_{0}$ is the maximum power consumption level of the video encoder.

Fig. 17 shows the D-R curves for different maximum power consumption levels, and D-P curves for different maximum source bit rates, with the maximum encoding delay fixed at $1 \mathrm{~s}$ and $4 \mathrm{~s}$ for Bus and Foreman sequences. It can be seen from Figs. 17(a) and 17(c) that for a given maximum power consumption level, the relationship of distortion and rate complies with Shannon's source coding theory. As illustrated by the D-P curves in Figs. 17(b) and 17(d), $D_{\min }$ decreases with the increment of the percentage of power consumption but becomes quite flat for larger power consumption level. The reason is same as the previous analysis, i.e., ME has already achieved an global optimal motion vector at a certain value of search range when the power consumption level is relatively large. 


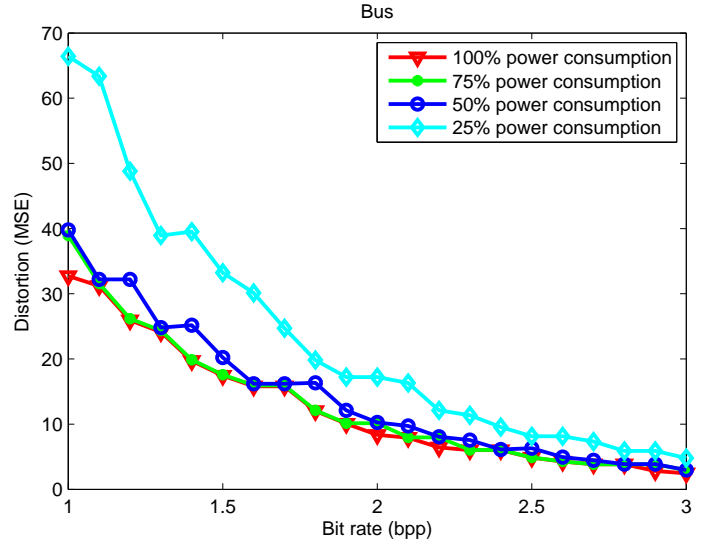

(a)

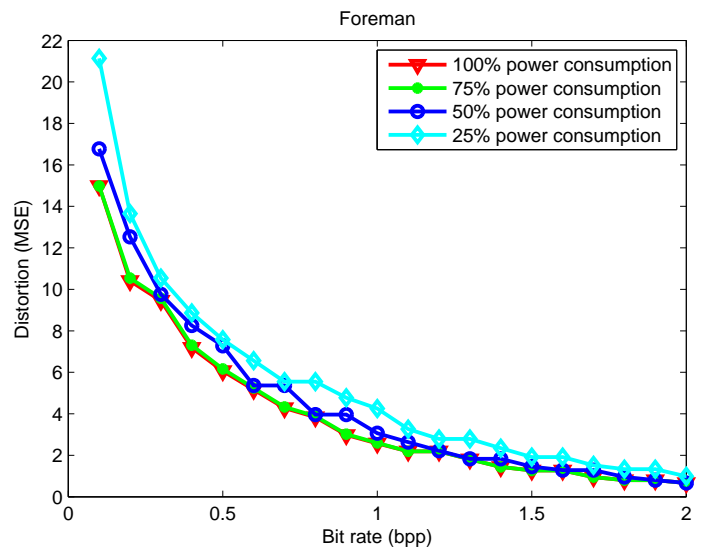

(c)

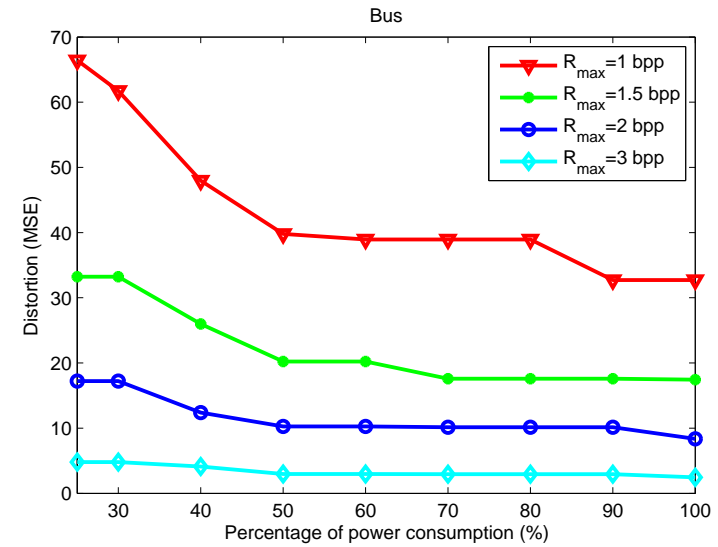

(b)

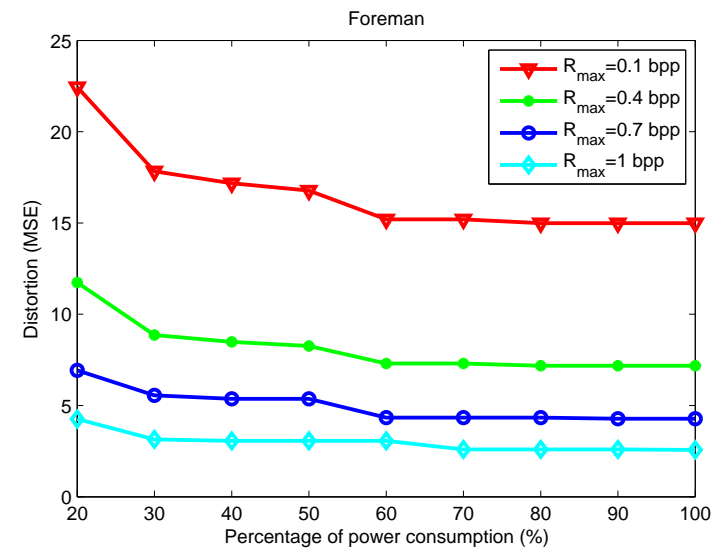

(d)

Fig. 17. D-R curves of (a) Bus sequence and (c) Foreman sequence for different power consumption levels $P_{\max }$, and D-P curves of (b) Bus sequence and (c) Foreman sequence for different maximum bit rates $R_{\max }$, where $d_{\max }$ for Bus sequence and Foreman sequence are set to $1 \mathrm{~s}$ and $4 \mathrm{~s}$, respectively.

Fig. 18 presents the D-d curves for different maximum power consumption levels, and D-P curves for different maximum encoding times, with the maximum source bit rate fixed at 1.5 bpp and 0.5 bpp for Bus and Foreman sequences. It can be seen that the similar analysis of the proposed d-P-R-D model is witnessed as well, i.e., $D_{\min }$ decreases with $d_{\max }$ but becomes quite flat for larger $d_{\max }$, and $D_{\min }$ decreases with the increment of the percentage of power consumption but becomes quite flat for larger power consumption level.

\section{Performance Comparison}

In order to demonstrate the performance of the proposed RC algorithm, the d-P-R-D model is derived from the first 10 frames of a video sequence and the proposed $\mathrm{RC}$ algorithm acts on the 


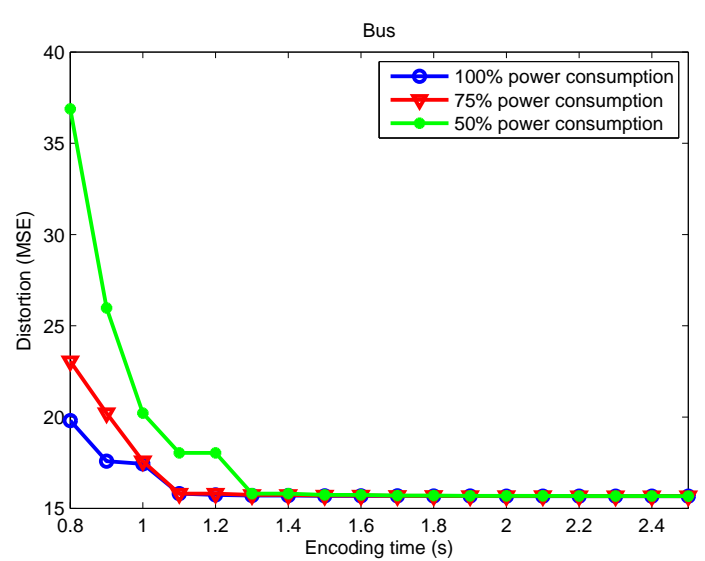

(a)

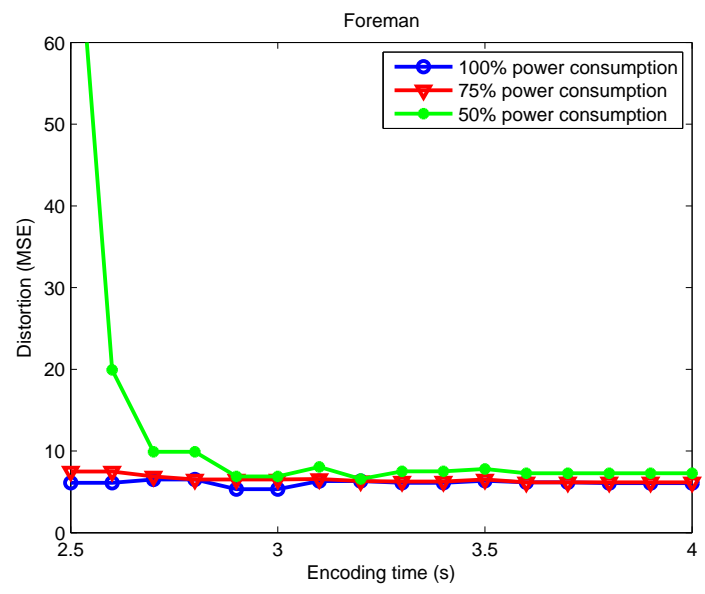

(c)

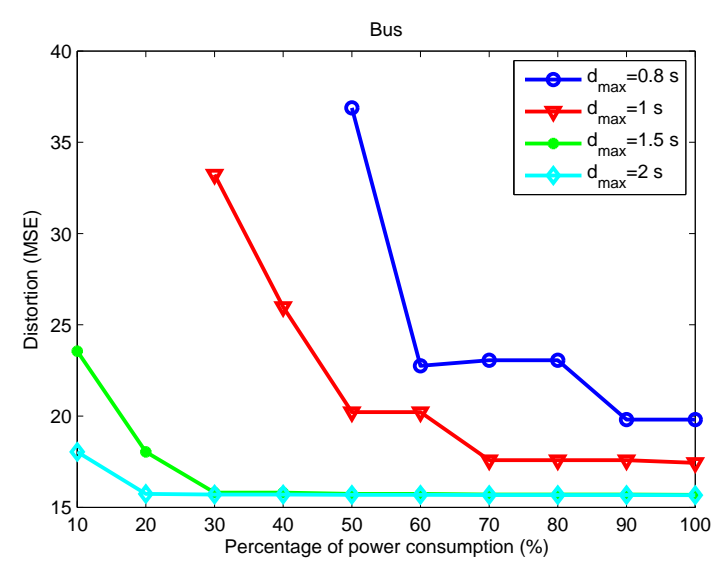

(b)

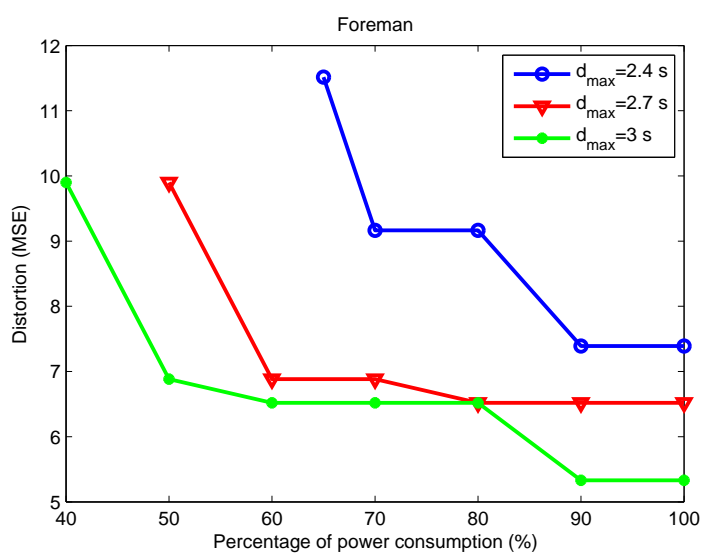

(d)

Fig. 18. D-d curves of (a) Bus sequence and (c) Foreman sequence for different power consumption levels $P_{\max }$, and D-P curves of (b) Bus sequence and (c) Foreman sequence for different maximum encoding times $d_{\max }$, where $R_{\max }$ for Bus sequence and Foreman sequence are set to $1.5 \mathrm{bpp}$ and $0.5 \mathrm{bpp}$, respectively.

entire video sequence. It should be noted that, when a new video scene is acquired, a new model $\sigma(\lambda, Q)=a e^{-b}+c+d Q$ needs to be fitted with three fitting parameters $a, b$ and $c$. In order to have a better fitting result, in this paper, we have used the whole set of the empirical values with different configurations of $\lambda$ and $Q$ to determine the three fitting parameters, which may cause the increase in computational complexity higher. To reduce such complexity in practice, since the function form of $\sigma(\lambda, Q)$ is already known and only three fitting parameters are unknown, we could choose a much smaller subset of empirical values with only a few configurations of $\lambda$ and $Q$ as training set and obtained the standard deviation model with the tradeoff of fitting accuracy. On the other hand, in the proposed RC algorithm, we only need to use the first several P-frames 
(e.g., 10 frames in the experiment) to obtain the standard deviation model and to apply it as an estimated standard deviation model for the whole video sequence (e.g., 300 frames). Therefore, the additional computational complexity introduced by the proposed $\mathrm{RC}$ algorithm per frame is not significant and thus can be neglected.

Fig. 19 and Fig. 20 show the frame-wise objective quality in luminance-component PSNR (Y-PSNR), bit rate, and encoding time with different parameter pairs of $(\lambda, Q P)$ for the first 30 P-frames of Bus, Foreman, Mobile, and Coastguard sequences. For Bus sequence, we set $R_{\max }=100 \mathrm{kbps}, d_{\max }=1 \mathrm{~s}$, and $P_{\max }=P_{0}$. The optimal parameter pair determined by the proposed RC algorithm is $\left(\lambda^{*}, Q P^{*}\right)=(7,41)$. It can be seen that when we vary the value of $Q P$ and fix the search range at the optimal value of 7, even though the overall quality (e.g., setting $(\lambda, Q P)=(7,40))$ may be higher than the optimal Y-PSNR of the proposed RC algorithm, the bit rate constraint has been violated, which indicates that the corresponding solution is not feasible. When $Q P$ is fixed at the optimal value of 41 , on the other hand, setting the search range greater than the optimal value of 7 does not contribute much to improve the coding efficiency, while any search range less than the optimal value would lead to performance degradation. For example, when we set $\lambda$ to the maximum value of 16, the Y-PSNR and bit rate performance is similar to the optimal solution, while the encoding time is much greater than the encoding time constraint. If $\lambda=1$, the Y-PSNR is lower than the optimal solution while the bit rate violates the bit rate constraint. Therefore, the proposed RC algorithm can find the search range threshold over which the quality does not make much improvement, which is particularly useful in saving encoding time and computation power. That is, since the source coding complexity mainly depends on the ME complexity, if we know the coding efficiency at a lower cost (smaller search range) is similar to at a higher cost (larger search range), it is unnecessary to spend the valuable computational resources on extra ME. Thus, the power consumption and computation time can be saved. The similar observation can also be found for Foreman sequence, where $R_{\max }=100 \mathrm{kbps}, d_{\max }=2$ $\mathrm{s}$, and $P_{\max }=P_{0}$, and the optimal parameter pair determined by the proposed RC algorithm is $\left(\lambda^{*}, Q P^{*}\right)=(5,38)$.

In Fig. 21 and Fig. 22, we compare the frame-wise performance of the proposed RC algorithm with the RC scheme in JM18.2 over four test sequences. It should be noted that one of our main contributions is to propose an analytic d-P-R-D model for video encoders and introduce encoding delay and power constraints into traditional source rate control optimization problems, while the 


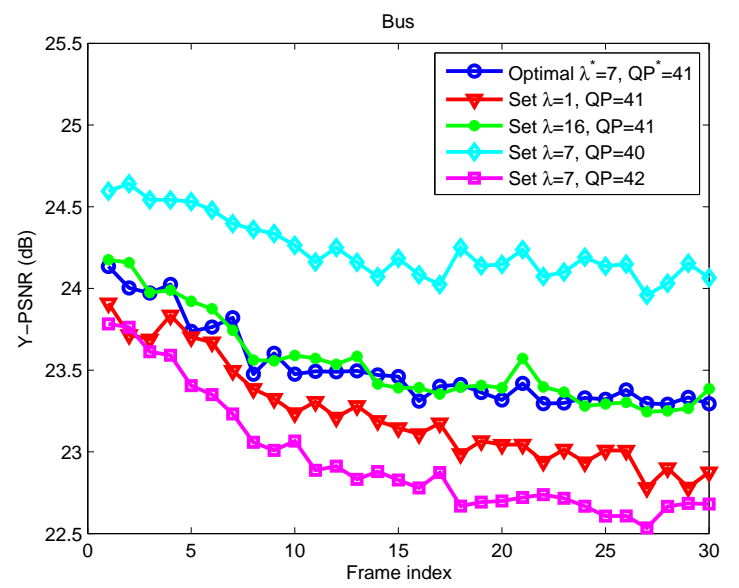

(a)

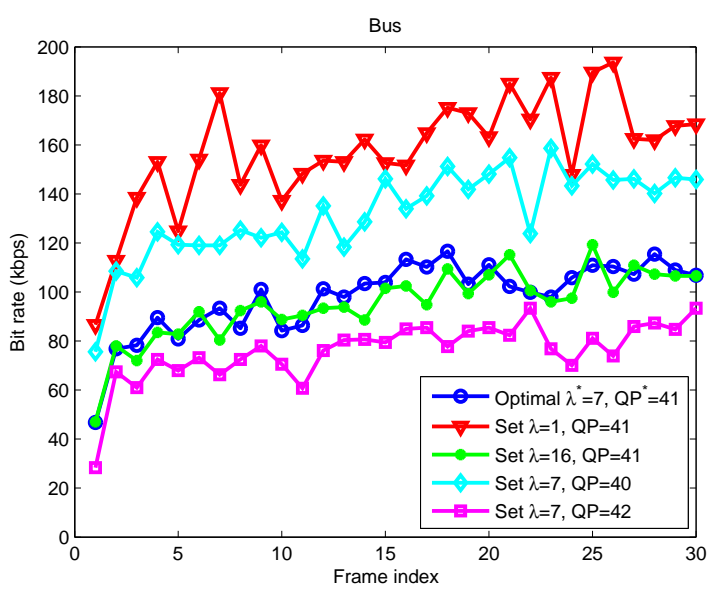

(c)

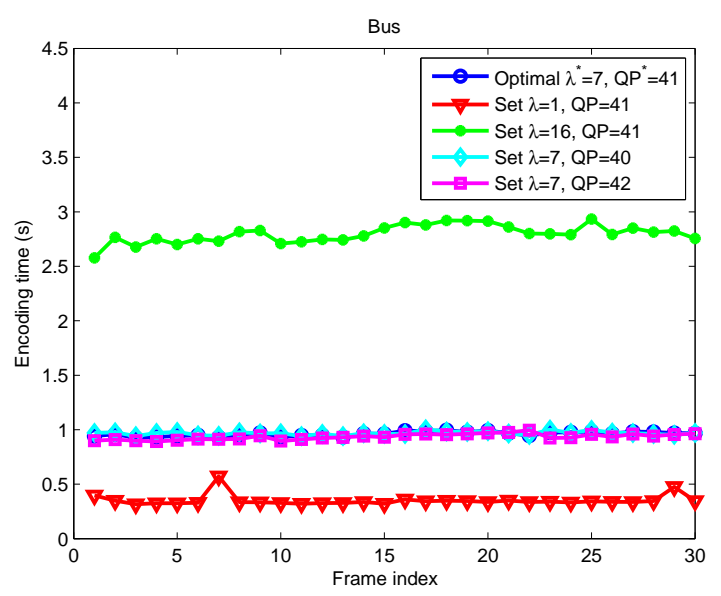

(e)

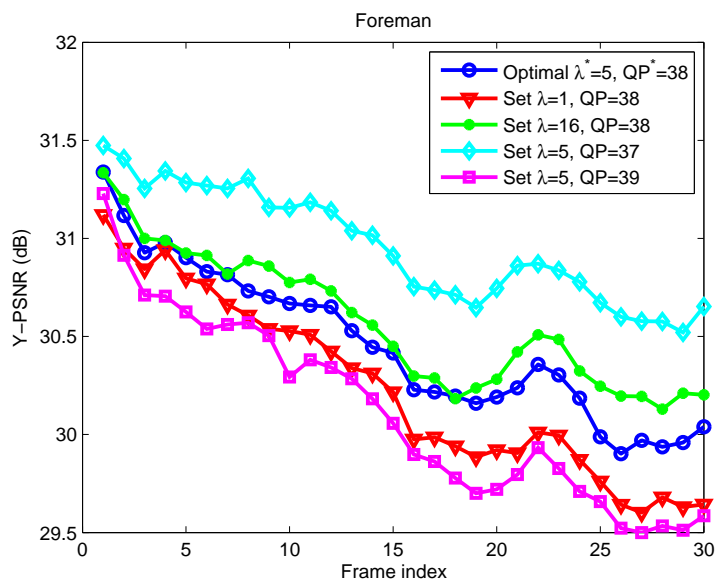

(b)

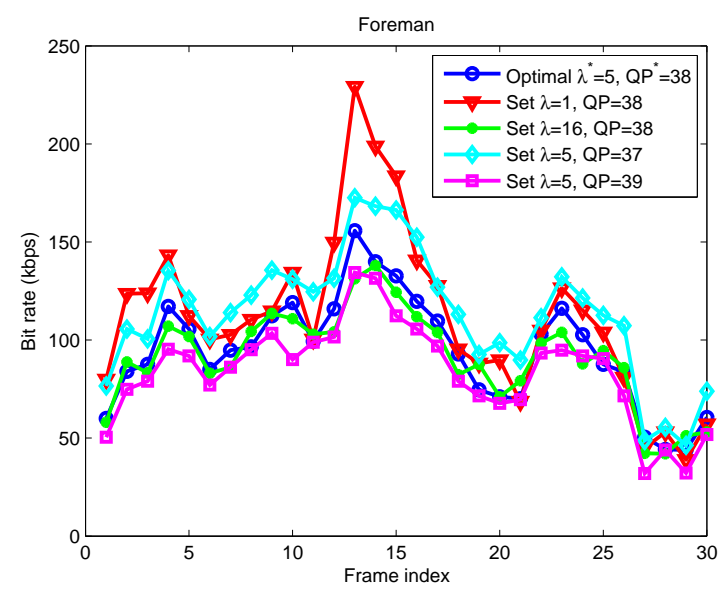

(d)

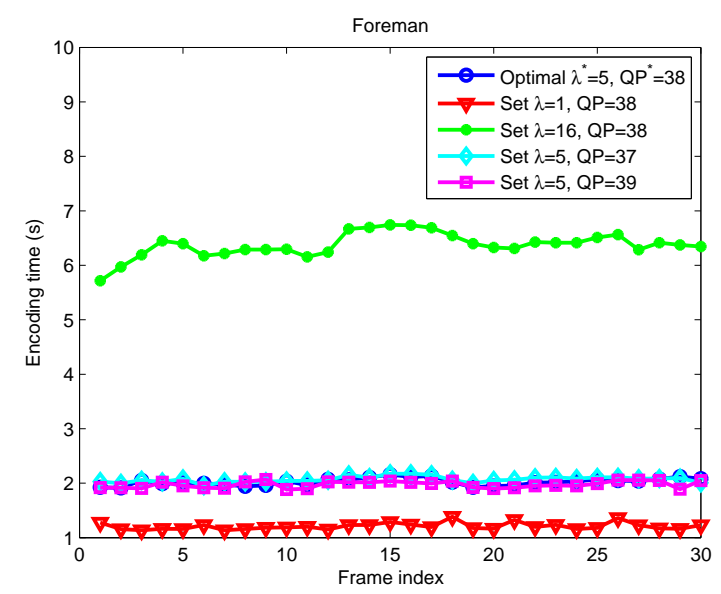

(f)

Fig. 19. (a), (b) The objective quality, (c), (d) bit rate and (e), (f) encoding time with different parameter pairs of $(\lambda, Q P)$ for the first 30 P-frames of Bus and Foreman sequences, where $R_{\max }=100 \mathrm{kbps}, P_{\max }=P_{0}$ is the maximum power consumption level of the video encoder, and $d_{\max }$ is set to $1 \mathrm{~s}$ and $2 \mathrm{~s}$ for Bus and Foreman sequences, respectively. 


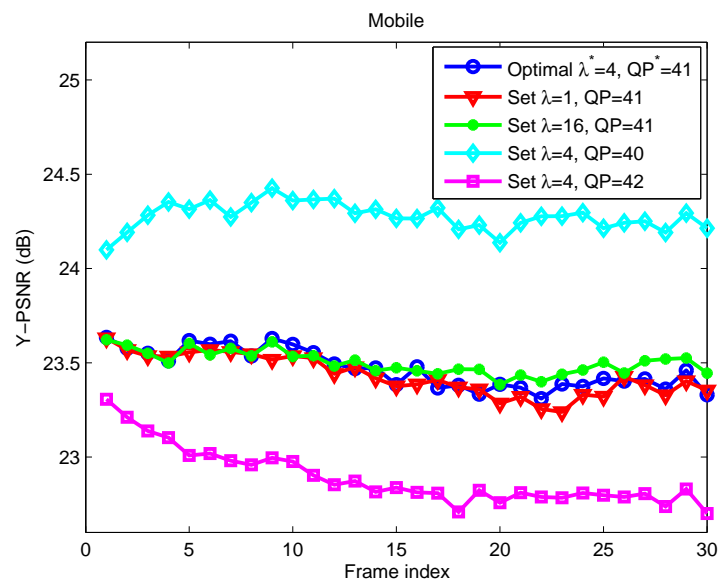

(a)

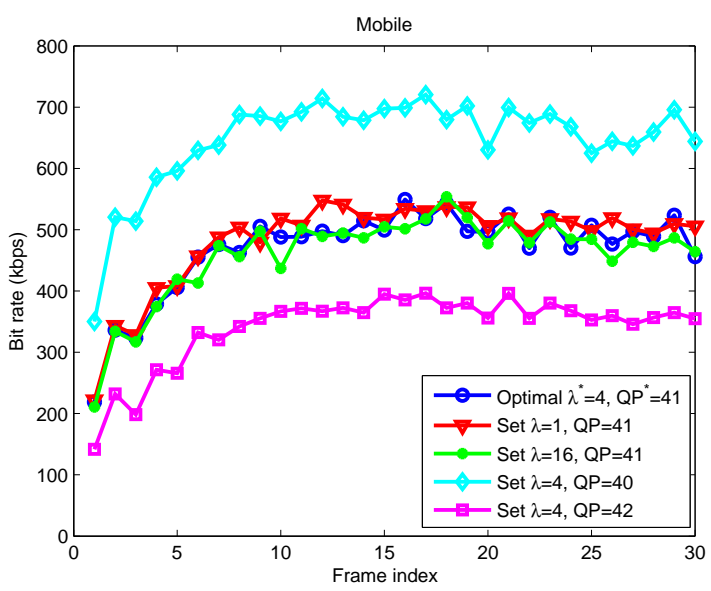

(c)

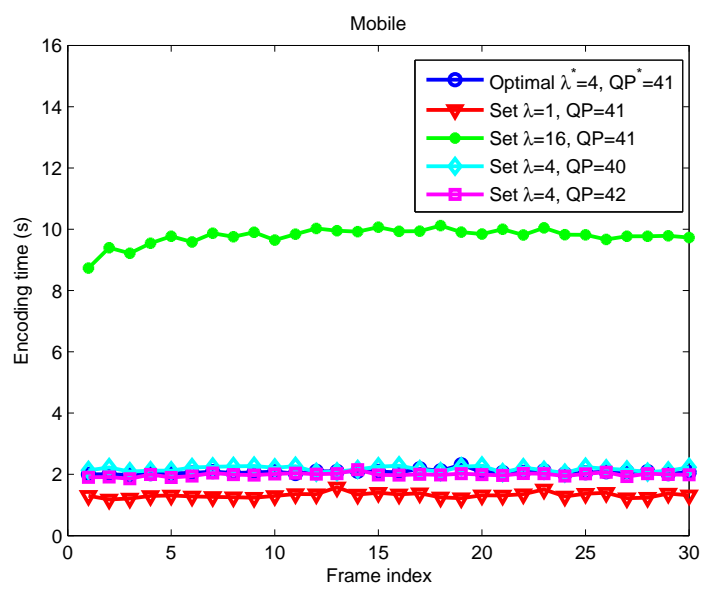

(e)

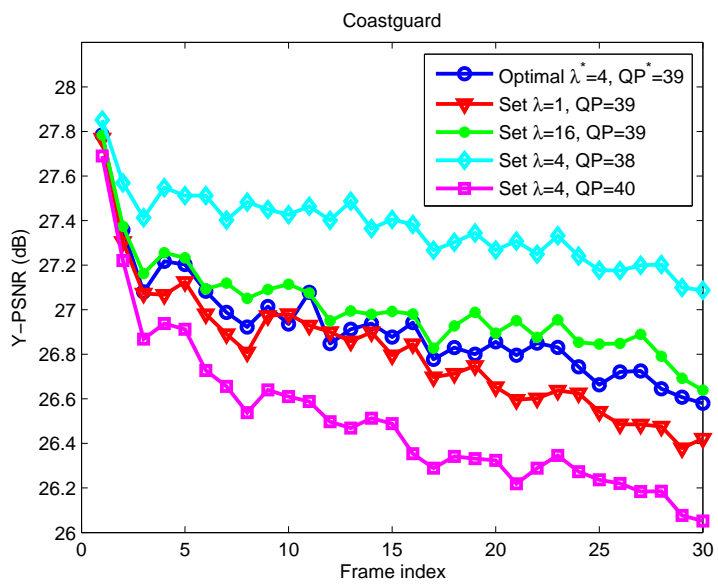

(b)

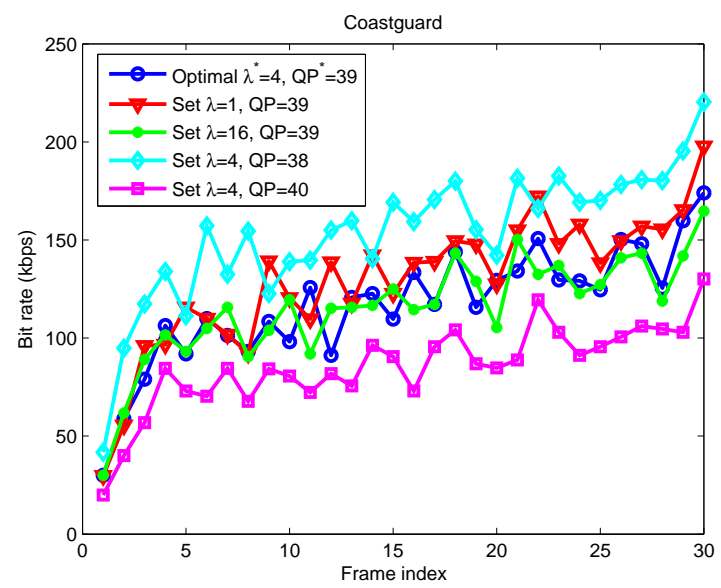

(d)

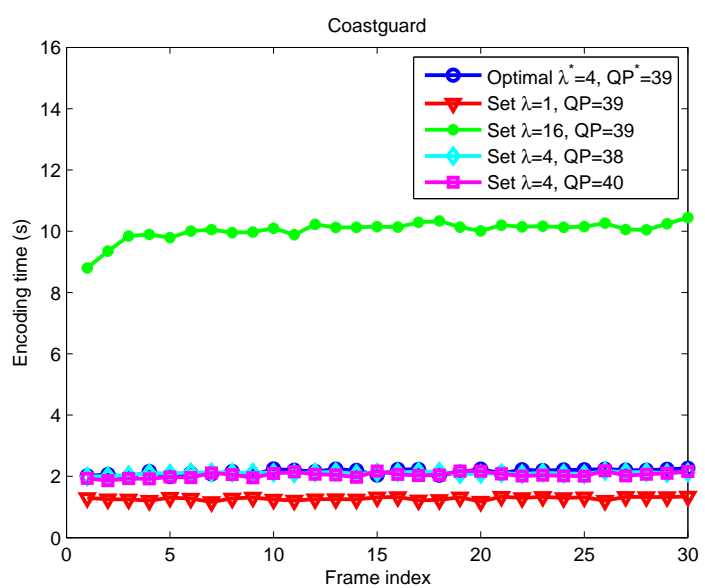

(f)

Fig. 20. (a), (b) The objective quality, (c), (d) bit rate and (e), (f) encoding time with different parameter pairs of $(\lambda, Q P)$ for the first 30 P-frames of Mobile and Coastguard sequences, where $d_{\max }=2 \mathrm{~s}, P_{\max }=P_{0}$ is the maximum power consumption level of the video encoder, and $R_{\max }$ is set to $500 \mathrm{kbps}$ and $100 \mathrm{kbps}$ for Mobile and Coastguard sequences, respectively. 


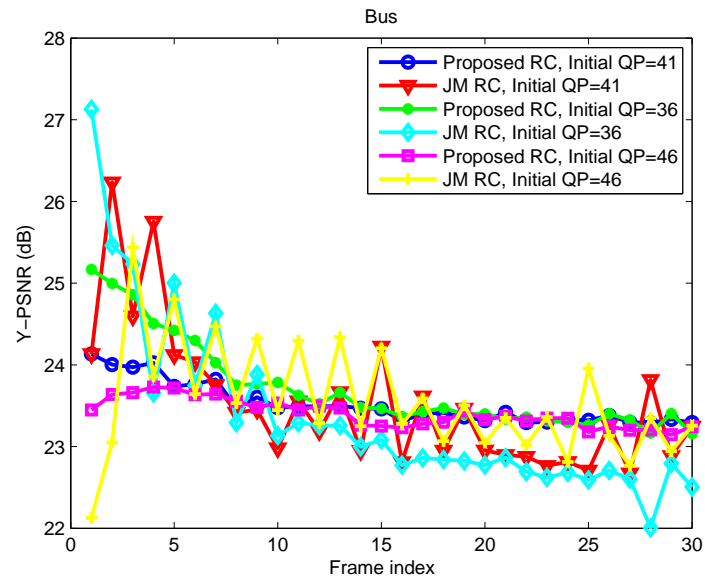

(a)

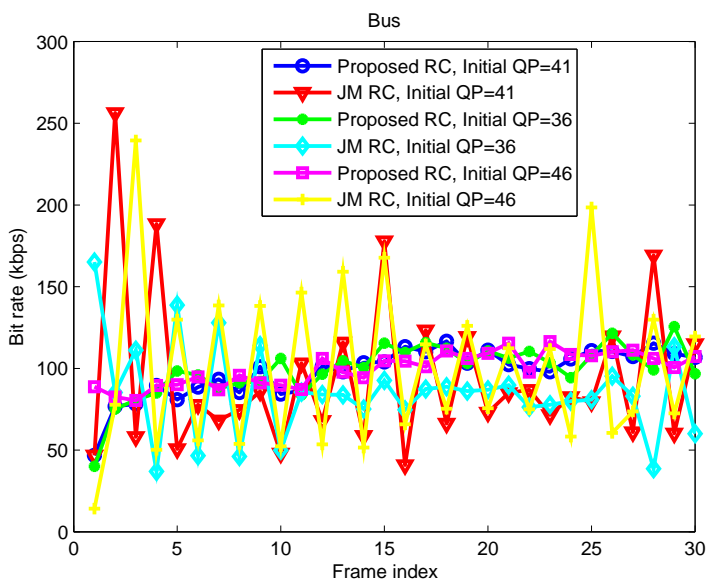

(c)

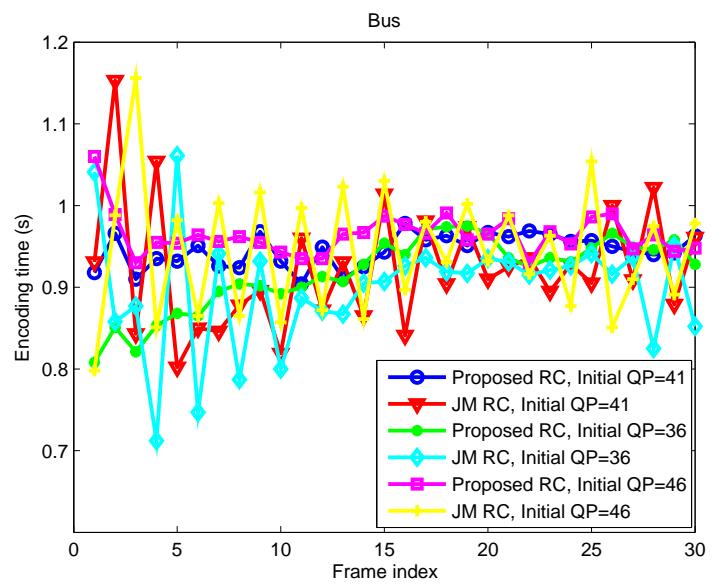

(e)

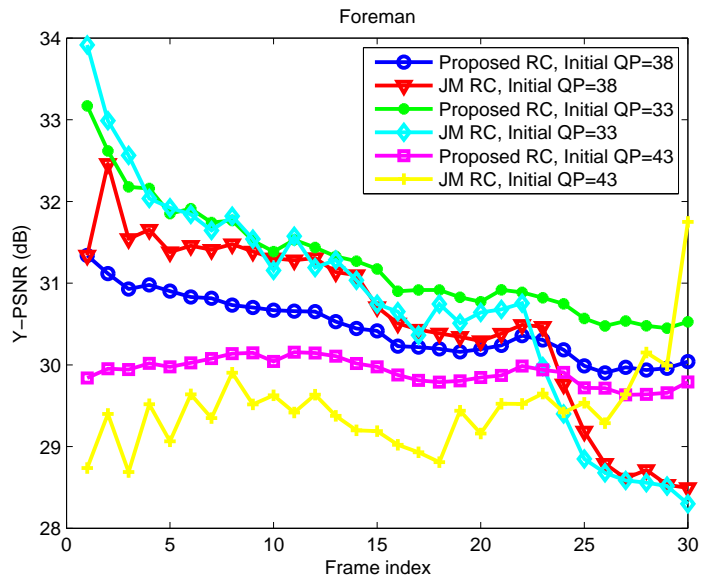

(b)

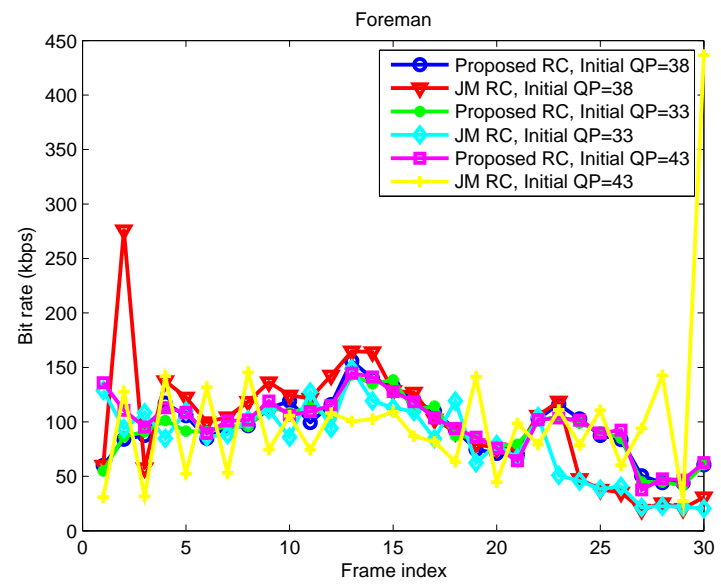

(d)

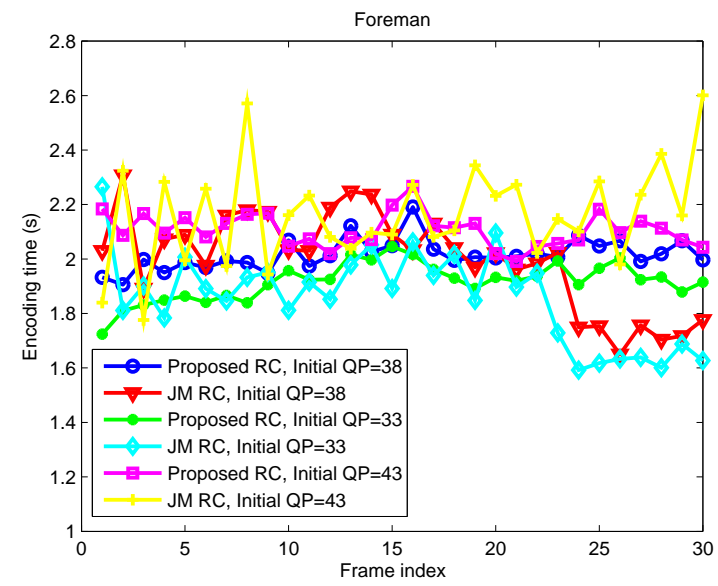

(f)

Fig. 21. (a), (b) objective quality, (c), (d) bit rate and (e), (f) encoding time achieved by the proposed rate control algorithm and the rate control algorithm of JM with different setup of initial $Q P$ for the first I-frame, for the first $30 \mathrm{P}$-frames of Bus and Foreman sequences, where search range is set to the optimal search range solved by the proposed algorithm, $R_{\text {max }}=100$ kbps, $P_{\max }=P_{0}$ is the maximum power consumption level of the video encoder, and $d_{\max }$ is set to $1 \mathrm{~s}$ and $2 \mathrm{~s}$ for Bus and Foreman sequences, respectively. 


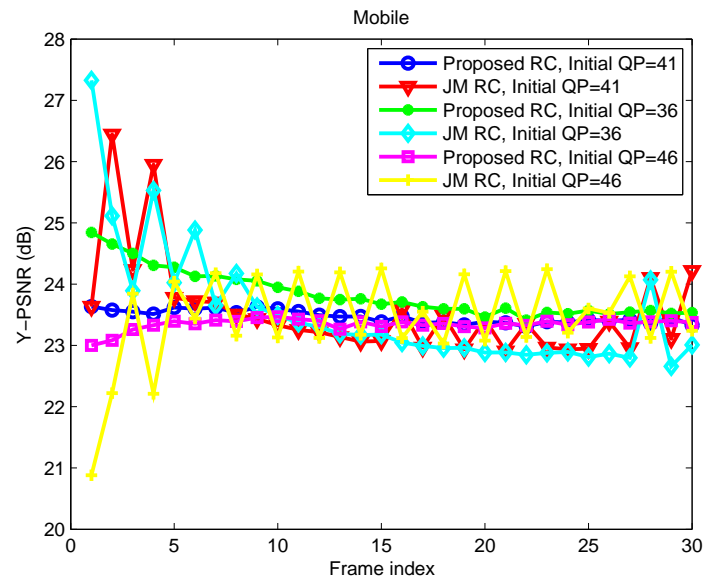

(a)

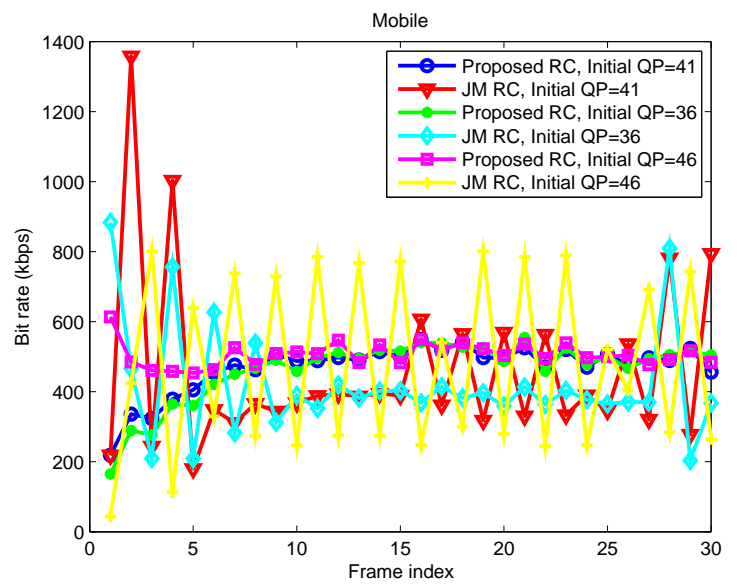

(c)

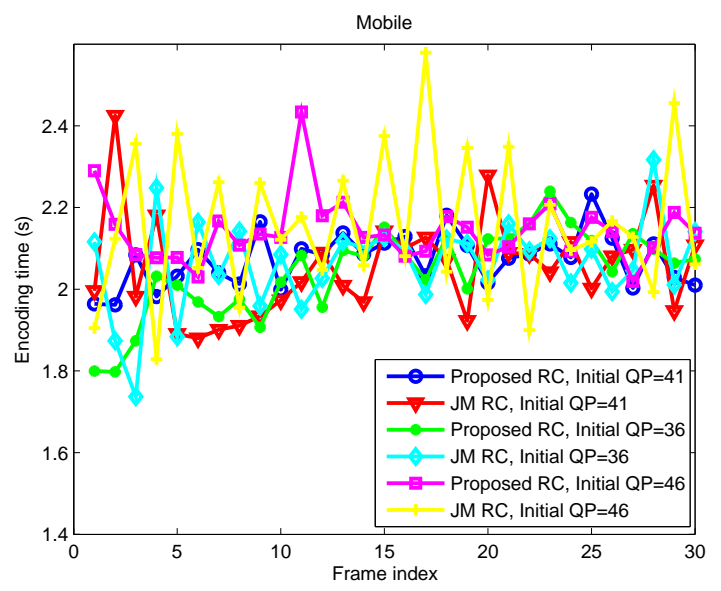

(e)

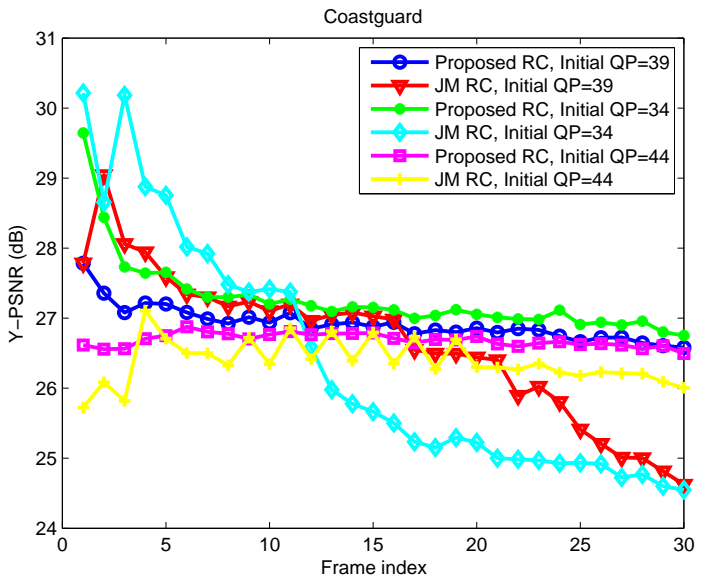

(b)

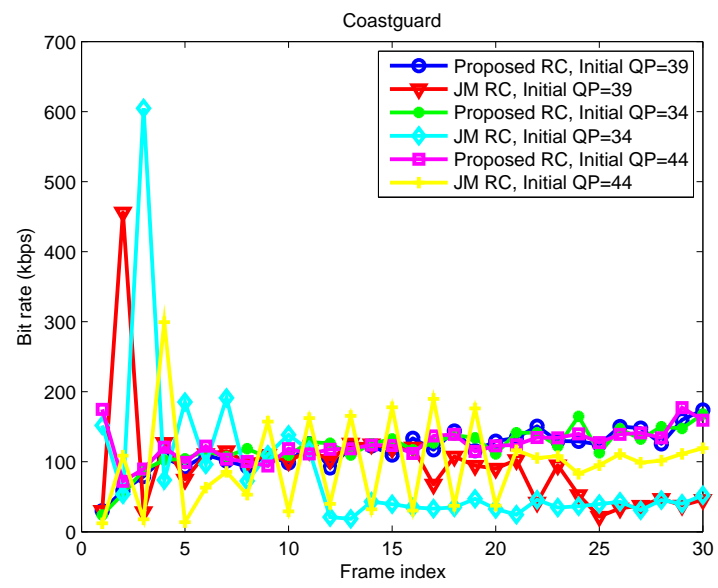

(d)

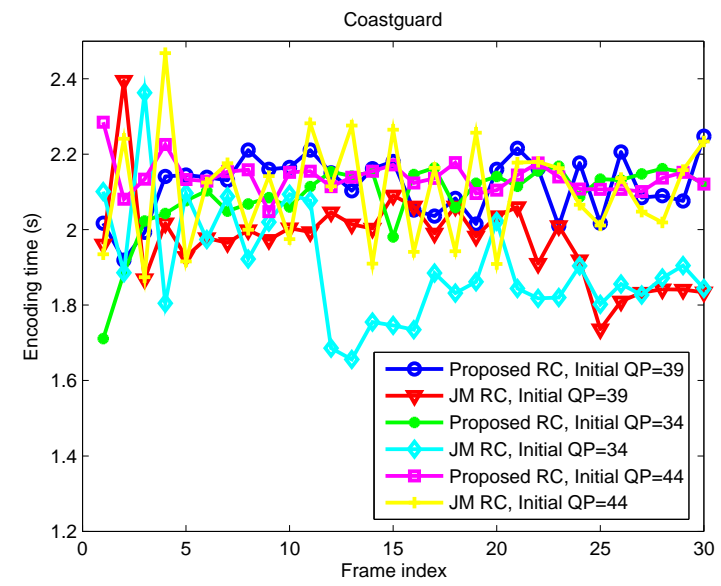

(f)

Fig. 22. (a), (b) objective quality, (c), (d) bit rate and (e), (f) encoding time achieved by the proposed rate control algorithm and the rate control algorithm of JM with different setup of initial $Q P$ for the first I-frame, for the first 30 P-frames of Mobile and Coastguard sequences, where search range is set to the optimal search range solved by the proposed algorithm, $d_{m a x}=2$ $\mathrm{s}, P_{\max }=P_{0}$ is the maximum power consumption level of the video encoder, and $R_{\max }$ is set to $500 \mathrm{kbps}$ and $100 \mathrm{kbps}$ for Mobile and Coastguard sequences, respectively. 
JM RC scheme fails to deal with the delay and power dimensions. In the JM RC scheme, the control unit is a frame, and RDO is utilized based on an R-D model as a function of $Q P$, such that by tuning respective $Q P$ of a frame it can meet the target bit rate while minimizing the coding distortion. However, the delay and power constraints may not be satisfied since the search range is not specified. To meet the maximum delay and power constraints and thus have a "fair" comparison with the proposed RC scheme, here, we set the search range for the JM RC scheme as the optimum value obtained by the proposed RC scheme.

It can be seen that, however, the performance of the JM RC scheme depends greatly on the initial $Q P$ value of the first I-frame. According to [12], the selection of the initial quantization step size for the first I-frame is very critical for model based rate control algorithm, since the ratedistortion of the first frame can affect the coding efficiency of the subsequent frames. Therefore, for JM rate control, if the initial quantization step size is set to be too small, the bit rate for the first few frames would be much larger than the target bit rate. To make the average bit rate meet the target bit rate, the bit rates allocated for the rest few frames would be much smaller by increasing quantization step sizes for these frames. For example, for different setups of initial $Q P$ for the first I-frame, the results of Y-PSNR, bit rate and encoding time per frame obtained by the JM RC scheme are significantly different, while the results of the proposed RC algorithm are relatively similar and not affected too much by different initial $Q P$ values. In addition, the JM RC scheme fails to get a stable performance for the entire sequence which is required for a better user experience. The corresponding Y-PSNR, bit rate, and encoding time vs. frame index curves are quite fluctuated and unstable. Although the Y-PSNR values for some frames might be higher than those of the proposed RC algorithm, the bit rate and encoding time constraints are violated. In contrast, the selection of the initial quantization step size for the first I-frame would have less impact on the performance of the proposed RC algorithm. The proposed RC algorithm can achieve smooth and stable Y-PSNR performance while the other constraints are satisfied.

The reason for the stability of the proposed $\mathrm{RC}$ algorithm is as follows. For each video sequence, specifically, the proposed d-P-R-D model is derived based on the first several frames and thus can characterize more accurately the statistics in that video sequence. When adopting such d-P-R-D model in the proposed model based rate control algorithm, the optimal coding parameters can be determined in accordance with different video sequences. And for each 
TABLE I

COMPARISON OF AVERAGE Y-PSNR, BIT RATE AND ENCODING TIME (ET) UNDER DIFFERENT CONSTRAINTS OF MAXIMUM POWER CONSUMPTION LEVELS, BIT RATE, ENCODING DELAY, AND DIFFERENT SETUP IN THE INITIAL $Q P$ FOR THE FIRST I-FRAME, FOR THE FIRST 150 P-FRAMES OF Bus SEQUENCE.

\begin{tabular}{|c|c|c|c|c|c|c|c|c|c|}
\hline \multirow{2}{*}{\multicolumn{2}{|c|}{$\frac{P_{\max }(\%)}{d_{\max }(\mathrm{s})}$}} & \multicolumn{4}{|c|}{100} & \multicolumn{4}{|c|}{50} \\
\hline & & \multicolumn{2}{|c|}{0.5} & \multicolumn{2}{|c|}{1} & \multicolumn{2}{|c|}{0.5} & \multicolumn{2}{|c|}{1} \\
\hline \multicolumn{2}{|c|}{$R_{\max }(\mathrm{kbps})$} & 100 & 500 & 100 & 500 & 100 & 500 & 100 & 500 \\
\hline \multirow{3}{*}{$\begin{array}{c}\text { Proposed } \\
\text { RC } \\
Q P^{*} \\
\end{array}$} & Y-PSNR(dB) & 23.38 & 29.93 & 23.56 & 30.79 & 22.58 & 29.10 & 23.51 & 30.77 \\
\hline & Rate(kbps) & 109.16 & 515.61 & 101.69 & 536.99 & 100.60 & 520.84 & 103.34 & 542.45 \\
\hline & ET(s) & 0.49 & 0.45 & 0.95 & 1.10 & 0.51 & 0.49 & 1.01 & 0.99 \\
\hline \multirow{3}{*}{$\begin{array}{c}\mathrm{JM} \\
\mathrm{RC} \\
Q P^{*}\end{array}$} & Y-PSNR(dB) & 23.14 & 29.68 & 23.59 & 30.28 & 22.52 & 28.86 & 23.48 & 30.22 \\
\hline & Rate(kbps) & 98.89 & 498.37 & 99.14 & 498.27 & 99.07 & 499.37 & 98.95 & 497.99 \\
\hline & ET(s) & 0.49 & 0.44 & 0.94 & 1.07 & 0.52 & 0.49 & 0.99 & 0.96 \\
\hline \multirow{3}{*}{$\begin{array}{c}\text { Proposed } \\
\text { RC } \\
Q P^{*}-10 \\
\end{array}$} & Y-PSNR(dB) & 23.44 & 29.95 & 23.63 & 30.84 & 22.56 & 29.12 & 23.57 & 30.82 \\
\hline & Rate(kbps) & 110.61 & 515.92 & 102.58 & 536.93 & 101.99 & 516.42 & 103.16 & 541.58 \\
\hline & ET(s) & 0.49 & 0.46 & 0.93 & 1.09 & 0.51 & 0.47 & 1.01 & 0.97 \\
\hline \multirow{3}{*}{$\begin{array}{c}\mathrm{JM} \\
\mathrm{RC} \\
Q P^{*}-10\end{array}$} & Y-PSNR(dB) & 22.87 & 29.54 & 23.39 & 30.16 & 22.24 & 28.76 & 23.33 & 30.12 \\
\hline & Rate(kbps) & 95.63 & 494.30 & 96.21 & 493.51 & 96.18 & 495.14 & 95.72 & 492.87 \\
\hline & ET(s) & 0.49 & 0.46 & 0.91 & 1.09 & 0.51 & 0.48 & 0.99 & 0.95 \\
\hline \multirow{3}{*}{$\begin{array}{l}\text { Proposed } \\
\text { RC } \\
Q P^{*}+10\end{array}$} & Y-PSNR(dB) & 23.44 & 29.92 & 23.53 & 30.81 & 22.58 & 29.12 & 23.52 & 30.80 \\
\hline & Rate(kbps) & 112.45 & 517.22 & 104.59 & 540.01 & 102.97 & 518.41 & 105.55 & 543.74 \\
\hline & $\mathrm{ET}(\mathrm{s})$ & 0.50 & 0.47 & 0.94 & 1.11 & 0.51 & 0.50 & 1.01 & 0.99 \\
\hline \multirow{3}{*}{$\begin{array}{c}\mathrm{JM} \\
\mathrm{RC} \\
Q P^{*}+10\end{array}$} & Y-PSNR(dB) & 22.75 & 29.60 & 23.15 & 30.22 & 22.21 & 28.75 & 23.07 & 30.16 \\
\hline & Rate(kbps) & 100.53 & 502.33 & 100.39 & 501.89 & 100.50 & 502.34 & 100.75 & 502.08 \\
\hline & ET(s) & 0.48 & 0.46 & 0.92 & 1.08 & 0.52 & 0.49 & 1.00 & 0.96 \\
\hline
\end{tabular}

sequence, $\lambda^{*}$ and $Q^{*}$ would remain the same for all frames, which makes the actual bit rate and Y-PSNR more stable among different frames. For JM RC scheme, the quantization step size is tuned and varied for each frame in accordance with general distortion-quantization (D-Q) and rate-quantization (R-Q) models which are independent of different video contents. Therefore, the optimal quantization step size determined by JM RC scheme would change frame by frame in order to meet the target bit rate while minimizing the coding distortion. And the actual bit rate and Y-PSNR would fluctuate with such change in quantization step size among different frames.

Tables I-IV show the comparison of average Y-PSNR, bit rate, and encoding time for the first 150 P-frames of Bus, Foreman, Mobile, and Coastguard sequences, respectively. The maximum achieved Y-PSNR results can verify the analysis of the d-P-R-D model in Sec. IV-B. It can also be seen that the selection of the initial quantization step size for the first I-frame would have 
TABLE II

COMPARISON OF AVERAGE Y-PSNR, BIT RATE AND ENCODING TIME (ET) UNDER DIFFERENT CONSTRAINTS OF MAXIMUM POWER CONSUMPTION LEVELS, BIT RATE, ENCODING DELAY, AND DIFFERENT SETUP IN THE INITIAL QP FOR THE FIRST I-FRAME, FOR THE FIRST 150 P-FRAMES OF Foreman SEQUENCE.

\begin{tabular}{|c|c|c|c|c|c|c|c|c|c|}
\hline \multirow{2}{*}{\multicolumn{2}{|c|}{$\frac{P_{\max }(\%)}{d_{\max }(\mathrm{s})}$}} & \multicolumn{4}{|c|}{100} & \multicolumn{4}{|c|}{50} \\
\hline & & \multicolumn{2}{|c|}{2} & \multicolumn{2}{|c|}{3} & \multicolumn{2}{|c|}{2} & \multicolumn{2}{|c|}{3} \\
\hline \multicolumn{2}{|c|}{$R_{\max }(\mathrm{kbps})$} & 100 & 500 & 100 & 500 & 100 & 500 & 100 & 500 \\
\hline \multirow{3}{*}{$\begin{array}{c}\text { Proposed } \\
\text { RC } \\
Q P^{*} \\
\end{array}$} & Y-PSNR(dB) & 30.28 & 35.98 & 30.41 & 36.01 & 29.65 & 35.92 & 30.31 & 36.00 \\
\hline & Rate(kbps) & 105.70 & 543.85 & 103.68 & 538.24 & 96.79 & 564.10 & 104.77 & 542.50 \\
\hline & ET(s) & 2.14 & 2.13 & 3.12 & 2.85 & 2.03 & 1.98 & 3.11 & 3.08 \\
\hline \multirow{3}{*}{$\begin{array}{c}\mathrm{JM} \\
\mathrm{RC} \\
Q P^{*}\end{array}$} & Y-PSNR(dB) & 30.04 & 35.67 & 30.27 & 35.73 & 29.68 & 35.49 & 30.17 & 35.71 \\
\hline & Rate(kbps) & 100.31 & 494.69 & 102.02 & 494.46 & 99.10 & 494.40 & 100.92 & 494.73 \\
\hline & ET(s) & 2.06 & 2.12 & 3.06 & 2.87 & 2.05 & 1.88 & 3.07 & 3.12 \\
\hline \multirow{3}{*}{$\begin{array}{c}\text { Proposed } \\
\text { RC } \\
Q P^{*}-10 \\
\end{array}$} & Y-PSNR(dB) & 30.72 & 36.09 & 30.81 & 36.13 & 30.08 & 36.05 & 30.74 & 36.12 \\
\hline & Rate(kbps) & 106.56 & 544.56 & 104.01 & 539.72 & 96.79 & 563.47 & 105.00 & 541.28 \\
\hline & ET(s) & 2.10 & 2.20 & 3.03 & 2.82 & 1.94 & 1.88 & 2.96 & 3.17 \\
\hline \multirow{3}{*}{$\begin{array}{c}\mathrm{JM} \\
\mathrm{RC} \\
Q P^{*}-10\end{array}$} & Y-PSNR(dB) & 30.14 & 35.50 & 30.30 & 35.54 & 29.82 & 35.26 & 30.27 & 35.52 \\
\hline & Rate(kbps) & 96.85 & 470.75 & 95.43 & 472.71 & 95.38 & 471.16 & 97.16 & 472.58 \\
\hline & ET(s) & 2.00 & 2.13 & 2.96 & 2.82 & 2.03 & 1.97 & 2.89 & 3.06 \\
\hline \multirow{3}{*}{$\begin{array}{l}\text { Proposed } \\
\text { RC } \\
Q P^{*}+10\end{array}$} & Y-PSNR(dB) & 30.02 & 35.94 & 30.14 & 35.96 & 29.35 & 35.89 & 30.05 & 35.95 \\
\hline & Rate(kbps) & 109.09 & 550.55 & 107.88 & 546.15 & 99.91 & 569.65 & 108.34 & 549.28 \\
\hline & $\mathrm{ET}(\mathrm{s})$ & 2.13 & 2.10 & 3.15 & 2.84 & 2.01 & 2.02 & 3.24 & 3.08 \\
\hline \multirow{3}{*}{$\begin{array}{c}\mathrm{JM} \\
\mathrm{RC} \\
Q P^{*}+10\end{array}$} & Y-PSNR(dB) & 29.83 & 35.61 & 29.93 & 35.67 & 29.45 & 35.42 & 29.85 & 35.64 \\
\hline & Rate(kbps) & 103.49 & 502.03 & 101.69 & 501.55 & 102.84 & 501.23 & 101.52 & 502.01 \\
\hline & ET(s) & 2.10 & 2.07 & 3.05 & 2.86 & 1.94 & 1.99 & 3.02 & 3.15 \\
\hline
\end{tabular}

less impact on the average Y-PSNR of the proposed RC scheme. That is, for the proposed RC scheme, the average Y-PSNR performance under different selection of the initial quantization step size is stable and usually better than that of the JM RC scheme. Although the mismatch of the rate and encoding time may be a little larger at some times, most of them are still within a certain tolerance region, say $10 \%$, and the source rate and encoding time constraints can be still considered as satisfied in practice.

\section{CONCLUSION AND Future WORK}

In this paper, we derived the analytic delay-power-rate-distortion (d-P-R-D) model for IPPPP coding mode in H.264/AVC to investigate the relationship among video encoding time, power, rate and distortion. On the basis of the proposed d-P-R-D model, a model-based source rate control problem has been formulated to minimize the encoding distortion under the constraints 
TABLE III

COMPARISON OF AVERAGE Y-PSNR, BIT RATE AND ENCODING TIME (ET) UNDER DIFFERENT CONSTRAINTS OF MAXIMUM POWER CONSUMPTION LEVELS, BIT RATE, ENCODING DELAY, AND DIFFERENT SETUP IN THE INITIAL QP FOR THE FIRST I-FRAME, FOR THE FIRST 150 P-FRAMES OF Mobile SEQUENCE.

\begin{tabular}{|c|c|c|c|c|c|c|c|c|c|}
\hline \multirow{2}{*}{\multicolumn{2}{|c|}{$\frac{P_{\max }(\%)}{d_{\max }(\mathrm{s})}$}} & \multicolumn{4}{|c|}{100} & \multicolumn{4}{|c|}{50} \\
\hline & & \multicolumn{2}{|c|}{2} & \multicolumn{2}{|c|}{3} & \multicolumn{2}{|c|}{2} & \multicolumn{2}{|c|}{3} \\
\hline \multicolumn{2}{|c|}{$R_{\max }(\mathrm{kbps})$} & 500 & 1000 & 500 & 1000 & 500 & 1000 & 500 & 1000 \\
\hline \multirow{3}{*}{$\begin{array}{c}\text { Proposed } \\
\text { RC } \\
Q P^{*}\end{array}$} & Y-PSNR(dB) & 23.05 & 25.56 & 23.08 & 25.58 & 23.02 & 25.54 & 23.08 & 25.58 \\
\hline & Rate(kbps) & 488.58 & 1027.9 & 486.23 & 1019.7 & 495.78 & 1028.8 & 486.63 & 1021.4 \\
\hline & ET(s) & 2.34 & 2.08 & 3.03 & 3.27 & 2.01 & 2.14 & 3.24 & 2.89 \\
\hline \multirow{3}{*}{$\begin{array}{c}\mathrm{JM} \\
\mathrm{RC} \\
Q P^{*}\end{array}$} & Y-PSNR(dB) & 23.18 & 25.43 & 23.21 & 25.48 & 23.16 & 25.40 & 23.19 & 25.48 \\
\hline & Rate(kbps) & 491.42 & 989.76 & 491.50 & 993.02 & 491.69 & 993.06 & 492.41 & 992.26 \\
\hline & ET(s) & 2.26 & 2.06 & 3.06 & 3.33 & 2.01 & 2.11 & 3.20 & 2.94 \\
\hline \multirow{3}{*}{$\begin{array}{c}\text { Proposed } \\
\text { RC } \\
Q P^{*}-10\end{array}$} & Y-PSNR(dB) & 23.18 & 25.60 & 23.21 & 25.63 & 23.15 & 25.58 & 23.19 & 25.62 \\
\hline & Rate(kbps) & 488.16 & 1022.6 & 485.11 & 1016.9 & 495.05 & 1024.6 & 487.59 & 1017.3 \\
\hline & ET(s) & 2.29 & 1.88 & 3.02 & 3.12 & 1.86 & 2.12 & 3.25 & 2.78 \\
\hline \multirow{3}{*}{$\begin{array}{c}\mathrm{JM} \\
\mathrm{RC} \\
Q P^{*}-10\end{array}$} & Y-PSNR(dB) & 23.02 & 25.26 & 23.08 & 25.31 & 22.97 & 25.23 & 23.06 & 25.28 \\
\hline & Rate(kbps) & 469.37 & 963.60 & 470.01 & 966.30 & 471.36 & 964.63 & 471.10 & 966.16 \\
\hline & $\mathrm{ET}(\mathrm{s})$ & 2.06 & 1.92 & 3.06 & 3.31 & 2.02 & 1.99 & 3.16 & 2.95 \\
\hline \multirow{3}{*}{$\begin{array}{c}\text { Proposed } \\
\text { RC } \\
Q P^{*}+10\end{array}$} & Y-PSNR(dB) & 23.08 & 25.54 & 23.08 & 25.58 & 23.03 & 25.52 & 23.08 & 25.56 \\
\hline & Rate(kbps) & 502.25 & 1036.3 & 498.76 & 1029.7 & 507.71 & 1038.7 & 501.50 & 1035.7 \\
\hline & $\mathrm{ET}(\mathrm{s})$ & 2.17 & 1.88 & 3.04 & 3.27 & 1.93 & 2.14 & 3.20 & 2.91 \\
\hline \multirow{3}{*}{$\begin{array}{c}\mathrm{JM} \\
\mathrm{RC} \\
Q P^{*}+10\end{array}$} & Y-PSNR $(\mathrm{dB})$ & 22.96 & 25.37 & 22.98 & 25.44 & 22.90 & 25.34 & 22.97 & 25.42 \\
\hline & Rate(kbps) & 503.29 & 1002.8 & 501.76 & 1002.0 & 502.05 & 1001.9 & 501.43 & 1001.3 \\
\hline & ET(s) & 2.13 & 1.93 & 3.07 & 3.27 & 1.89 & 2.03 & 3.27 & 2.93 \\
\hline
\end{tabular}

of rate, delay and power. To solve the rate control problem, we proposed a practical algorithm to iteratively update the primal and dual variables by utilizing both the KKT conditions and the SQP method. Experimental results have verified the accuracy of the proposed d-P-R-D model and demonstrated the optimization performance of the model-based source rate control algorithm. The d-P-R-D model and the model based rate control provided a theoretical basis and a practical guideline for the cross-layer system design and performance optimization in wireless video communication under delay and energy constraints. To further tackle the issue of bandwidth fluctuations and higher packet losses in wireless transmissions, our future work will focus on applying the proposed d-P-R-D approach to joint resource allocation and control for the entire wireless video communication system, which aims at minimizing the end-to-end distortion under the constraints of the transmission bandwidth, the end-to-end delay, and the total available power supply of the wireless video communication system. 
TABLE IV

COMPARISON OF AVERAGE Y-PSNR, BIT RATE, AND ENCODING TIME (ET) UNDER DIFFERENT CONSTRAINTS OF MAXIMUM POWER CONSUMPTION LEVELS, BIT RATE, ENCODING DELAY, AND DIFFERENT SETUP OF THE INITIAL QP IN THE FIRST I-FRAME, FOR THE FIRST 150 P-FRAMES OF Coastguard SEQUENCE.

\begin{tabular}{|c|c|c|c|c|c|c|c|c|c|}
\hline \multirow{2}{*}{\multicolumn{2}{|c|}{$\frac{P_{\max }(\%)}{d_{\max }(\mathrm{s})}$}} & \multicolumn{4}{|c|}{100} & \multicolumn{4}{|c|}{50} \\
\hline & & \multicolumn{2}{|c|}{2} & \multicolumn{2}{|c|}{3} & \multicolumn{2}{|c|}{2} & \multicolumn{2}{|c|}{3} \\
\hline \multicolumn{2}{|c|}{$R_{\max }(\mathrm{kbps})$} & 100 & 500 & 100 & 500 & 100 & 500 & 100 & 500 \\
\hline \multirow{3}{*}{$\begin{array}{c}\text { Proposed } \\
\text { RC } \\
Q P^{*}\end{array}$} & Y-PSNR(dB) & 24.88 & 29.09 & 24.97 & 29.15 & 24.82 & 29.04 & 24.93 & 29.11 \\
\hline & Rate(kbps) & 90.34 & 515.00 & 89.89 & 512.65 & 93.18 & 520.20 & 89.75 & 513.51 \\
\hline & ET(s) & 1.98 & 1.86 & 2.87 & 3.27 & 2.14 & 1.95 & 3.07 & 2.77 \\
\hline \multirow{3}{*}{$\begin{array}{c}\mathrm{JM} \\
\mathrm{RC} \\
Q P^{*}\end{array}$} & Y-PSNR(dB) & 24.96 & 28.93 & 25.08 & 29.07 & 24.88 & 28.84 & 25.02 & 28.98 \\
\hline & Rate(kbps) & 98.24 & 494.75 & 99.81 & 496.15 & 99.85 & 495.73 & 99.30 & 496.02 \\
\hline & ET(s) & 1.93 & 1.89 & 2.78 & 3.32 & 2.13 & 1.98 & 3.09 & 2.91 \\
\hline \multirow{3}{*}{$\begin{array}{c}\text { Proposed } \\
\text { RC } \\
Q P^{*}-10\end{array}$} & Y-PSNR(dB) & 25.06 & 29.19 & 25.17 & 29.26 & 24.99 & 29.13 & 25.13 & 29.21 \\
\hline & Rate(kbps) & 93.22 & 516.94 & 92.31 & 512.48 & 94.96 & 523.92 & 91.52 & 514.32 \\
\hline & ET(s) & 2.07 & 1.99 & 2.79 & 3.25 & 2.17 & 1.90 & 3.01 & 3.07 \\
\hline \multirow{3}{*}{$\begin{array}{c}\mathrm{JM} \\
\mathrm{RC} \\
Q P^{*}-10\end{array}$} & Y-PSNR $(\mathrm{dB})$ & 24.82 & 28.73 & 24.94 & 28.85 & 24.72 & 28.63 & 24.91 & 28.75 \\
\hline & Rate(kbps) & 90.31 & 473.18 & 89.51 & 473.87 & 89.64 & 473.19 & 89.67 & 474.53 \\
\hline & $\mathrm{ET}(\mathrm{s})$ & 2.01 & 1.84 & 2.73 & 3.19 & 2.19 & 1.97 & 2.99 & 2.89 \\
\hline \multirow{3}{*}{$\begin{array}{c}\text { Proposed } \\
\text { RC } \\
Q P^{*}+10\end{array}$} & Y-PSNR(dB) & 24.83 & 29.06 & 24.89 & 29.12 & 24.75 & 29.02 & 24.85 & 29.07 \\
\hline & Rate(kbps) & 94.32 & 522.93 & 93.12 & 515.92 & 97.79 & 524.26 & 94.98 & 520.45 \\
\hline & $\mathrm{ET}(\mathrm{s})$ & 2.13 & 2.01 & 2.85 & 3.16 & 2.27 & 2.04 & 3.04 & 2.90 \\
\hline \multirow{3}{*}{$\begin{array}{c}\mathrm{JM} \\
\mathrm{RC} \\
Q P^{*}+10\end{array}$} & Y-PSNR $(\mathrm{dB})$ & 24.63 & 28.90 & 24.70 & 29.00 & 24.55 & 28.82 & 24.69 & 28.92 \\
\hline & Rate(kbps) & 100.29 & 499.67 & 100.76 & 502.45 & 100.48 & 500.01 & 100.96 & 504.53 \\
\hline & ET(s) & 2.07 & 1.92 & 2.69 & 3.14 & 2.13 & 1.97 & 3.02 & 2.91 \\
\hline
\end{tabular}

\section{APPENDIX A}

Proof OF EQUations (5) AND (6)

The probability density function (pdf) of transform coefficients with i.i.d. zero-mean Laplacian distribution is:

$$
p(x)=\frac{1}{\sqrt{2} \sigma} e^{-\frac{\sqrt{2}|x|}{\sigma}}=\frac{\Lambda}{2} e^{-\Lambda|x|}
$$

where $x$ represents the transformed residual, $\sigma$ is the standard deviation, and $\Lambda=\sqrt{2} / \sigma$ is the Laplace parameter. For the uniform quantizer with quantization step size $Q$ and rounding offset $\gamma Q$, the probability of zero after quantization is

$$
P_{0}=\int_{-(Q-\gamma Q)}^{Q-\gamma Q} p(x) d x=2 \int_{0}^{Q(1-\gamma)} p(x) d x=1-e^{-\Lambda Q(1-\gamma)}
$$


and the probability of transformed residuals inside $n$th quantization interval is

$$
P_{n}=\int_{n Q-\gamma Q}^{(n+1) Q-\gamma Q} p(x) d x=\int_{Q(n-\gamma)}^{Q(n+1-\gamma)} p(x) d x=\frac{1}{2}\left(1-e^{-\Lambda Q}\right) e^{\Lambda Q \gamma} e^{-\Lambda Q n}
$$

According to the entropy definition, therefore, the source rate can be approximated by the entropy of the quantized transformed residuals, derived as

$$
\begin{aligned}
R(\Lambda, Q)=H(\Lambda, Q) & =-P_{0} \log _{2} P_{0}-2 \sum_{n=1}^{\infty} P_{n} \log _{2} P_{n} \\
& =-P_{0} \log _{2} P_{0}+\left(1-P_{0}\right)\left[\frac{\Lambda Q \log _{2} e}{1-e^{-\Lambda Q}}-\log _{2}\left(1-e^{-\Lambda Q}\right)-\Lambda Q \gamma \log _{2} e+1\right]
\end{aligned}
$$

On the other hand, the total quantization distortion is derived by summing up the quantization distortion in each quantization interval as follows

$$
\begin{aligned}
D(\Lambda, Q) & =\int_{-(Q-\gamma Q)}^{Q-\gamma Q} x^{2} p(x) d x+2 \sum_{n=1}^{\infty} \int_{n Q-\gamma Q}^{(n+1) Q-\gamma Q}(x-n Q)^{2} p(x) d x \\
& =\frac{\Lambda Q e^{\gamma \Lambda Q}(2+\Lambda Q-2 \gamma \Lambda Q)+2-2 e^{\Lambda Q}}{\Lambda^{2}\left(1-e^{\Lambda Q}\right)}
\end{aligned}
$$

\section{APPENDIX B}

\section{DERIVATION OF EQUATION (19)}

According to KKT conditions, the first-order necessary conditions of optimality for problem Eq. (16) require the primal solutions $\left(\lambda^{*}, Q^{*}\right)$ and dual solutions $\left(\mu^{*}, \eta^{*}\right)$ such that

$$
\begin{aligned}
& \frac{\partial L\left(\lambda^{*}, Q^{*}, \mu^{*}, \eta^{*}\right)}{\partial \lambda}=\frac{\partial D\left(\lambda^{*}, Q^{*}\right)}{\partial \lambda}+\mu^{*} \cdot \frac{\partial R\left(\lambda^{*}, Q^{*}\right)}{\partial \lambda}+\eta^{*} \cdot \frac{\partial d\left(\lambda^{*}, Q^{*}\right)}{\partial \lambda}=0 \\
& \frac{\partial L\left(\lambda^{*}, Q^{*}, \mu^{*}, \eta^{*}\right)}{\partial Q}=\frac{\partial D\left(\lambda^{*}, Q^{*}\right)}{\partial Q}+\mu^{*} \cdot \frac{\partial R\left(\lambda^{*}, Q^{*}\right)}{\partial Q}+\eta^{*} \cdot \frac{\partial d\left(\lambda^{*}, Q^{*}\right)}{\partial Q}=0 \\
& d\left(\lambda^{*}, Q^{*}\right)=\frac{N\left(2 \lambda^{*}+1\right)^{2} \theta \cdot \alpha\left(Q^{*}\right) \cdot c_{0}}{\sqrt[3]{k^{-1} P_{\max }}} \\
& \mu^{*} \cdot\left[R\left(\lambda^{*}, Q^{*}\right)-R_{\max }\right]=0 \\
& \eta^{*} \cdot\left[d\left(\lambda^{*}, Q^{*}\right)-d_{\max }\right]=0
\end{aligned}
$$

Let us first consider using the Newton's method to solve the set of nonlinear equations (34a) 
- (34e), when $\mu^{*}$ and $\eta^{*}$ are not equal to 0 , as follows

$$
\begin{gathered}
\frac{\partial L\left(\lambda^{k}, Q^{k}, \mu^{k}, \eta^{k}\right)}{\partial \lambda}+\frac{\partial^{2} L\left(\lambda^{k}, Q^{k}, \mu^{k}, \eta^{k}\right)}{\partial \lambda^{2}}\left(\lambda^{k+1}-\lambda^{k}\right)+\frac{\partial^{2} L\left(\lambda^{k}, Q^{k}, \mu^{k}, \eta^{k}\right)}{\partial \lambda \partial Q}\left(Q^{k+1}-Q^{k}\right) \\
+\frac{\partial R\left(\lambda^{k}, Q^{k}\right)}{\partial \lambda}\left(\mu^{k+1}-\mu^{k}\right)+\frac{\partial d\left(\lambda^{k}, Q^{k}\right)}{\partial \lambda}\left(\eta^{k+1}-\eta^{k}\right)=0 \\
\frac{\partial L\left(\lambda^{k}, Q^{k}, \mu^{k}, \eta^{k}\right)}{\partial Q}+\frac{\partial^{2} L\left(\lambda^{k}, Q^{k}, \mu^{k}, \eta^{k}\right)}{\partial Q \partial \lambda}\left(\lambda^{k+1}-\lambda^{k}\right)+\frac{\partial^{2} L\left(\lambda^{k}, Q^{k}, \mu^{k}, \eta^{k}\right)}{\partial Q^{2}}\left(Q^{k+1}-Q^{k}\right) \\
+\frac{\partial R\left(\lambda^{k}, Q^{k}\right)}{\partial Q}\left(\mu^{k+1}-\mu^{k}\right)+\frac{\partial d\left(\lambda^{k}, Q^{k}\right)}{\partial Q}\left(\eta^{k+1}-\eta^{k}\right)=0 \\
R\left(\lambda^{k}, Q^{k}\right)-R_{\max }+\frac{\partial R\left(\lambda^{k}, Q^{k}\right)}{\partial \lambda}\left(\lambda^{k+1}-\lambda^{k}\right)+\frac{\partial R\left(\lambda^{k}, Q^{k}\right)}{\partial Q}\left(Q^{k+1}-Q^{k}\right)=0 \\
d\left(\lambda^{k}, Q^{k}\right)-d_{\max }+\frac{\partial d\left(\lambda^{k}, Q^{k}\right)}{\partial \lambda}\left(\lambda^{k+1}-\lambda^{k}\right)+\frac{\partial d\left(\lambda^{k}, Q^{k}\right)}{\partial Q}\left(Q^{k+1}-Q^{k}\right)=0
\end{gathered}
$$

where a new iterate $\left(\lambda^{k+1}, Q^{k+1}, \mu^{k+1}, \eta^{k+1}\right)$ is obtained by solving the first-order approximation, given a current iterate $\left(\lambda^{k}, Q^{k}, \mu^{k}, \eta^{k}\right)$. For all the defined functions, the derivative operators $\nabla$ and $\nabla^{2}$, respectively, are used to refer to the first-order gradient and the second-order Hessian matrix with respect to primal variables $\lambda$ and $Q$. Furthermore, denoting $\delta^{k}=\left(\delta_{\lambda}^{k}, \delta_{Q}^{k}\right)^{T}=\left(\lambda^{k+1}-\right.$ $\left.\lambda^{k}, Q^{k+1}-Q^{k}\right)^{T}$ as the vector representing the update directions of primal variables, the set of equations can be written as

$$
\left(\begin{array}{c}
\nabla L\left(\lambda^{k}, Q^{k}, \mu^{k}, \eta^{k}\right) \\
R\left(\lambda^{k}, Q^{k}\right)-R_{\max } \\
d\left(\lambda^{k}, Q^{k}\right)-d_{\max }
\end{array}\right)+\left(\begin{array}{ccc}
\nabla^{2} L\left(\lambda^{k}, Q^{k}, \mu^{k}, \eta^{k}\right) & \nabla R\left(\lambda^{k}, Q^{k}\right) & \nabla d\left(\lambda^{k}, Q^{k}\right) \\
\nabla R\left(\lambda^{k}, Q^{k}\right) & 0 & 0 \\
\nabla d\left(\lambda^{k}, Q^{k}\right) & 0 & 0
\end{array}\right)\left(\begin{array}{c}
\delta^{k} \\
\mu^{k+1}-\mu^{k} \\
\eta^{k+1}-\eta^{k}
\end{array}\right)=\mathbf{0}
$$

Eq. (36) can be further written as

$$
\left(\begin{array}{ccc}
\nabla^{2} L\left(\lambda^{k}, Q^{k}, \mu^{k}, \eta^{k}\right) & \nabla R\left(\lambda^{k}, Q^{k}\right) & \nabla d\left(\lambda^{k}, Q^{k}\right) \\
\nabla R\left(\lambda^{k}, Q^{k}\right) & 0 & 0 \\
\nabla d\left(\lambda^{k}, Q^{k}\right) & 0 & 0
\end{array}\right)\left(\begin{array}{c}
\delta^{k} \\
\mu^{k+1} \\
\eta^{k+1}
\end{array}\right)=\left(\begin{array}{c}
-\nabla D\left(\lambda^{k}, Q^{k}\right) \\
R_{\max }-R\left(\lambda^{k}, Q^{k}\right) \\
d_{\max }-d\left(\lambda^{k}, Q^{k}\right)
\end{array}\right)
$$

At the $k$ th iteration, the linear system in Eq. (37) can be solved for $\delta^{k}, \mu^{k+1}$, and $\eta^{k+1}$, and then set $\left(\lambda^{k+1}, Q^{k+1}\right)^{T}=\left(\lambda^{k}, Q^{k}\right)^{T}+\delta^{k}$ for the next iteration. As the process is repeated until $\delta^{k}=\mathbf{0}$, we shall have found the stationary point to problem Eq. (16). Equivalently, given an iterate $\left(\lambda^{k}, Q^{k}, \mu^{k}, \eta^{k}\right)$, a new iterate $\left(\lambda^{k+1}, Q^{k+1}, \mu^{k+1}, \eta^{k+1}\right)$ can also be obtained by solving a 
quadratic programming (QP) minimization subproblem given by Eq. (19). It can be noted that the optimum $\delta^{k}$ to Eq. (19), together with the optimal Lagrange multipliers $\mu^{k+1}$ and $\eta^{k+1}$ associated with the linearized constraints, is a stationary point for Eq. (19) and satisfies Eqs. (35a)-(35d) when $\mu^{k+1}$ and $\eta^{k+1}$ are grater than 0 . In order to iteratively find an optimal solution for Eq. (16), hence, Eq. (19) can be employed in lieu of the linear system (37).

\section{REFERENCES}

[1] Z. He, Y. Liang, L. Chen, I. Ahmad, and D. Wu, "Power-rate-distortion analysis for wireless video communication under energy constraints," IEEE Transactions on Circuits and Systems for Video Technology, vol. 15, no. 5, pp. 645-658, May 2005.

[2] Sandvine, "Global internet phenomena report," 2012. [Online]. Available: http://www.sandvine.com/downloads/documents/Phenomena_1H_2012/Sandvine_Global_Internet_Phenomena_Report_1H_2012.pdf

[3] “Global mobile data traffic forecast update, 2012-2017," Cisco Visual Networking Index (VNI) Forecast, 2013. [Online]. Available: http://www.cisco.com/en/US/solutions/collateral/ns341/ns525/ns537/ ns705/ns827/white_paper_c11-520862.pdf

[4] X. Li, N. Oertel, A. Hutter, and A. Kaup, "Laplace distribution based Lagrangian rate distortion optimization for hybrid video coding," IEEE Transactions on Circuits and Systems for Video Technology, vol. 19, no. 2, pp. 193-205, Feb. 2009.

[5] C. Y. Hsu, A. Ortega, and M. Khansari, "Rate control for robust video transmission over burst-error wireless channels," IEEE Journal on Selected Areas in Communications, vol. 17, no. 5, pp. 756-773, May 1999.

[6] Q. Chen, "Image and video processing for denoising, coding, and content protection," Ph.D. dissertation, University of Florida, 2011.

[7] S. Soltani, K. Misra, and H. Radha, "Delay constraint error control protocol for real-time video communication," IEEE Transactions on Multimedia, vol. 11, no. 4, pp. 742-751, Jun. 2009.

[8] Q. Chen and D. Wu, "Delay-rate-distortion model for rea-time video communication," submitted to IEEE Transactions on Circuits and Systems for Video Technology for review, 2013.

[9] S. Khan, Y. Peng, E. Steinbach, M. Sgroi, and W. Kellerer, "Application-driven cross-layer optimization for video streaming over wireless networks," IEEE Communications Magazine, vol. 44, no. 1, pp. 122-130, Jan. 2006.

[10] Z. Li, F. Pan, K. Lim, G. Feng, X. Lin, and S. Rahardja, "Adaptive basic unit layer rate control for jvt," in JVT-G012-rl, 7th Meeting, Pattaya II, Thailand, vol. 14, Mar. 2003.

[11] D. Kwon, M. Shen, and C. Kuo, "Rate control for H. 264 video with enhanced rate and distortion models," IEEE Transactions on Circuits and Systems for Video Technology, vol. 17, no. 5, pp. 517-529, May 2007.

[12] H. Wang and S. Kwong, "Rate-distortion optimization of rate control for H. 264 with adaptive initial quantization parameter determination," IEEE Transactions on Circuits and Systems for Video Technology, vol. 18, no. 1, pp. 140-144, Jan. 2008.

[13] B. Yan and K. Sun, "Joint complexity estimation of i-frame and p-frame for h. 264/avc rate control," IEEE Transactions on Circuits and Systems for Video Technology, vol. 22, no. 5, pp. 790-798, May 2012.

[14] S. Ma, W. Gao, and Y. Lu, "Rate-distortion analysis for H. 264/AVC video coding and its application to rate control," IEEE Transactions on Circuits and Systems for Video Technology, vol. 15, no. 12, pp. 1533-1544, Dec. 2005.

[15] I. E. G. Richardson, "H.264/MPEG-4 Part 10: Transform and Quantization," 2003. [Online]. Available: http://www.vcodex.com 
[16] Z. Chen and D. Wu, "Rate-distortion optimized cross-layer rate control in wireless video communication," IEEE Transactions on Circuits and Systems for Video Technology, vol. 22, no. 3, pp. 352-365, Mar. 2012.

[17] A. Aminlou, Z. NajafiHaghi, M. Namaki-Shoushtari, and M. R. Hashemi, "Rate-distortion-complexity optimization for vlsi implementation of integer motion estimation in h. 264/avc encoder," in Proceedings of IEEE International Conference on Multimedia and Expo. IEEE, 2011, pp. 1-6.

[18] J. Vanne, M. Viitanen, T. Hamalainen, and A. Hallpuro, "Comparative rate-distortion-complexity analysis of hevc and avc video codecs," IEEE Transactions on Circuits and Systems for Video Technology, vol. 22, no. 12, pp. 1885-1898, Dec. 2012.

[19] G. Corrêa, P. Assuncao, L. A. da Silva Cruz, and L. Agostini, "Adaptive coding tree for complexity control of high efficiency video encoders," in Picture Coding Symposium. IEEE, 2012, pp. 425-428.

[20] H. M. Hang and J. J. Chen, "Source model for transform video coder and its application. I. Fundamental theory," IEEE Transactions on Circuits and Systems for Video Technology, vol. 7, no. 2, pp. 287-298, Apr. 1997.

[21] G. S. Yovanof and S. Liu, "Statistical analysis of the DCT coefficients and their quantization error," in Conference Record of the 30th Asilomar Conference on Signals, Systems and Computers, vol. 1, Nov. 1996, pp. 601-605.

[22] N. Kamaci, Y. Altunbasak, and R. M. Mersereau, "Frame bit allocation for the H.264/AVC video coder via Cauchy-densitybased rate and distortion models," IEEE Transactions on Circuits and Systems for Video Technology, vol. 15, no. 8, pp. 994-1006, Aug. 2005.

[23] T. Wiegand, G. J. Sullivan, G. Bjontegaard, and A. Luthra, "Overview of the H.264/AVC video coding standard," IEEE Transactions on Circuits and Systems for Video Technology, vol. 13, no. 7, pp. 560-576, Jul. 2003.

[24] K. Sühring, "H.264/AVC reference software JM18.2," $\quad$ Feb. 2012. [Online]. Available: http://iphome.hhi.de/suehring/tml/download/

[25] R. Min, T. Furrer, and A. Chandrakasan, "Dynamic voltage scaling techniques for distributed microsensor networks," in Proceedings of IEEE Computer Society Workshop on VLSI, 2000, pp. 43-46.

[26] J. R. Lorch, A. J. Smith et al., "Improving dynamic voltage scaling algorithms with PACE," ACM SIGMETRICS Performance Evaluation Review, vol. 29, no. 1, pp. 50-61, Jun. 2001.

[27] T. D. Burd and R. W. Brodersen, "Processor design for portable systems," The Journal of VLSI Signal Processing, vol. 13, no. 2, pp. 203-221, 1996.

[28] E. Lam and J. Goodman, "A mathematical analysis of the DCT coefficient distributions for images," IEEE Transactions on Image Processing, vol. 9, no. 10, pp. 1661-1666, Oct. 2000.

[29] S. P. Boyd and L. Vandenberghe, Convex optimization. Cambridge university press, 2004.

[30] H. Everett, "Generalized Lagrange multiplier method for solving problems of optimum allocation of resources," Operations research, vol. 11, no. 3, pp. 399-417, May-Jun. 1963.

[31] X. Li, N. Oertel, A. Hutter, and A. Kaup, "Advanced Lagrange multiplier selection for hybrid video coding," in Proceedings of IEEE International Conference on Multimedia and Expo. IEEE, 2007, pp. 364-367.

[32] Y. Shoham and A. Gersho, "Efficient bit allocation for an arbitrary set of quantizers," IEEE Transactions on Acoustics, Speech and Signal Processing, vol. 36, no. 9, pp. 1445-1453, Sep. 1988.

[33] A. Ortega and K. Ramchandran, "Rate-distortion methods for image and video compression," IEEE Signal Processing Magazine, vol. 15, no. 6, pp. 23-50, Nov. 1998.

[34] D. Bertsekas, Nonlinear programming. Athena Scientific, 1999. 
[35] B. Chachuat, Nonlinear and dynamic optimization: From Theory to Practice. Switzerland: Automatic Control Laboratory, EPFL, 2007.

[36] J. Nocedal and S. J. Wright, Numerical optimization. Springer, 2006.

[37] T. Cover and J. Thomas, Elements of information theory. Wiley-interscience, 2006. 